Inference for Local Distributions at High Sampling Frequencies: A Bootstrap Approach

\author{
Ulrich Hounyo and Rasmus T. Varneskov
}

CREATES Research Paper 2018-16 


\title{
Inference for Local Distributions at High Sampling Frequencies: A Bootstrap Approach*
}

\author{
Ulrich Hounyo $^{\dagger} \quad$ Rasmus T. Varneskov ${ }^{\ddagger}$
}

April 25, 2018

\begin{abstract}
We study inference for the local innovations of Itô semimartingales. Specifically, we construct a resampling procedure for the empirical CDF of high-frequency innovations that have been standardized using a nonparametric estimate of its stochastic scale (volatility) and truncated to rid the effect of "large" jumps. Our locally dependent wild bootstrap (LDWB) accommodate issues related to the stochastic scale and jumps as well as account for a special block-wise dependence structure induced by sampling errors. We show that the LDWB replicates first and second-order limit theory from the usual empirical process and the stochastic scale estimate, respectively, as well as an asymptotic bias. Moreover, we design the LDWB sufficiently general to establish asymptotic equivalence between it and and a nonparametric local block bootstrap, also introduced here, up to second-order distribution theory. Finally, we introduce LDWB-aided Kolmogorov-Smirnov tests for local Gaussianity as well as local von-Mises statistics, with and without bootstrap inference, and establish their asymptotic validity using the second-order distribution theory. The finite sample performance of CLT and LDWB-aided local Gaussianity tests are assessed in a simulation study as well as two empirical applications. Whereas the CLT test is oversized, even in large samples, the size of the LDWB tests are accurate, even in small samples. The empirical analysis verifies this pattern, in addition to providing new insights about the distributional properties of equity indices, commodities, exchange rates and popular macro finance variables.
\end{abstract}

Keywords: Bootstrap inference, High-frequency data, Itô semimartingales, Kolmogorov-Smirnov test, Stable processes, von-Mises statistics.

JEL classification: C12, C14, C15, G1

\footnotetext{
${ }^{*}$ We wish to thank Viktor Todorov for helpful discussions. Financial support from CREATES, Center for Research in Econometric Analysis of Time Series (DNRF78), funded by the Danish National Research Foundation, is gratefully acknowledged.

${ }^{\dagger}$ Department of Economics, University at Albany - State University of New York, Albany, NY 12222; CREATES, Aarhus, Denmark; e-mail: khounyo@albany.edu.

${ }^{\ddagger}$ Department of Finance, Copenhagen Business School, 2000 Frederiksberg, Denmark; CREATES, Aarhus, Denmark; Multi Assets at Nordea Asset Management, Copenhagen, Denmark; e-mail: rtv.fi@cbs.dk.
} 


\section{Introduction}

Itô semimartingales comprise an important class of continuous time processes that are widely used in finance and economics, among others, to describe the evolution of financial asset prices, exchange rates, interest rates, commodities, asset return volatility, derivatives prices, volume of trades, innovations in aggregate consumption as well as network traffic. This broad class of processes include jump-diffusions as the, unequivocally, most commonly adopted subclass of models across a variety of applications, see e.g., Andersen \& Benzoni (2012) and many references therein. This subclass, for specificity, characterizes the innovations to the process of interest as a stochastic differential equation of the form,

$$
d Z_{t}=\alpha_{t} d t+\sigma_{t-} d W_{t}+d Y_{t}
$$

where the drift $\alpha_{t}$ and volatility $\sigma_{t}$ are processes with càdlàg paths, $W_{t}$ is a standard Brownian motion and $Y_{t}$ is an Itô semimartingale of the pure-jump type (formal assumptions are given below). The model in (1) allows $Z_{t}$ to follow a drift, subject to innovations of the mixed Gaussian type $\left(\sigma_{t}\right.$ being the stochastic mixing scale) and display larger, and more infrequent, jumps. Moreover, by allowing for correlation between the increments $d \sigma_{t}$ and $d Z_{t}$ to be correlated, the model can capture leverage or volatility feedback effects, working through either continuous or discontinuous (or jump) channels. Importantly, despite allowing for general continuous and discontinuous sample paths as well as correlation between the various components of the model, the specification (1) is consistent with no arbitrage in financial markets, e.g., Back (1991) and Delbaen \& Schachermayer (1994).

Despite the fact that the behavior of (1) may be very complex at longer time horizons, its structure simplifies considerably at high sampling frequencies. To see this, suppose that $t$ is restricted to the interval $[0,1]$, and we consider its (infill) asymptotic behavior for some shrinking time interval from $t$ to $t+s h$ with $h \rightarrow 0$, then the Brownian motion will dominate the drift and the jump components, provided that the stochastic scale, $\sigma_{t}$, is non-vanishing. That is, for fixed $0 \leq t<s \leq 1$,

$$
h^{-1 / 2} \frac{Z_{t+s h}-Z_{t}}{\sigma_{t}} \stackrel{d}{\rightarrow} W_{t+s}^{\prime}-W_{s}^{\prime}, \quad \text { as } \quad h \rightarrow 0
$$

where $W_{t}^{\prime}$ is a standard Brownian motion (again, technical details are given below). Hence, (2) highlights that the model in (1) is locally mean-zero and mixed Gaussian with stochastic scale, illustrating that the model is capable of generating fat-tail returns, even at high-sampling frequencies, through the mixture-of-distributions effect, e.g., Clark (1973). Moreover, it makes strong predictions about the local distributional properties of the standardized innovations; namely Gaussianity. This assumption is fundamental, not only when describing the dynamics of asset and state variables (such as those listed above) as well as when pricing derivatives, but also for many multivariate problems where correlations are critical to the analysis, e.g., portfolio allocation. The intricate relation (2) facilitates testing of this fundamental assumption. This feature is important, especially since local Gaussianity rules out another class of Itô semimartingales of the pure-jump type, which has recently been demonstated, 
using different nonparametric techniques, to provide an accurate description of the local distributional properties of various assets at high sampling frequencies, see e.g., Todorov \& Tauchen (2011b), Jing, Kong \& Liu (2012), Andersen, Bondarenko, Todorov \& Tauchen (2015), and Hounyo \& Varneskov (2017). Such pure-jump semimartingales may be characterized similarly to (1) with, however, the Brownian increments replaced by a Lévy jump process of infinite variation. Under general conditions, the latter can be shown to be locally equivalent to a stable process, $S_{t}$, with activity (or tail) index $1<\beta<2$, e.g., Todorov \& Tauchen (2012). As is illustrated by Figure 1, a stable distribution with activity index lower than the Gaussian boundary case $\beta=2$ is characterized by having fatter, possibly asymmetric, tails and larger excess kurtosis than a comparable Gaussian distribution. Hence, the selection between modeling paradigms - jump-diffusions and pure-jump semimartingales - amounts to testing whether the local (and standardized) increments at high sampling frequencies are better described by a Gaussian or stable distribution with $1<\beta<2$. If these increments, indeed, are stable, this alters the way we need to study a plethora of economic phenomena, exemplified by the parametric, using stable distribution settings, analyses of asset returns, e.g., Mandelbrot (1961, 1963), Fama (1963), Fama \& Roll (1968) and, more recently, Carr, Geman, Madan \& Yor (2002) and Kelly \& Jiang (2014); option pricing, e.g., Carr \& Wu (2003, 2004) and Andersen, Fusari, Todorov \& Varneskov (2018); volatility modeling, e.g., Barndorff-Nielsen \& Shephard (2001), Carr, Geman, Madan \& Yor (2003) and Todorov, Tauchen \& Grynkiv (2014); network traffic, e.g., Mikosch, Resnik, Rootzen \& Stegeman (2002); and electricity prices, e.g., Klüppelberg, Meyer-Brandi \& Schmidt (2010). Moreover, Aït-Sahalia \& Jacod (2009) and Todorov \& Tauchen (2011a), among others, show that the magnitude of $\beta$ is essential for the estimation of, and inference on, risk measures such as power variation.

In this paper, we seek to draw inference on the local distributional properties of $Z_{t}$. This is particularly challenging in the present setting vis-a-vis (1), since $Z_{t}$, in addition to exhibiting local distributional properties that may either be Gaussian or stable, can have a stochastic drift, $\alpha_{t}$, a stochastic scale, $\sigma_{t}$, well as "residual" jumps, $Y_{t}$. Specifically, we consider bootstrap inference based on the empirical CDF statistic by Todorov \& Tauchen (2014), which, asymptotically, recover the distribution of the locally leading term, $W_{t}$ or $S_{t}$, by nonparametrically standardizing and truncating high-frequency increments of $Z_{t}$ in its construction. While the standardization and truncation alleviate estimation errors generated by $\sigma_{t}$ and $Y_{t}$, respectively, to recover information about the locally leading term, they show that a bias-correction is generally needed and develop asymptotic central limit theory for the first-order estimation error - its empirical process - as well as the (higher-order) estimation sampling error that arises from having replaced the unobservable scale, $\sigma_{t}$, with a nonparametric estimate. This second-order distribution theory is utilized in designing a Kolmogorov-Smirnov (KS) test for local Gaussianity. However, despite having strong theoretical appeal, the KS test performs unstably, and often unsatisfactory, in their finite sample Monte Carlo study, rejecting (wrongfully) either $10.3 \%$ or $32.8 \%$ of the times when the nominal size is $1 \%$ or $5 \%$, respectively, thus highlighting the need for improved inference and testing procedures. The bootstrap represents a natural alternative to inference based on central limit theory. However, in addition to facing the same challenges as 
Todorov \& Tauchen (2014), the bootstrap procedures, we seek to develop, will not only need to replicate the first-order limit theory, but also account for the bias and the second-order distribution. This combination of issues is unprecedented in the bootstrap literature.

The first contribution of the paper is to provide a locally dependent wild bootstrap (LDWB) procedure that enables inference on the local distribution of the leading term of $Z_{t}$. To this end, following to the discussion above, we accommodate issues related to the stochastic scale and residual jumps as well as design the bootstrap to account for a special block-wise dependence structure created by the sampling errors that arise when replacing $\sigma_{t}$ with a nonparametric estimate. By accounting for such features, we show that the LDWB is not only asymptotically valid for the first-order distributional properties, the empirical process limit, but also for the second-order distribution and it accommodates the bias. Second, we show that our LDWB framework is general enough to nest a nonparametric local block bootstrap (NLBB), also developed in this paper, thereby establishing asymptotic equivalence between two separate bootstrap paradigms up to a second-order distribution, in a general semimartingale setting. Third, we utilize the bootstrap in designing new Kolmogorov-Smirnov tests for local Gaussianity and establishes their asymptotic properties. Fourth, we design von-Mises statistics based on nonparametrically standardized and truncated high-frequency returns, provide a LDWB procedure for such and establish their asymptotic properties. Both are new to high-frequency financial econometrics and both rely on the second-order distribution theory for the LDWB.

The theoretical contributions of this paper represent advances for two different literatures; bootstrap inference for empirical processes and high-frequency econometric inference and hypothesis testing. First, our bootstrap is related to the dependent wild bootstrap procedures in Shao (2010) and Doukhan, Lang, Leucht \& Neumann (2015), who consider inference on the time series mean of a stationary dependent process and its empirical process, respectively, as well as the block bootstraps in, among others, Bickel \& Freedman (1981), Bühlmann (1994), and Naik-Nimbalkar \& Rajarshi (1994), who consider inference for empirical processes in either i.i.d. or stationary and dependent settings. In particular, and relative to previous bootstraps, the LDWB accommodate non-stationarities in the increments of the observed process through $\sigma_{t}$ and $Y_{t}$, a special block-wise dependence structure, arising from nonparametric standardization errors, and, finally, it asymptotically replicates first and second-order distribution theory. Moreover, we specify the external random variables in the design sufficiently general to establish asymptotic equivalence of the LDWB and NLBB, up to second-order distribution theory. Such results are hitherto not available in the bootstrap literature, even under simplifying assumptions for $Z_{t}$. In relation to the high-frequency financial econometrics literature, we provide new (bootstrap) inference techniques for local distributions in infill asymptotic settings, introduce local von-Mises statistics to the literature (with and without the bootstrap), as well as provide new nonparametric bootstrap-aided tests for local Gaussianity. The contributions closest to ours are Todorov \& Tauchen (2014), as explained above, and the bootstraps for power variations in Gonçalves \& Meddahi (2009), Hounyo \& Varneskov (2017), Hounyo (2018), and Dovonon, Gonçalves, Hounyo \& Meddahi (2018), who consider either local Gaussian or local stable settings. It is important 
to note, however, that direct adaptations of their bootstrap designs will result in inference procedures that looses all dependence on the original data; see Remark 3 and Appendix B below. ${ }^{1}$

In addition to the theoretical contributions, we examine the finite sample properties of KolmogorovSmirnov tests for local Gaussianity based on either the central limit theory (CLT) in Todorov \& Tauchen (2014), the LDWB or the NLBB. Consistent with Todorov \& Tauchen (2014), we find severe size distortions for the CLT-based test, even in large samples. In contrast, the LDWB-aided test enjoys accurate size, even in small samples, as well as good power properties. The NLBB performs similarly to the LDWB, albeit with slightly worse size properties, showing the benefits of our general bootstrap framework. To illustrate the usefulness of the test, we consider two empirical application. First, we test for local Gaussianity in high-frequency futures data on three different asset classes; equity indices, foreign exchange rates and commodities. Interestingly, we find the high-frequency innovations to equity indices and commodities are well-described as Gaussian. In contrast, we strongly reject local Gaussianity for the exchange rate series, which, on the other hand, are better described as locally stable with tail index in the 1.80 to 1.90 range. Moreover, we verify the size results from the simulation study; the CLT rejects uniformly more often than the LDWB-aided test for equity indices and commodities. Second, we demonstrate that the bootstrap procedures are not only applicable to high-frequency data, but may be used more generally as a nonparametric test for local Gaussianity that is robust to heteroskedasticity (or stochastic volatility). ${ }^{2}$ Specifically, we test the distributional properties of four series that are widely used in the macro finance literature, namely the VIX, TIPS, default spread and the term spread. Interestingly, we find strikingly different conclusions from the CLT and LDWB tests. Whereas the former rejects local Gaussianity for all series, the LDWB test only rejects for the VIX and the term spread. Since the latter is consistent with visual evidence, and the critical values vis-a-vis test statistics show borderline rejections for the CLT test and the TIPS and default spread series, we attribute the different results, again, to the finite sample size differences between the two testing procedures, with the LDWB test being accurate.

The paper proceeds as follows. Section 2 introduces the semimartingale framework, the statistics of interest and reviews some critical results. Section 3 introduces the locally dependent wild bootstrap procedure and establishes its asymptotic properties as well as its equivalence to the nonparametric local block bootstrap. Section 4 provides new bootstrap-aided Kolmogorov-Smirnov tests for local Gaus-

\footnotetext{
${ }^{1}$ Our paper is also related to Andersen, Bollerslev \& Dobrev (2007), who consider testing for Gaussianity using daily, or other sufficiently sparse returns, that are standard by realized measures constructed from high-frequency data. Using a sequential procedure, they accommodate jumps and stochastic volatility in their design. However, their testing framework is based on a long time span of "standardized" returns over fixed time spans as well as high-frequency observations, whereas we exclusive rely on the latter. Hence, our time interval is generally shrinking and we utilize local spot volatility measure. Both are crucial for the feasible limit theory and facilitates testing without a long time span of data. Finally, and importantly, we carry out testing using bootstrap procedures.

${ }^{2}$ This represents an alternative to Gaussianity tests based on parametric methods, which are either carried out implicitly when specifying fully parametric heavy-tailed GARCH or stochastic volatility models for, among others, asset return dynamics or explicitly when using such parametric specifications for the stochastic volatility part of asset returns only, in combination with GMM-based tests for the standardized innovations, e.g., Bontemps \& Meddahi (2005). These frameworks both rely on long span time series and asymptotics as well as parametric specifications, whereas our fully nonparametric test relies on infill asymptotics and high-frequency data.
} 
sianity and local von-Mises statistics. Section 5 contains the simulation study, and Section 6 provides the empirical analysis. Finally, Section 7 concludes. Appendices A-C have additional assumptions, theory, proofs, technical results and implementation details.

\section{A General Semimartingale Framework}

This section introduces a general class of semimartingales, the formal assumptions for the theoretical analysis as well as provides examples of such processes in applied work. Moreover, we define the empirical statistics of interest for the bootstrap analysis in the remainder of the paper.

\subsection{Setup and Assumptions}

Suppose the process $Z$ is defined on a filtered probability space, $\left(\Omega, \mathcal{F},\left(\mathcal{F}_{t}\right), \mathbb{P}\right)$, where the information filtration $\left(\mathcal{F}_{t}\right) \subseteq \mathcal{F}$ is an increasing family of $\sigma$-fields satisfying $\mathbb{P}$-completeness and right continuity. Specifically, assume that $Z$ obeys a semimartingale process that generalizes (1) and has the following dynamics

$$
d Z_{t}=\alpha_{t} d t+\sigma_{t-} d S_{t}+d Y_{t}, \quad 0 \leq t \leq 1,
$$

where $\alpha_{t}$ and $\sigma_{t}$ are $\left(\mathcal{F}_{t}\right)$-adapted processes with càdlàg paths, $Y_{t}$ is a pure-jump process of finite activity, and $S_{t}$ is a stable process with stability index $1<\beta \leq 2$, whose (log-)characteristic function is defined as

$$
\ln \mathbb{E}\left[e^{\mathrm{i} u S_{t}}\right]=-t|c u|^{\beta}(1-\mathrm{i} \gamma \operatorname{sign}(u) \tan (\pi \beta / 2)),
$$

where $\gamma \in[-1,1]$ controls its skewness. We have depicted the density of $S_{t}$ for various choices the activity index $\beta$ and skewness $\gamma$ in Figure 1, illustrating how the two affect the skewness, kurtosis and, in particular, the tails of the density. Note that for $\beta=2$ and $c=1 / 2$, the semimartingale process in (3) reduces to the jump-diffusion model in (1). When $1<\beta<2$, on the other hand, $Z_{t}$ is a pure-jump semimartingale of infinite variation for which the innovations to $S_{t}$ still dominate the drift and "residual" jump process, $Y_{t}$, at fine time scales. That is, under the regularity conditions to be outlined below, we have $h^{-1 / \beta}\left(Z_{t+s h}-Z_{t}\right) / \sigma_{t} \stackrel{d}{\rightarrow} S_{t+s}^{\prime}-S_{t}^{\prime}$ as $h \rightarrow 0$ with convergence holding under the Skorokhod topology on the space of càdlàg functions, where $S_{t}^{\prime}$ is a Lévy process with a distribution identical to the one implied by (4). Yet, despite similar scaling properties, the fine scale behavior generated by (4) allows for much richer dynamics relative to a standard Gaussian.

Before proceeding to the assumptions, let $\mathbb{R}_{+}=\{x \in \mathbb{R}: x \geq 0\}$ and $(E, \mathcal{E})$ denote an auxiliary measurable space on the original filtered probability space $\left(\Omega, \mathcal{F},\left(\mathcal{F}_{t}\right), \mathbb{P}\right)$. Moreover, going forward, we write $S_{t}=W_{t}$ when $\beta=2$ and $c=1 / 2$ to emphasize that the model is a jump-diffusion.

Assumption 1. $Z_{t}$ satisfies (3) with the following conditions on its components:

(a) The process $Y_{t}$ obeys

$$
Y_{t}=\int_{0}^{t} \int_{E} \delta^{Y}(s, x) \mu(d s, d x)
$$


where $\mu(d s, d x)$ is a Poisson measure on the space $\mathbb{R}_{+} \times E$, which is characterized by the Lévy measure $\nu(d x)$, and $\delta^{Y}(t, x)$ is some predictable function on $\Omega \times \mathbb{R}_{+} \times E$.

(b) $\left|\sigma_{t}\right|^{-1}$ and $\left|\sigma_{t-}\right|^{-1}$ are strictly positive.

(c) $\sigma_{t}$ is a semimartingale process of the form

$$
\sigma_{t}=\sigma_{0}+\int_{0}^{t} \tilde{\alpha}_{u} d u+\int_{0}^{t} \tilde{\sigma}_{u} d S_{u}+\int_{0}^{t} \tilde{\sigma}_{u}^{\prime} d W_{u}^{\prime}+\int_{0}^{t} \int_{E} \delta^{\sigma}(s, x) \mu(d s, d x),
$$

where $W_{t}^{\prime}$ is a standard Brownian motion independent $S_{t}$, irrespective of $\beta$; the triplet $\tilde{\alpha}_{t}, \tilde{\sigma}_{t}$ and $\tilde{\sigma}_{t}^{\prime}$ are processes with càdlàg paths; and $\delta^{\sigma}(t, x)$ is some predictable function on $\Omega \times \mathbb{R}_{+} \times E$. Moreover, $\tilde{\sigma}_{t}$ and $\tilde{\sigma}_{t}^{\prime}$ are both Itô semimartingales with càdlàg paths and whose jumps being integrals of some predictable functions, $\delta^{\tilde{\sigma}}(t, x)$ and $\delta^{\tilde{\sigma}^{\prime}}(t, x)$, with respect to $\mu(d s, d x)$.

(d) There exists a sequence of stopping times $T_{p}$ on the space $E$, increasing to infinity. Moreover, for each $p, \phi_{p}(x)$ is a non-negative function satisfying $\nu\left(x: \phi_{p}(x) \neq 0\right)<\infty$ such that, for $t \leq T_{p}$,

$$
\left|\delta^{Y}(t, x)\right| \wedge 1+\left|\delta^{\sigma}(t, x)\right| \wedge 1+\left|\delta^{\tilde{\sigma}}(t, x)\right| \wedge 1+\left|\delta^{\tilde{\sigma}^{\prime}}(t, x)\right| \wedge 1 \leq \phi_{p}(x) .
$$

Assumption 1 deserves a few comments. First, the regularity conditions are similar to those imposed by Todorov \& Tauchen (2014, Assumption B). The only two differences are that we refrain from imposing $S_{t}=W_{t}$ here, but will rather make this restriction later, and we allow increments of $S_{t}$ to enter $\sigma_{t}$ in $1(\mathrm{c})$. The main reason for these minor departures is that we wish to state a set of unified conditions under which all subsequent asymptotic results hold, in conjunction with restrictions on $\beta$. Todorov \& Tauchen (2014) uses two separate sets of regularity conditions for their consistency and central limit theory (CLT) analysis. Since we are mainly concerned with inference using bootstrap methods, we will invoke the stronger of those assumptions from the outset. ${ }^{3}$

Second, the conditions in Assumption 1 are very mild. The Itô semimartingale condition on the stochastic scale $\sigma_{t}$ is satisfied in most applications. Moreover, there are no restrictions on the dependence between the residual jumps, $Y_{t}$, and the triplet $\left(S_{t}, \alpha_{t}, \sigma_{t}\right)^{\prime}$. This implies that $Z_{t}$ not necessarily inherits the tail properties of $S_{t}$ at all frequencies and may be driven by a tempered stable process, which can display tail behavior that is very different from that of a stable process.

Third, as examples of jump-diffusion models are plentiful in the literature (see the introduction for references), we end this subsection by providing examples of models that obeys a subclass of the models in (3) with $1<\beta<2$, and which have been successfully applied to describe processes in economics and finance, thus providing powerful alternatives to models with local Gaussianity.

Example 1. Barndorff-Nielsen 83 Shephard (2001) introduce a non-Gaussian OU process for volatility, and Todorov et al. (2014) considers an exponential version of the model, thus accommodating a broader

\footnotetext{
${ }^{3}$ Note that the conditions in Assumption 1 are very similar to those in related work, e.g., Aït-Sahalia \& Jacod (2010), Todorov \& Tauchen (2011a), Hounyo \& Varneskov (2017) as well as references therein.
} 
class of leading jump processes. For illustration, let $\mu$ and $\kappa$ be positive constants, then the exponential version may be written as $\ln \sigma_{t}=\mu+V_{t}$ with $d V_{t}=-\kappa+d L_{t}$ where the driving Lévy process, $L_{t}$, behaves locally (as $h \rightarrow 0$ ) like a stable process with characteristic function (4).

Example 2. Let $S_{t}=S_{t}(\beta, \gamma)$ be a $\beta$-stable random variable with skewness $\gamma$. Moreover, let $r$ and $q$ be the risk-free and dividend rate, respectively, and let $\mu$ be a convexity adjustment, then the log stable option pricing model by Carr $\&$ Wu (2003) is defined as $d Z_{t}=(r-q+\mu) d t+\sigma d S_{t}$.

Example 3. The framework in (3) and (4) accommodate a general class of time changed stochastic processes. Specifically, we can write $Z_{t}=X_{z_{t}}$ where $X_{t}$ is a Lévy process and $z_{t}$ is an increasing process with càdlàg paths. In such a setting, Monroe (1978) shows that all semimartingale processes may be written as a time-changed Brownian motion, and Sato (1999) that Lévy processes subordinated by a positive Lévy process yields new Lévy processes. Wu (2008) give several practical examples of such processes, and Clark (1973) and Ané $\mathcal{E}$ Geman (2000), among others, use the time-change framework to jointly model the number of trades, transaction times and asset returns.

\subsection{Empirical Statistics of Interest}

First, let $Z_{t}$ be observed at an equidistant time grid $t_{i} \in[0,1]$, for $i=0, \ldots, n$, and write the highfrequency increments as $\Delta_{i}^{n} Z=Z_{t_{i}}-Z_{t_{i-1}} \cdot{ }^{4}$ Next, divide the fixed time interval into blocks, each of which containing $k_{n}$ increments with $k_{n} \rightarrow \infty$ and $k_{n} / n \rightarrow 0$. For each block, we compute an estimate of the spot variation $\sigma_{t}^{2}$ by means of the local bipower variation statistic,

$$
\widehat{V}_{n, j}=\frac{\pi}{2} \frac{n}{k_{n}-1} \sum_{i=(j-1) k_{n}+2}^{j k_{n}}\left|\Delta_{i-1}^{n} Z\right|\left|\Delta_{i}^{n} Z\right|, \quad j=1, \ldots,\left\lfloor n / k_{n}\right\rfloor .
$$

Despite $\widehat{V}_{n, j}$ being consistent for $\sigma_{t}^{2}$, we will need to use a modified estimator to scale the highfrequency increments and forming the empirical CDF. Specifically, as we need independence between the $i$ th increment $\Delta_{i}^{n} Z$ in the numerator and the denominator, we will exclude said increment as

$$
\widehat{V}_{n, j}(i)=\left\{\begin{array}{l}
\frac{k_{n}-1}{k_{n}-3} \widehat{V}_{n, j}-\frac{\pi}{2} \frac{n}{k_{n}-3}\left|\Delta_{i}^{n} Z\right|\left|\Delta_{i+1}^{n} Z\right| \quad \text { for } \quad i=(j-1) k_{n}+1 \\
\frac{k_{n}-1}{k_{n}-3} \widehat{V}_{n, j}-\frac{\pi}{2} \frac{n}{k_{n}-3}\left(\left|\Delta_{i-1}^{n} Z\right|\left|\Delta_{i}^{n} Z\right|+\left|\Delta_{i}^{n} Z\right|\left|\Delta_{i+1}^{n} Z\right|\right) \\
\quad \text { for } \quad i=(j-1) k_{n}+2, \ldots, j k_{n}-1 ; \\
\frac{k_{n}-1}{k_{n}-3} \widehat{V}_{n, j}-\frac{\pi}{2} \frac{n}{k_{n}-3}\left|\Delta_{i-1}^{n} Z\right|\left|\Delta_{i}^{n} Z\right|, \quad \text { for } \quad i=j k_{n} .
\end{array}\right.
$$

Now, to form the empirical CDF and devise its feasible CLT, Todorov \& Tauchen (2014) selects only the first $m_{n}$ increments on each block and require $1>m_{n} / k_{n} \rightarrow 0$ as $n \rightarrow \infty$. Intuitively, this is to ensure that the estimation errors from $\widehat{V}_{n, j}(i)$, both its finite sample bias and variance, vanish

\footnotetext{
${ }^{4}$ The definitions of the high-frequency statistics, including notation, follow Todorov \& Tauchen (2014) closely.
} 
sufficiently fast upon averaging relative to the contribution of each block to the empirical CDF. This implies that the total number of increments used for estimation is given by

$$
N_{n}(\alpha, \varpi)=\sum_{j=1}^{\left\lfloor n / k_{n}\right\rfloor} \sum_{i=(j-1) k_{n}+1}^{(j-1) k_{n}+m_{n}} 1\left\{\frac{\sqrt{n}\left|\Delta_{i}^{n} Z\right|}{\sqrt{\widehat{V}_{n, j}}} \leq \alpha n^{1 / 2-\varpi}\right\}
$$

where $\alpha>0$ an $\varpi \in(0,1 / 2)$, and that the empirical CDF is formed as,

$$
\widehat{F}_{n}(\tau)=\frac{1}{N_{n}(\alpha, \varpi)} \sum_{j=1}^{\left\lfloor n / k_{n}\right\rfloor} \sum_{i=(j-1) k_{n}+1}^{(j-1) k_{n}+m_{n}} 1\left\{\frac{\sqrt{n} \Delta_{i}^{n} Z}{\sqrt{\widehat{V}_{n, j}(i)}} \leq \tau\right\} 1\left\{\frac{\sqrt{n}\left|\Delta_{i}^{n} Z\right|}{\sqrt{\widehat{V}_{n, j}}} \leq \alpha n^{1 / 2-\varpi}\right\}
$$

In addition to standardizing the increments by the stochastic scale, the empirical CDF in (8) truncates the increments of $Z_{t}$ to reduce the impact of larger jumps on $\widehat{F}_{n}(\tau)$. While the latter is strictly not needed to obtain consistency and CLT for the latter, the truncation serves to reduce the higher-order bias in $\widehat{F}_{n}(\tau)$ due to jumps. Additionally, note that $N_{n}(\alpha, \varpi) /\left(\left\lfloor n / k_{n}\right\rfloor m_{n}\right) \stackrel{\mathbb{P}}{\rightarrow} 1$. Finally, before stating the asymptotic results due to Todorov \& Tauchen (2014), the following assumption collects rate conditions on the tuning parameters determining the block sizes, $k_{n}$ and $m_{n}$.

Assumption 2. $m_{n}$ and $k_{n}$ satisfy either of the following two conditions as $n \rightarrow \infty$,

(a) $k_{n} \asymp n^{q}$, for some $q \in(0,1)$ and $m_{n} \rightarrow \infty$;

(b) $k_{n} \asymp n^{q}$, for some $q \in(0,1 / 2)$ and $m_{n} / k_{n} \rightarrow 0$ such that $\left(n m_{n}\right) / k_{n}^{3} \rightarrow \lambda \geq 0$.

Lemma 1. If Assumptions 1 and 2(a) hold, then, uniformly in $\tau$ over compact subsets of $\mathbb{R}$,

$$
\widehat{F}_{n}(\tau) \stackrel{\mathbb{P}}{\rightarrow} F_{\beta}(\tau)
$$

where $F_{\beta}(\tau)$ is the CDF of $\sqrt{2 / \pi} \frac{S_{1}}{E\left(S_{1}\right)}$ and $S_{1}$ is the value of the $\beta$-stable process $S_{t}$ at $t=1$. In particular, $F_{2}(\tau)$ equals the CDF of a standard Gaussian random variable $\Phi(\tau)$.

Lemma 2. If Assumptions 1 and 2(b) hold, and let $S_{t}=W_{t}$, i.e., $Z_{t}$ be a jump-diffusion, then, locally uniformly in $\tau$ over compact subsets of $\mathbb{R}$,

$$
\widehat{F}_{n}(\tau)-\Phi(\tau)=\widehat{H}_{n, 1}(\tau)+\widehat{H}_{n, 2}(\tau)+H_{3}(\tau) / k_{n}+o_{p}\left(1 / k_{n}\right)
$$

where $\sqrt{\left\lfloor n / k_{n}\right\rfloor m_{n}}\left(\widehat{H}_{n, 1}(\tau), \sqrt{k_{n} / m_{n}} \widehat{H}_{n, 2}(\tau)\right) \stackrel{d}{\rightarrow}\left(H_{1}(\tau), H_{2}(\tau)\right)$ with $H_{1}(\tau)$ and $H_{2}(\tau)$ being two mean-zero independent Gaussian processes with covariance functions,

$$
\begin{aligned}
& \operatorname{Cov}\left[H_{1}\left(\tau_{1}\right), H_{1}\left(\tau_{2}\right)\right]=\Phi\left(\tau_{1} \wedge \tau_{2}\right)-\Phi\left(\tau_{1}\right) \Phi\left(\tau_{2}\right) \\
& \operatorname{Cov}\left[H_{2}\left(\tau_{1}\right), H_{2}\left(\tau_{2}\right)\right]=\left(\frac{\tau_{1} \Phi^{\prime}\left(\tau_{1}\right)}{2} \frac{\tau_{2} \Phi^{\prime}\left(\tau_{2}\right)}{2}\right)\left(\left(\frac{\pi}{2}\right)^{2}+\pi-3\right),
\end{aligned}
$$


for $\tau_{1}, \tau_{2} \in \mathbb{R}$. Finally,

$$
H_{3}(\tau)=\frac{\tau^{2} \Phi^{\prime \prime}(\tau)-\tau \Phi^{\prime}(\tau)}{8}\left(\left(\frac{\pi}{2}\right)^{2}+\pi-3\right)
$$

Lemma 1 shows that the CDF of standardized increments $\Delta_{i}^{n} Z$ may be estimated consistently, as long as their fine scale behavior belongs to the class stable processes described by (4). Lemma 2 improves this result for the jump-diffusion model, showing that a CLT holds with a rate of convergence that may arbitrarily close to $\sqrt{n}$, depending on $m_{n}$ and $k_{n}$. The limiting distribution, however, are affected by the nonparametric standardization of the increments. For specificity, whereas $H_{1}(\tau)$ is well-known from Donsker's theorem for empirical processes, e.g., van der Vaart (1998), the additional components $H_{2}(\tau)$ and $H_{3}(\tau)$ are lower-order estimation errors and an asymptotic bias, respectively, induced by use of the estimate $\widehat{V}_{n, j}$ rather than the latent $\sigma_{t}$. Importantly, all components of the limit depends only on $\tau$, not on $\sigma_{t}$, meaning that this result is amenable to feasible inference.

By utilizing their CLT result in Lemma 2, Todorov \& Tauchen (2014) design a Kolmogorov-Smirnovtype test for local Gaussianity of $Z_{t}$, i.e., $\mathcal{H}_{0}: S_{t}=W_{t}$, as

$$
\widehat{\mathrm{KS}}_{n}(\mathcal{A})=\sup _{\tau \in \mathcal{A}} \sqrt{N_{n}(\alpha, \varpi)}\left|\widehat{F}_{n}(\tau)-\Phi(\tau)\right|
$$

where $\mathcal{A} \subset \mathbb{R} \backslash 0$ denotes a finite union of compact sets with positive Lebesgue measure. The critical region of the test is $C_{n}(\theta, \mathcal{A})=\left\{\widehat{\mathrm{KS}}_{n}(\mathcal{A})>q_{n}(\theta, \mathcal{A})\right\}$, where $\theta \in(0,1)$, and $q_{n}(\theta, \mathcal{A})$ is the $(1-\theta)$ th quantile of

$$
\sup _{\tau \in \mathcal{A}}\left|H_{1}(\tau)+\sqrt{\frac{m_{n}}{k_{n}}} H_{2}(\tau)+\sqrt{\frac{m_{n}}{k_{n}}} \frac{\sqrt{n}}{k_{n}} H_{3}(\tau)\right| .
$$

The test for local Gaussianity, $\widehat{\mathrm{KS}}_{n}(\mathcal{A})$, similarly to the empirical CDF, contain two additional terms compared with standard Kolmogorov-Smirnov distribution testing, $H_{2}(\tau)$ and $H_{3}(\tau)$, arising from the use of nonparametric, and noisy, estimates of the stochastic scale, $\sigma_{t}$, when standardizing the increments. Whereas the second term is of strictly lower order by $m_{n} / k_{n} \rightarrow 0$ as $n \rightarrow \infty$, the bias term has first-order impact since $\sqrt{\left(m_{n} n\right) / k_{n}^{3}} \rightarrow \sqrt{\lambda} \geq 0$. Hence, not only does the limit theory in Lemma 2 aid the correction of systematic testing errors by accounting for $H_{3}(\tau)$, the explicit utilization of higher-order asymptotic theory through $H_{2}(\tau)$ may generate improved testing properties in finite samples, where the ratio $m_{n} / k_{n}$ can be non-trivial. However, when gauging the size results for their test in Todorov \& Tauchen $(2014$, Table 1$), \widehat{\mathrm{KS}}_{n}(\mathcal{A})$ is seen to be very sensitive to block size $k_{n}$ and may display large distortions, e.g., rejecting either $10.3 \%$ or $32.8 \%$ of the times when the nominal size is $1 \%$ or $5 \%$, respectively, highlighting the need for improved testing procedures.

In what follows, we will study inference for the empirical CDF as well as testing for local Gaussianity using bootstrap methods to restore the size properties of such tests. However, Lemma 2 shows that this is particularly challenging in the present setting since such bootstrap procedures need not only to replicate the first-order distribution theory, reflected by $H_{1}(\tau)$, asymptotically, but also to account for the asymptotic bias, $H_{3}(\tau)$, as well as to replicate the higher-order limit theory, $H_{2}(\tau)$. 
Remark 1. The central limit theory is provided on compact sets of $\tau, \mathcal{A} \subset \mathbb{R} \backslash 0$, since the error in the estimation of the CDF for $\tau \rightarrow \pm \infty$ due to large jumps is affected by truncation. As a result, the bootstrap methods, we develop below, will similarly apply to the set $\mathcal{A}$.

Remark 2. Market microstructure noise is a concern when sampling the observations at very high frequencies. For example, more frequently than every minute or every 15 ticks, see, e.g., Hansen ES Lunde (2006) and Bandi $\&$ Russell (2008). Suppose, in this case, that the observed increments decompose $\Delta_{i}^{n} \tilde{Z}=\Delta_{i}^{n} Z+\Delta_{i}^{n} \mathcal{N}$ where $\mathcal{N}_{t_{i}}, i=1, \ldots, n$ are i.i.d. random variables, defined on a product extension of the original probability space and are independent of the filtration $\mathcal{F}$. Then, Todorov 8 Tauchen (2014) shows that the empirical CDF converges to the CDF of standardized noise increments, which differs from $\Phi(\tau)$, thus providing a different violation of local Gaussianity.

\section{Bootstrapping the Empirical CDF at High Frequency}

In this section, we introduce a new and general resampling procedure - the locally dependent wild bootstrap - to draw inference on the empirical CDF in (8) as well as for testing whether $Z_{t}$ is better described by a jump-diffusion model (1) against the alternative in (3) with $1<\beta<2$, that is, to test local Gaussianity against distributions with fatter tails and, possibly, skewness. Specifically, the bootstrap resamples centered, standardized and dependent observations using a (possibly, dependent) external random variable. We establish the asymptotic properties of the procedure as well as discuss the similarities and differences between related bootstrap procedures in the classical time series and empirical process literature, in particular, a nonparametric local block bootstrap.

\subsection{Bootstrap Notation}

As is standard in the bootstrap literature, $\mathbb{P}^{*}, \mathbb{E}^{*}$ and $\mathbb{V}^{*}$ denote the probability measure, expected value and variance, respectively, induced by the resampling and is, thus, conditional on a realization of the original time series. For any bootstrap statistic $Z_{n}^{*} \equiv Z_{n}^{*}(\cdot, \omega)$ and any (measurable) set $A$, we write $\mathbb{P}^{*}\left(Z_{n}^{*} \in A\right)=\mathbb{P}^{*}\left(Z_{n}^{*}(\cdot, \omega) \in A\right)=\operatorname{Pr}\left(Z_{n}^{*}(\cdot, \omega) \in A \mid \mathcal{X}_{n}\right)$, where $\mathcal{X}_{n}$ denotes the observed sample. Moreover, we say $Z_{n}^{*} \stackrel{\mathbb{P}^{*}}{\rightarrow} 0$ in probability- $\mathbb{P}\left(\right.$ or $Z_{n}^{*}=o_{p}^{*}(1)$ in probability- $\mathbb{P}$ ) if for any $\varepsilon>0$, $\delta>0, \lim _{n \rightarrow \infty} \mathbb{P}\left[\mathbb{P}^{*}\left(\left|Z_{n}^{*}\right|>\delta\right)>\varepsilon\right]=0$. Similarly, $Z_{n}^{*}=O_{p}^{*}(1)$ in probability- $\mathbb{P}$ if for all $\varepsilon>0$ there exists an $M_{\varepsilon}<\infty$ such that $\lim _{n \rightarrow \infty} \mathbb{P}\left[\mathbb{P}^{*}\left(\left|Z_{n}^{*}\right|>M_{\varepsilon}\right)>\varepsilon\right]=0$. Finally, for a sequence of random variables (or vectors) $Z_{n}^{*}$, a definition of weak convergence (convergence in distribution) in probability$\mathbb{P}$ is needed. Hence, we write $Z_{n}^{*} \stackrel{d^{*}}{\rightarrow} Z$ as $n \rightarrow \infty$, if, conditional on the sample, $Z_{n}^{*}$ converges weakly to $Z$ under $\mathbb{P}^{*}$, for all samples contained in a set with probability- $\mathbb{P}$ approaching one.

\subsection{The Local Dependent Wild Bootstrap for the Empirical CDF}

The framework in Section 2 presents several challenges that are unprecedented in the bootstrap literature, e.g., the combination of the general class processes in (3), the infill asymptotic setting and the 
need for replication of higher-order central limit theory. To overcome such challenges, we design a new and general resampling procedure - the locally dependent wild bootstrap (LDWB) - which is inspired by the dependent wild bootstraps (DWBs) in Shao (2010) and Doukhan et al. (2015), but, as will be detailed below, differs in subtle, yet important ways, to remain valid in the present setting.

First, let us define

$$
X_{(j-1) k_{n}+i} \equiv 1\left\{\frac{\sqrt{n} \Delta_{(j-1) k_{n}+i}^{n} Z}{\sqrt{\widehat{V}_{n, j}\left((j-1) k_{n}+i\right)}} \leq \tau\right\} 1\left\{\frac{\sqrt{n}\left|\Delta_{(j-1) k_{n}+i}^{n} Z\right|}{\sqrt{\widehat{V}_{n, j}}} \leq \alpha n^{1 / 2-\varpi}\right\}
$$

for $j=1, \ldots,\left\lfloor n / k_{n}\right\rfloor$ and $i=1, \ldots, m_{n}$, and use this to write

$$
\widetilde{F}_{n}(\tau)=\frac{N_{n}(\alpha, \varpi)}{\left\lfloor n / k_{n}\right\rfloor m_{n}} \widehat{F}_{n}(\tau)=\frac{1}{\left\lfloor n / k_{n}\right\rfloor m_{n}} \sum_{j=1}^{\left\lfloor n / k_{n}\right\rfloor} \sum_{i=1}^{m_{n}} X_{(j-1) k_{n}+i}
$$

The random increments and empirical CDF in (12) and (13), respectively, illustrate the differences between the present bootstrap setting and the corresponding in Doukhan et al. (2015), who also consider DWB inference for empirical processes. In our case, the problem is more challenging due to the distributional properties of (3) may differ at coarse and fine time scales, depending on $S_{t}$ and $Y_{t}$, which necessitates an infill asymptotic approach to estimation and the identification of the locally dominant stochastic component, $S_{t}$. Moreover, the process (3) is allowed to have a stochastic scale (volatility, if Gaussian), time-varying drift and display jumps, in contrast with the stationarity requirement for the data generating process in Doukhan et al. (2015, Assumption A1). Third, the nonparametric standardization of the increments in (12) creates a nonlinear $m_{n}$-dependence within blocks (that is, across $i$ ), which impact the bootstrap design as well as its asymptotic theory. In particular, and as highlighted by Lemma 2, our local DWB need not only to replicate the first-order asymptotic theory, it needs to account for an asymptotic bias as well as to replicate the higher-order limit theory, generated by the nonparametric estimates of the stochastic scale used for the standardization.

Specifically, our LDWB resamples the centered, locally (and nonparametrically) standardized and truncated increments in (12) as follows

$$
X_{(j-1) k_{n}+i}^{*}=\widetilde{F}_{n}(\tau)+\frac{N_{n}(\alpha, \varpi)}{\left\lfloor n / k_{n}\right\rfloor m_{n}}\left(X_{(j-1) k_{n}+i}-\widetilde{F}_{n}(\tau)\right) v_{(j-1) k_{n}+i}^{*}
$$

where $v_{i}^{*}, i=1, \ldots, n$, is a sequence of external random variables subject to mild regularity conditions, which are formalized below. The bootstrap variables in (14) may, then, be utilized in designing a new inference procedure for the empirical CDF at high (i.e., infill) sampling frequencies as

$$
\widehat{F}_{W, n}^{*}(\tau)=\frac{1}{N_{n}(\alpha, \varpi)} \sum_{j=1}^{\left\lfloor n / k_{n}\right\rfloor} \sum_{i=1}^{m_{n}} X_{(j-1) k_{n}+i}^{*}
$$




$$
=\widehat{F}_{n}(\tau)+\frac{1}{\left\lfloor n / k_{n}\right\rfloor m_{n}} \sum_{j=1}^{\left\lfloor n / k_{n}\right\rfloor} \sum_{i=1}^{m_{n}}\left(X_{(j-1) k_{n}+i}-\widetilde{F}_{n}(\tau)\right) v_{(j-1) k_{n}+i}^{*} .
$$

The LWDB decomposes into the empirical CDF, $\widehat{F}_{n}(\tau)$, capturing the "mean" of the bootstrap statistic and an "innovation" aimed at capturing its distribution. The asymptotic properties of (15), however, depend crucially on $v_{i}^{*}$, and we impose the following, general, conditions:

Assumption DWB. The sequence of random variables $v_{i}^{*}, i=1, \ldots, n$, is stationary, independent of the observed sample path $\mathcal{X}_{n}$ and satisfies the follow regularity conditions:

(a) $\mathbb{E}\left[v_{i}^{*}\right]=0, \mathbb{V}\left[v_{i}^{*}\right] \rightarrow 1$ and $\mathbb{E}\left[\left|v_{i}^{*}\right|^{4}\right]<\infty$.

(b) $\operatorname{Cov}\left(v_{i}^{*}, v_{j}^{*}\right) \rightarrow C_{i, j}$ for $i \neq j$ where $C_{j, i} \geq 0$ is a nonrandom constant.

(c) $v_{i}^{*}$ is $b_{n}$-dependent with $\sum_{r=1}^{\left\lfloor n / k_{n}\right\rfloor m_{n}} \operatorname{Cov}\left(v_{1}^{*}, v_{r}^{*}\right)=O\left(b_{n}\right)$ for some $b_{n} / m_{n} \rightarrow \rho \geq 0$ as $n \rightarrow \infty$.

Together with the decompositions in (14) and (15), Assumption DWB highlight some important features of the LDWB. First, the centering of the external random variable in the resampling implies that $\mathbb{E}^{*}\left[\widehat{F}_{W, n}^{*}(\tau)\right]=\widehat{F}_{n}(\tau)$, that is, the LDWB implicitly corrects for the asymptotic bias in the empirical CDF. Second, time series dependence in $X_{(j-1) k_{n}+i}$ plays a different role in our setting compared with in Shao (2010) and Doukhan et al. (2015). Whereas they seek to replicate a lead-lag covariance structure of the observations, needing a condition of the form $\operatorname{Cov}\left(v_{s}^{*}, v_{r}^{*}\right) \rightarrow 1$ as $n \rightarrow \infty$, dependence in the present setting is created by the unwarranted estimation errors in $\widehat{V}_{n, j}$, which are perfectly dependent within a given block $j=1, \ldots,\left\lfloor n / k_{n}\right\rfloor$, but independent across blocks, generating a tradeoff between the rate of convergence, the asymptotic bias and the impact from the higher-order distribution. Third, it is important to note that the leading impact from these estimation errors are generated by Brownian increments (see Lemmas A.1-A.2 in the appendix), which have trivial lead-lag dependence. Hence, we accommodate $\operatorname{Cov}\left(v_{i}^{*}, v_{j}^{*}\right) \rightarrow C_{i, j}$ where $C_{j, i} \geq 0$ is a generic nonrandom constant as well as dependence that does not match the blocks, i.e., the case $b_{n} / m_{n} \rightarrow 0$. In fact, in the infill asymptotic limit, since the stochastic scale in (3) is approximately constant over a block, we allow $v_{i}^{*} \sim$ i.i.d. $(0,1)$, subject to a bounded fourth moment. Fourth, we need to impose an upper bound the dependence, $b_{n} / m_{n} \rightarrow \varrho>0$, since $b_{n}$ controls the asymptotic order of the "noise" coming from the nonparametric estimator $\widehat{V}_{n, j}$ in the resampling, similarly to $m_{n}$ in the original statistic. Fifth, despite it strictly not being needed to replicate the distribution theory in Lemma 2, it may be preferable designing the bootstrap with $\operatorname{Cov}\left(v_{i}^{*}, v_{j}^{*}\right) \rightarrow 1$ and $b_{n} \asymp m_{n}$, since, e.g., Lemma A.2(b) in the appendix shows that this would aid the replication of higher-order covariance from the secondorder distribution term, $H_{2}(\tau)$. Finally, whereas Doukhan et al. (2015) require $v_{i}^{*}$ to be Gaussian, we avoid parameterizing its distribution. This is critical for the (asymptotic) analysis of the similarities between the LDWB and a local nonparametric block bootstrap in the next section.

These features of the resampling, in conjunction with the standardization and truncation of the increments in (12) allow us to accommodate the array of additional challenges in the present setting 
and replicate the asymptotic inference of the bias-corrected empirical process,

$$
\widehat{\mathcal{G}}_{n}(\tau)=\sqrt{N_{n}(\alpha, \varpi)}\left(\widehat{F}_{n}(\tau)-\Phi(\tau)-H_{3}(\tau) / k_{n}\right), \quad \text { i.e. } \quad \mathcal{G}_{n}(\tau) \equiv H_{1}(\tau)+\sqrt{\frac{m_{n}}{k_{n}}} H_{2}(\tau),
$$

using the LDWB in (15), hence, up to second order. This formalized in the following theorem.

Theorem 1. Suppose the conditions of Lemma 2 as well as Assumption DWB hold. Then, locally uniformly in $\tau$ over compact subsets of $\mathbb{R}$, it follows that

(a) $\widehat{\mathcal{F}}_{W, n}^{*}(\tau) \equiv \sqrt{N_{n}(\alpha, \varpi)}\left(\widehat{F}_{W, n}^{*}(\tau)-\widehat{F}_{n}(\tau)\right) \stackrel{d^{*}}{\rightarrow} \mathcal{G}_{n}(\tau)$, in probability-P

(b) $\sup _{x \in \mathbb{R}}\left|\mathbb{P}^{*}\left(\widehat{\mathcal{F}}_{W, n}^{*}(\tau) \leq x\right)-\mathbb{P}\left(\widehat{\mathcal{G}}_{n}(\tau) \leq x\right)\right| \stackrel{\mathbb{P}}{\rightarrow} 0$.

Theorem 1 demonstrates that our LWDB for nonparametrically standardized and truncated increments replicates the asymptotic distribution of the bias-corrected empirical CDF statistic up to second order. Not only is this feature achieved in the general setting (3), allowing for time-varying drift, stochastic volatility and jumps in the underlying process of interest as well as mild conditions on the external random variables, the central limit theory goes well-beyond the corresponding results for the respective DWBs in Shao (2010) and Doukhan et al. (2015), who provide first-order limits, which, in our setting, is equivalent to establishing $\widehat{\mathcal{F}}_{W, n}^{*}(\tau) \stackrel{d^{*}}{\rightarrow} H_{1}(\tau)$, in probability-P. Similar comments apply to classical results in the bootstrap literature for empirical processes, e.g., for the i.i.d setup in Bickel \& Freedman (1981) as well as for block bootstrap methods applied to stationary and dependent processes in Bühlmann (1994) and Naik-Nimbalkar \& Rajarshi (1994). Hence, both the LDWB procedure as well as its second-order asymptotic theory are new to the bootstrap literature. Furthermore, we formally analyze the similarities block bootstrap methods and the LDWB in the next section. Finally, and as indicated in Section 2.2, the replication of second-order limit theory is very important in the present setting, as it alleviates the inference errors due to the use of a nonparametric spot volatility estimator $\widehat{V}_{n, j}$, converging at a slower rate $n^{1 / 4}$, instead of the latent $\sigma_{t}$.

Remark 3. Assumption DWB accommodates local Gaussian resampling, that is, $v_{i}^{*} \sim N(0,1)$. However, it is important to note that this bootstrap, using (14), is distinct from the local Gaussian bootstrap for power variation statistics in Hounyo (2018), who resamples the increments $\Delta_{i}^{n} Z$ and establishes third-order refinements in a Brownian semimartingale setting. In fact, in Appendix B, we show that the use of this "standard" local Gaussian resampling scheme looses all dependence on the original data in the present setting and, thus, no longer provide bootstrap inference for the empirical CDF, but rather can be interpreted as a simulation based inference procedure.

Remark 4. In addition to local Gaussian resampling, several locally dependent processes satisfies Assumption DWB. Two examples, following Shao (2010) and Doukhan et al. (2015), see also the bootstraps in Leucht $\&$ Neumann (2013) and Smeekes $\&$ Urbain (2014), are autoregressive (AR) and 
moving average (MA) processes, defined for $i=1, \ldots, n$ as

$$
v_{i}^{*}=e^{-1 / b_{n}} v_{i-1}^{*}+\xi_{i} \quad \text { and } \quad v_{i}^{*}=\varsigma_{i}+\ldots \varsigma_{i-b_{n}+1},
$$

respectively, with $\xi_{i} \sim N\left(0,1-e^{-2 / b_{n}}\right)$ and $\varsigma_{i} \sim N\left(0,1 / b_{n}\right)$ are both i.i.d. The finite sample properties of both resampling procedures are examined in the simulation study.

\subsection{The Local DWB vs The Nonparametric Block Bootstrap}

Whereas DWB procedures are relatively new to the resampling literature, starting with Shao (2010), block bootstrap methods for dependent processes have been actively researched since the seminal contributions by Carlstein (1986), Kunsch (1989) and Liu \& Singh (1992), who study various time series problems, and by Bühlmann (1994) and Naik-Nimbalkar \& Rajarshi (1994), who consider inference for empirical processes. Hence, as a natural alternative to the LDWB, and inspired by the extant literature, we propose a nonparametric local block bootstrap (NLBB). Moreover, we will formally show that our LDWB is general enough to nest the NLBB, thus providing a theoretical link between the two separate strands of the resampling literature, in a general setting.

First, for the design of the NLBB, we, once again, utilize that the original time series, $X_{(j-1) k_{n}+i}$ with $j=1, \ldots,\left\lfloor n / k_{n}\right\rfloor$ and $i=1, \ldots, m_{n}$, has a special nonlinear block-dependence structure across $i$ for a given $j$, generated by the standardization with the nonparametric estimate $\widehat{V}_{n, j}$. To this end, define a sequence of blocks $B_{j}=\left\{X_{(j-1) k_{n}+i} ; i=1, \ldots, m_{n}\right\}$ for $j=1, \ldots,\left\lfloor n / k_{n}\right\rfloor$, then our proposed resampling procedure draws $\left\lfloor n / k_{n}\right\rfloor$ blocks randomly with replacement and patches them together to form a bootstrap series, inspired by, e.g., the non-overlapping block bootstrap in Carlstein (1986). The resampling, thus, preserves the $m_{n}$-dependence within each block as well as the asymptotic independence between blocks. To formalize the discussion, let $\mathcal{I}_{j}, j=1, \ldots,\left\lfloor n / k_{n}\right\rfloor$, be i.i.d random variables distributed uniformly on $\left\{1, \ldots,\left\lfloor n / k_{n}\right\rfloor\right\}$, then we may write

$$
X_{(j-1) k_{n}+i}^{\diamond} \equiv X_{\left(\mathcal{I}_{j}-1\right) k_{n}+i}, \quad i, \ldots, m_{n} \quad \text { and } \quad j=1, \ldots\left\lfloor n / k_{n}\right\rfloor
$$

and use these to define block bootstrap (BB) versions of $\widetilde{F}_{n}(\tau)$ and $\widehat{F}_{n}(\tau)$ as

$$
\widetilde{F}_{B B, n}^{*}(\tau)=\frac{1}{\left\lfloor n / k_{n}\right\rfloor m_{n}} \sum_{j=1}^{\left\lfloor n / k_{n}\right\rfloor} \sum_{i=1}^{m_{n}} X_{(j-1) k_{n}+i}^{\diamond}, \quad \widehat{F}_{B B, n}^{*}(\tau)=\frac{\left\lfloor n / k_{n}\right\rfloor m_{n}}{N_{n}(\alpha, \varpi)} \widetilde{F}_{B B, n}^{*}(\tau),
$$

respectively. Next, let $p_{n}=\left\lfloor n / k_{n}\right\rfloor$ be the number of blocks, then it is important to note that representation (18) may equivalently be written using sequence of multinomial random variables with probability $1 / p_{n}$ and number of trials $p_{n}$, defined as $\zeta_{p_{n}, j}^{\diamond}, j=1, \ldots, p_{n}$. Specifically,

$$
\widetilde{F}_{B B, n}^{*}(\tau)=\frac{1}{\left\lfloor n / k_{n}\right\rfloor m_{n}} \sum_{j=1}^{\left\lfloor n / k_{n}\right\rfloor} \zeta_{p_{n}, j}^{\diamond} \sum_{i=1}^{m_{n}} X_{(j-1) k_{n}+i}
$$


where $\zeta_{p_{n}, j}^{\diamond}$ signify the number of times the $j$ th block, $B_{j}$, has been (re-)drawn randomly from the total set of blocks. By the properties of multinomial random variables, it follows that

$$
\mathbb{E}\left[\zeta_{p_{n}, j}^{\diamond}\right]=1, \quad \mathbb{V}\left[\zeta_{p_{n}, j}^{\diamond}\right]=1-1 / p_{n}, \quad \operatorname{Cov}\left(\zeta_{p_{n}, j}, \zeta_{p_{n}, i}\right)=-1 / p_{n} \quad \text { when } \quad i \neq j,
$$

and, importantly, that $\sum_{j=1}^{p_{n}} \zeta_{p_{n}, j}^{\diamond}=p_{n}$. Now, by utilizing these properties and defining the external random variable $v_{(j-1) k_{n}+i}^{\diamond}=\zeta_{p_{n}, j}^{\diamond}-1$ for $i=1, \ldots, m_{n}$ across blocks $j=1, \ldots,\left\lfloor n / k_{n}\right\rfloor$, we may rewrite the representation (19) using addition and subtraction as

$$
\widetilde{F}_{B B, n}^{*}(\tau)=\widetilde{F}_{n}(\tau)+\frac{1}{\left\lfloor n / k_{n}\right\rfloor m_{n}} \sum_{j=1}^{\left\lfloor n / k_{n}\right\rfloor} \sum_{i=1}^{m_{n}}\left(X_{(j-1) k_{n}+i}-\widetilde{F}_{n}(\tau)\right) v_{(j-1) k_{n}+i}^{\diamond}
$$

thus on the same form as the LWDB in (15). Indeed, the following lemma establishes that the sequence of random variables $v_{(j-1) k_{n}+i}^{\diamond}$ satisfy the regularity conditions imposed in Assumption DWB.

Lemma 3. Define $\mathcal{M}_{n, j}=\left\{(j-1) k_{n}+i ; i=1, \ldots, m_{n}\right\}$ for the blocks $j=1, \ldots\left\lfloor n / k_{n}\right\rfloor$, then the sequence of observations $v_{(j-1) k_{n}+i}^{\diamond}, i=1, \ldots m_{n}$ and $j=1, \ldots,\left\lfloor n / k_{n}\right\rfloor$ satisfy,

(a) $\mathbb{E}\left[v_{i}^{\diamond}\right]=0, \mathbb{V}\left[v_{i}^{\diamond}\right]=1-1 / p_{n}$ and $\mathbb{E}\left[\left|v_{i}^{\diamond}\right|^{4}\right]<\infty$.

(b) $\operatorname{Cov}\left(v_{i}^{\diamond}, v_{g}^{\diamond}\right)=1-1 / p_{n}$ for $i, g \in \mathcal{M}_{n, j}$.

(c) $\operatorname{Cov}\left(v_{i}^{\diamond}, v_{g}^{\diamond}\right)=-1 / p_{n}$ for $i \in \mathcal{M}_{n, j}, g \in \mathcal{M}_{n, j^{\prime}}$ and $j \neq j^{\prime}$.

(d) $v_{i}^{\diamond}$ is $m_{n}$-dependent with $\sum_{i=1}^{\left\lfloor n / k_{n}\right\rfloor m_{n}} \operatorname{Cov}\left(v_{1}^{\diamond}, v_{i}^{\diamond}\right)=o\left(m_{n}\right)$.

Hence, Theorem 1 and Lemma 3 may be combined to show:

Corollary 1. Locally uniformly in $\tau$ over compact subsets of $\mathbb{R}$, it follows that

(a) $\widehat{\mathcal{F}}_{B B, n}^{*}(\tau) \equiv \sqrt{N_{n}(\alpha, \varpi)}\left(\widehat{F}_{B B, n}^{*}(\tau)-\widehat{F}_{n}(\tau)\right) \stackrel{d^{*}}{\rightarrow} \mathcal{G}_{n}(\tau)$, in probability- $\mathbb{P}$,

(b) $\sup _{x \in \mathbb{R}}\left|\mathbb{P}^{*}\left(\widehat{\mathcal{F}}_{B B, n}^{*}(\tau) \leq x\right)-\mathbb{P}\left(\widehat{\mathcal{G}}_{n}(\tau) \leq x\right)\right| \stackrel{\mathbb{P}}{\rightarrow} 0$.

Lemma 3 and Corollary 1 are intriguing, demonstrating that the general class of LDWBs nests the NLBB and, consequently, that the latter also replicates the second-order distribution theory for the empirical CDF. The nesting result is related to prior results on the exchangeability of weighted bootstraps for the empirical process. Specifically, in a setting with i.i.d. observations, Præstgaard \& Wellner (1993) show that the seminal block bootstrap by Efron (1979) is nested within a general class weighted bootstraps for empirical processes that replicates the asymptotic distribution of a Brownian bridge (that is, of $H_{1}(\tau)$ ). Moreover, Shao (2010) establishes that the bias and variance of the DWB for long-run variance estimation are second-order equivalent to those for the tapered block bootstrap of Paparoditis \& Politis $(2001,2002)$, whose properties are generally favorable to those of moving 
block bootstraps, e.g., Kunsch (1989) and Liu \& Singh (1992). Hence, our result provides additional insights into the relation between bootstrap paradigms. First, the LWDB can be interpreted as a generally weighted bootstrap, with Assumption DWB providing sufficient conditions on the weights. Second, the asymptotic equivalence between DWBs and BBs hold for the general class of processes (3), hence not confined to i.i.d. observations as assumed by prior studies, and it holds for both first and second-order central limit theory. Both results significantly generalizes existing discussions in Præstgaard \& Wellner (1993) and Shao (2010). Finally, the second-order replication of the central limit theory generalizes prior first-order results for BBs in Carlstein (1986) as well as for empirical processes in Bühlmann (1994) and Naik-Nimbalkar \& Rajarshi (1994).

Remark 5. The $N L B B$ is designed using non-overlapping blocks, as in Carlstein (1986), due to natural block-dependence of the nonparametrically standardized data. It may be feasible to consider moving blocks as well, if the differential dependence within a (moving) block is accounted for, e.g., by an additional external variable. The design of such a resampling procedure, including its (asymptotic) relation to the $L D W B$, is not straightforward and we leave it for further research.

Remark 6. The representation in $N L B B$ is reminiscent of the blockwise wild bootstrap for spectral testing of white noise against serial dependence in Shao (2011). Specifically, using a similar block structure as above, Shao (2011) proposes to use an external i.i.d. variable with $\mathbb{E}\left[u_{i}^{*}\right]=0, \mathbb{V}\left[u_{i}^{*}\right]=1$ as well as $\mathbb{E}\left[\left|u_{i}^{*}\right|^{4}\right]<\infty$. Hence, by the same arguments provided for the $N L B B$, one can show that the blockwise bootstrap is, similarly, nested within the LDWB class in the present, general, setting.

\section{Testing for Local Gaussianity at High Frequencies}

This section introduces new bootstrap-aided tests for local Gaussianity of (3). First, and similarly to Todorov \& Tauchen (2014), we provide a LDWB Kolmogorov-Smirnov (KS) test. Second, we propose new Cramér-von Mises (CM) statistics for the empirical CDF at high frequencies and provide associated tests for local Gaussianity, based on either the limit theory in Lemma 2 or the LDWB. The introduction of CM-based tests is motivated, in part, by Shapiro \& Wilk (1965), Shapiro, Wilk $\&$ Chen (1968) and Stephens (1974), who show that the former enjoys non-trivial power advantages over KS procedures when testing for Gaussianity in many (albeit, more traditional) settings.

\subsection{Bootstrap Kolmogorov-Smirnov Testing}

In analogy with the KS test in (11), we define a LWDB version of the test statistic and the corresponding critical region of the bootstrap test by

$$
\mathrm{KS}_{n}^{*}(\mathcal{A})=\sup _{\tau \in \mathcal{A}} \sqrt{N_{n}(\alpha, \varpi)}\left|\widehat{F}_{W, n}^{*}(\tau)-\widehat{F}_{n}(\tau)\right|, \quad C_{n}^{*}(\mathcal{A})=\left\{\widehat{\mathrm{KS}}_{n}(\mathcal{A})>q_{n}^{*}(\theta, \mathcal{A})\right\},
$$


respectively, where, again, $\theta \in(0,1), \mathcal{A} \subset \mathbb{R} \backslash 0$ is a finite union of compact sets with positive Lebesgue measure and $q_{n}^{*}(\theta, \mathcal{A})$ is the $(1-\theta)$ th quantile of the LWDB distribution,

$$
\sup _{\tau \in \mathcal{A}}\left|\frac{\sqrt{N_{n}(\alpha, \varpi)}}{\left\lfloor n / k_{n}\right\rfloor m_{n}} \sum_{j=1}^{\left\lfloor n / k_{n}\right\rfloor} \sum_{i=1}^{m_{n}}\left(X_{(j-1) k_{n}+i}-\widetilde{F}_{n}(\tau)\right) v_{(j-1) k_{n}+i}^{*}\right| .
$$

The validity of the LDWB-aided KS test follows directly from Lemma 2 and Theorem 1 :

Theorem 2. Suppose the regularity conditions for Theorem 1 hold. Moreover, define the quantile function $q_{n}^{*}(\theta, \mathcal{A})=\inf \left\{x \in \mathcal{A}: \mathbb{P}^{*}\left(\operatorname{KS}_{n}^{*}(\mathcal{A})>x\right) \geq \theta\right\}$. Then, for any compact subset $\mathcal{A} \subset \mathbb{R} \backslash 0$ with positive Lebesgue measure, it follows that $\mathbb{P}\left(\widehat{\mathrm{KS}}_{n}(\mathcal{A})>q_{n}^{*}(\theta, \mathcal{A})\right) \rightarrow \theta$ as $n \rightarrow \infty$.

\section{2 von Mises Statistics and Testing}

Let $\ell: \mathbb{R}^{2} \rightarrow \mathbb{R}$ denote a measurable function, whose double integral is assumed to exist, then we may write general von-Mises $\left(V\right.$-)statistics for a $\mathrm{CDF}$ statistic $\mathcal{C}=\left\{\widehat{F}_{n}-H_{3} / k_{n}, \Phi\right\}$, that is, either the bias-corrected empirical CDF or its limit under the null hypothesis, as

$$
\mathcal{V}_{\ell}(\mathcal{C}, \mathcal{A})=\int_{\tau_{1} \in \mathcal{A}} \int_{\tau_{2} \in \mathcal{A}} \ell\left(\tau_{1}, \tau_{2}\right) d \mathcal{C}\left(\tau_{1}\right) d \mathcal{C}\left(\tau_{2}\right)
$$

where, unlike standard $V$-statistics, we restrict integration to the compact set $\mathcal{A} \subset \mathbb{R} \backslash 0$, again, to avoid the truncation of big jumps affecting the central limit theory. Now, let us further impose:

Assumption 3. $\ell$ is continuous, bounded and symmetric in its arguments $\ell\left(\tau_{1}, \tau_{2}\right)=\ell\left(\tau_{2}, \tau_{1}\right)$. Moreover, let $\ell, \ell_{\Phi}(\cdot)=\int_{\tau_{2} \in \mathcal{A}} \ell\left(\cdot, \tau_{2}\right) d \Phi\left(\tau_{2}\right)$, and $\ell\left(\tau_{1}, \cdot\right)$ have bounded variation.

Let $h(\tau-)$ denote the limit from the left of a function $h$ at a point $\tau$, then by Lemma 2 and Assumption 3, we may invoke Beutner \& Zähle (2014, Lemmas 3.4 and 3.6) to decompose

$$
\begin{aligned}
& \mathcal{V}_{\ell}\left(\widehat{F}_{n}-H_{3} / k_{n}, \mathcal{A}\right)-\mathcal{V}_{\ell}(\Phi, \mathcal{A})=-2 \int_{\tau_{1} \in \mathcal{A}}\left(\widehat{F}_{n}-\Phi-H_{3} / k_{n}\right)\left(\tau_{1}-\right) d \ell_{\Phi}\left(\tau_{1}\right) \\
& +\int_{\tau_{1} \in \mathcal{A}} \int_{\tau_{2} \in \mathcal{A}}\left(\widehat{F}_{n}-\Phi-H_{3} / k_{n}\right)\left(\tau_{1}-\right)\left(\widehat{F}_{n}-\Phi-H_{3} / k_{n}\right)\left(\tau_{2}-\right) d \ell\left(\tau_{1}, \tau_{2}\right) \equiv \mathcal{V}_{\ell, \mathrm{N}}(\mathcal{A})+\mathcal{V}_{\ell, \mathrm{D}}(\mathcal{A}),
\end{aligned}
$$

whose parts are typically labeled non-degenerate and degenerate $\left(\ell_{\Phi}\left(\tau_{1}\right) \equiv 0\right)$, respectively. Examples of non-degenerate $V$-statistics are Gini's mean difference and CDF-based variance estimation. Notice, however, that under $\mathcal{H}_{0}: S_{t}=W_{t}$, the infill asymptotic limit of standardized increments (2) are standard Gaussian, subject to estimation errors from the nonparametric stochastic scale, or a meanzero stable process with characteristic function (4) under the alternative, making testing of such features less interesting. ${ }^{5}$ Hence, we focus on the degenerate part, $\mathcal{V}_{\ell, \mathrm{D}}(\mathcal{A})$, for which we can construct tests of the local (again, infill asymptotic) distributional properties of the increments $d Z_{t}$.

\footnotetext{
${ }^{5}$ In fact, we cannot recover the drift of (3) in an infill asymptotic setting, see Jacod (2012).
} 
Theorem 3. Suppose the conditions of Lemma 2 and Assumption 3 hold. Then, locally uniformly for indices $\tau_{1}, \tau_{2} \in \mathcal{A}$,

$$
N_{n}(\alpha, \varpi) \mathcal{V}_{\ell, \mathrm{D}}(\mathcal{A}) \stackrel{d}{\rightarrow} \int_{\tau_{1} \in \mathcal{A}} \int_{\tau_{2} \in \mathcal{A}} \mathcal{G}_{n}\left(\tau_{1}\right) \mathcal{G}_{n}\left(\tau_{2}\right) d \ell\left(\tau_{1}, \tau_{2}\right) .
$$

The general result for $V$-statistics in Theorem 3 goes beyond the asymptotic analysis of the empirical CDF in Todorov \& Tauchen (2014) and facilitates general test statistics of the $L_{2}$-type to examine local distributional properties of $d Z_{t}$. In particular, the asymptotic result allows us to introduce a new class of weighted and bias-corrected Cramér-von Mises tests for $\mathcal{H}_{0}$,

$$
\widehat{\mathrm{CM}}_{n}(k, \mathcal{A})=N_{n}(\alpha, \varpi) \int_{\tau \in \mathcal{A}} k(\tau)\left(\widehat{F}_{n}(\tau)-\Phi(\tau)-H_{3}(\tau) / k_{n}\right)^{2} d \Phi(\tau),
$$

for any measurable weight function $k: \mathbb{R} \rightarrow \mathbb{R}_{+}$, nesting the classical Cramér-von Mises and AndersonDarling weights with $k(\tau)=1$ and $k(\tau)=1 /(\Phi(\tau)(1-\Phi(\tau))$, respectively. Now, by applying the result in Theorem 3, $\widehat{\mathrm{CM}}_{n}(k, \mathcal{A}) \stackrel{d}{\rightarrow} \mathrm{CM}_{n}(k, \mathcal{A})$ where $\mathrm{CM}_{n}(k, \mathcal{A})=\int_{\tau \in \mathcal{A}} k(\tau) \mathcal{G}_{n}(\tau)^{2} d \Phi(\tau) .{ }^{6}$ Similarly to the definitions for the KS test, let $Q_{n}(\theta, k, \mathcal{A})$ be the $(1-\theta)$ th quantile of $\operatorname{CM}_{n}(k, \mathcal{A})$ for $\theta \in(0,1)$, then Lemma 2 and Theorem 3 establish validity of the class of CM tests in (23):

Corollary 2. Suppose the regularity conditions for Theorem 3 hold. Moreover, define the quantile function $Q_{n}(\theta, k, \mathcal{A})=\inf \left\{x \in \mathcal{A}: \mathbb{P}\left(\mathrm{CM}_{n}(k, \mathcal{A})>x\right) \geq \theta\right\}$. Then, for any compact subset $\mathcal{A} \subset \mathbb{R} \backslash 0$ with positive Lebesgue measure, it follows that $\mathbb{P}\left(\widehat{\mathrm{CM}}_{n}(k, \mathcal{A})>Q_{n}(\theta, k, \mathcal{A})\right) \rightarrow \theta$ as $n \rightarrow \infty$.

The class of bias-corrected CM tests in (23) differs, as for the KS test in (11), from standard CM testing by, among others, the contributions of the terms $H_{2}(\tau)$ and $H_{3}(\tau)$ arising from the use nonparametric, and noisy, estimates of the stochastic scale when standardizing the increments as well as the truncation of large jumps in the increments, impacting the integration range.

Remark 7. Although not pursued here, and as discussed in Arcones ES Giné (1992) and Beutner EG Zähle (2014), the statistic $\widehat{\mathrm{S}}_{n}(\mathcal{A})=\int_{\tau \in \mathcal{A}}\left(\widehat{F}_{n}(-\tau)-\left(1-\widehat{F}_{n}(\tau)\right)\right)^{2} d \tau$ may be used to test symmetry of the null distribution. If combined with the CM test in $(23), \widehat{\mathrm{S}}_{n}(\mathcal{A})$ will reveal whether the alternative distribution if $\mathcal{H}_{0}$ is rejected, that is, a local stable, has asymmetric tails.

\subsection{Bootstrap von Mises Statistics and Testing}

The asymptotic distribution in Theorem 3 may be analytically intractable for several choices of kernel function, $\ell$, making inference and testing, e.g., using Corollary 2 hard in practice. However, such difficulties may readily be circumvented using the LDWB. Specifically, let

$$
\mathcal{V}_{\ell, \mathrm{D}}^{*}(\mathcal{A})=\int_{\tau_{1} \in \mathcal{A}} \int_{\tau_{2} \in \mathcal{A}}\left(\widehat{F}_{W, n}^{*}-\widehat{F}_{n}\right)\left(\tau_{1}-\right)\left(\widehat{F}_{W, n}^{*}-\widehat{F}_{n}\right)\left(\tau_{2}-\right) d \ell\left(\tau_{1}, \tau_{2}\right),
$$

\footnotetext{
${ }^{6}$ As explained in Beutner \& Zähle (2014, Example 3.13), the CM test can be viewed as $V$-statistic with kernel function defined by, $\ell\left(\tau_{1}, \tau_{2}\right)=\int_{\tau \in \mathcal{A}} k(\tau)\left(1\left\{\tau_{1} \leq \tau<\infty\right\}-\Phi(\tau)-H_{3}(\tau) / k_{n}\right)\left(1\left\{\tau_{2} \leq \tau<\infty\right\}-\Phi(\tau)-H_{3}(\tau) / k_{n}\right) d \Phi(\tau)$.
} 
be general bootstrapped $V$-statistics, and

$$
\mathrm{CM}_{n}^{*}(k, \mathcal{A})=N_{n}(\alpha, \varpi) \int_{\tau \in \mathcal{A}} k(\tau)\left(\widehat{F}_{W, n}^{*}(\tau)-\widehat{F}_{n}(\tau)\right)^{2} d \Phi(\tau)
$$

the corresponding bootstrap CM test. Moreover, let $Q_{n}^{*}(\theta, k, \mathcal{A})$ be the $(1-\theta)$ th quantile of,

$$
\int_{\tau \in \mathcal{A}} k(\tau)\left(\frac{\sqrt{N_{n}(\alpha, \varpi)}}{\left\lfloor n / k_{n}\right\rfloor m_{n}} \sum_{j=1}^{\left\lfloor n / k_{n}\right\rfloor} \sum_{i=1}^{m_{n}}\left(X_{(j-1) k_{n}+i}-\widetilde{F}_{n}(\tau)\right) v_{(j-1) k_{n}+i}^{*}\right)^{2} d \Phi(\tau) .
$$

The validity of the LWDB statistics in (24) and (25), then, follows by Lemma 2, Theorem 1 in conjunction with the same arguments provided for Theorem 3 and Corollary 2:

Theorem 4. Suppose the conditions of Theorem 3 hold. Then, locally uniformly for $\tau_{1}, \tau_{2} \in \mathcal{A}$,

$$
N_{n}(\alpha, \varpi) \mathcal{V}_{\ell, \mathrm{D}}^{*}(\mathcal{A}) \stackrel{d}{\rightarrow} \int_{\tau_{1} \in \mathcal{A}} \int_{\tau_{2} \in \mathcal{A}} \mathcal{G}_{n}\left(\tau_{1}\right) \mathcal{G}_{n}\left(\tau_{2}\right) d \ell\left(\tau_{1}, \tau_{2}\right), \quad \text { in probability- } \mathbb{P} .
$$

Corollary 3. Suppose the regularity conditions for Theorem 4 hold. Moreover, define the quantile function $Q_{n}^{*}(\theta, k, \mathcal{A})=\inf \left\{x \in \mathcal{A}: \mathbb{P}^{*}\left(\mathrm{CM}_{n}^{*}(k, \mathcal{A})>x\right) \geq \theta\right\}$. Then, for any compact subset $\mathcal{A} \subset \mathbb{R} \backslash 0$ with positive Lebesgue measure, it follows that $\mathbb{P}\left(\widehat{\mathrm{CM}}_{n}(k, \mathcal{A})>Q_{n}^{*}(\theta, k, \mathcal{A})\right) \rightarrow \theta$ as $n \rightarrow \infty$.

\section{Simulation Study}

In this section, we assess the relative finite sample properties of the Kolmogorov-Smirnov (KS) tests for local Gaussianity, $\mathcal{H}_{0}: S_{t}=W_{t}$, based on the CLT in Todorov \& Tauchen (2014) as well as our bootstrap aided-versions. Specifically, we study whether the LDWB or the NLBB can alleviate the previously reported (severe) finite sample size distortions that characterizes CLT-based test.

\subsection{Simulation Setup}

The data is simulated to match a standard 6.5-hour trading day and with the trading window normalized to the unit interval, $t \in[0,1]$, making 1 second correspond to an increment of size $1 / 23400$. In particular, we consider four different data generating processes (DGPs) in the simulations; two under $\mathcal{H}_{0}$ and two under the alternative where $S_{t}$ is a time-changed tempered stable process $\left(\mathcal{H}_{1}\right)$, allowing us to study the size and power properties of the proposed testing procedures. Specifically, for DGPs under $\mathcal{H}_{0}$, let

$$
d Z_{t}=a d t+\sigma_{t} d W_{t}+d Y_{t}, \quad d Y_{t}=\int_{\mathbb{R}} k_{0} x \mu(d t, d x),
$$

where the stochastic scale, $\sigma_{t}$, is assumed to follow a two-factor model,

$$
\sigma_{t}=\operatorname{sexp}\left(b_{0}+b_{1} \widetilde{\tau}_{1, t}+b_{2} \widetilde{\tau}_{2, t}\right) \quad \text { where } \quad d \widetilde{\tau}_{1, t}=a_{1} \widetilde{\tau}_{1, t} d t+d B_{1, t}
$$




$$
d \widetilde{\tau}_{2, t}=a_{2} \widetilde{\tau}_{2, t} d t+\left(1+\phi \widetilde{\tau}_{2, t}\right) d B_{2, t}, \quad \operatorname{Corr}\left(B_{1, t}, W_{t}\right)=\rho_{1}, \quad \operatorname{Corr}\left(B_{2, t}, W_{t}\right)=\rho_{2},
$$

and both $B_{1, t}$ and $B_{2, t}$ are standard Brownian motions, following, e.g., Chernov, Gallant, Ghysels \& Tauchen (2003) and Huang \& Tauchen (2005). ${ }^{7}$ The stochastic scale (or volatility) has two driving sources of uncertainty, two standard Brownian motions, which are correlated with $W_{t}$, thereby accommodating leverage effects. We fix the parameters in (26) and (27) as in Huang \& Tauchen (2005), that is, $\alpha=0.03, b_{0}=-1.2, b_{1}=0.04, b_{2}=1.5, a_{1}=-0.00137, a_{2}=-1.386, \phi=0.25$, as well as the correlation coefficients $\rho_{1}=\rho_{2}=-0.3$. Moreover, the two volatility factors are initialized at the onset of each "trading day" by randomly drawing the most persistent factor from its unconditional distribution, $\left.\widetilde{\tau}_{1,0} \sim N\left(0,1 /\left(2 a_{1}\right)\right)\right)$, and by letting the strongly mean-reverting factor, $\widetilde{\tau}_{2, t}$, start at zero. The two DGPs under $\mathcal{H}_{0}$, capturing the size of the tests, differ with respect to the specification of the "residual" jump process in (26). In particular, $Y_{t}$, is assumed to obey either a symmetric tempered stable process (DGP 1) or a compound Poisson process (DGP 2), which have the following decompositions of their compensators $\nu_{t}^{Y}(d x)=d t \otimes \nu^{Y}(d x)$,

$$
\nu^{Y}(d x)=c_{0} \exp \left(-\lambda_{0}|x|\right)|x|^{-\left(\beta_{0}^{\prime}+1\right)} d x \quad \text { or } \quad \nu^{Y}(d x)=c_{1} \frac{\exp \left(-x^{2} /\left(2 \sigma_{1}^{2}\right)\right)}{\sqrt{2 \pi} \sigma_{1}} d x
$$

respectively. For the symmetric tempered stable, $c_{0}>0, \lambda_{0}>0$ and $\beta_{0}^{\prime} \in[0,1)$ measures the degree of jump activity. Moreover, we follow Todorov (2009) and Hounyo \& Varneskov (2017) and let $\left(\beta_{0}^{\prime}, k_{0}, c_{0}, \lambda_{0}\right)=(0.1,0.0119,0.125,0.015)$. This model is calibrated such that the variation of $Y_{t}$ accounts for $10 \%$ of the average quadratic variation of $Z_{t}$, reflecting the empirical results in Huang \& Tauchen (2005). Similarly, $\left(c_{1}, \sigma_{1}\right)=(1,3 / 2)$ is fixed for the mean-zero, normally distributed, compound Poisson jumps (which have activity index $\beta^{\prime}=0$ ).

Under the alternative hypothesis, we let

$$
Z_{t}=S_{T_{t}}, \quad \text { with } \quad T_{t}=\int_{0}^{t} \sigma_{s}^{2} d s
$$

where $S_{t}$ is a symmetric tempered stable martingale with Levy measure $\exp (-0.25|x|)|x|^{-(1.51+1)}$ and for the stochastic time change, $T_{t}, \sigma_{t}$ is specified as in (27). The parameters of $S_{t}$ are chosen such that it behaves locally like a stable process with $\beta=1.51 .^{8}$ We either add no residual jumps to the model under the alternative (29) (DGP 3) or compound Poisson jumps as in (28) (DGP 4).

After having simulated $Z_{t_{i}}$, we construct equidistant samples $t_{i}=i / n$ for $i=0, \ldots n$ and generate returns $\Delta_{i}^{n} Z=Z_{t_{i}}-Z_{t_{i-1}}$. Specifically, we study the performance of the tests for three different samples sizes: $n=\{78,195,390\}$, corresponding to sampling every $\{5,2,1\}$ minutes, respectively. The tests require the selection of tuning parameters, $k_{n}, m_{n}, \alpha, \varpi$ and, specific to the bootstrap tests, the

\footnotetext{
${ }^{7}$ The function "sexp" is, following the literature, defined as an exponential with a linear growth function splined in at high values of its $\operatorname{argument}: \operatorname{sexp}(x)=\exp (x)$ if $x \leq x_{0}$ and $\operatorname{sexp}(x)=\frac{\exp \left(x_{0}\right)}{\sqrt{x_{0}}} \sqrt{x_{0}-x_{0}^{2}+x^{2}}$ if $x>x_{0}$ with $x_{0}=\ln (1.5)$.

${ }^{8}$ Following Todorov et al. (2014), $S_{t}$ is generated as the difference between two spectrally positive tempered stable processes, which are simulated using the acceptance-rejection algorithm of Baeumer \& Meerschaert (2009).
} 
dependence parameter $b_{n}$. By Assumption 2(b), we have $\sqrt{n} / k_{n} \rightarrow \infty$. Hence, similarly to Todorov \& Tauchen (2014), we let $\sqrt{n} / k_{n}=\varrho_{1}$, with $\varrho_{1}=\{1,5 / 4\}, m_{n} / k_{n}=0.7$ and, for the truncation of the increments, $\alpha=3$ and $\varpi=0.49$. For the implementation of the LDWB, we determine dependence of the external random variable through $b_{n} / m_{n}=\varrho_{2}$ with moderate selections $\varrho_{2}=\{1 / 2,1 / 3\}$. Using this dependence parameter, we consider four different external random variables:

DWB1: $v_{i}^{*} \sim$ i.i.d. $N(0,1)$.

DWB2: The Rademacher (i.e., the two point) distribution: $v_{i}^{*} \sim$ i.i.d. such that

$$
v_{i}^{*}= \begin{cases}1 & \text { with probability } \mathcal{P}=1 / 2, \\ -1 & \text { with probability } 1-\mathcal{P}\end{cases}
$$

DWB3: Ornstein-Uhlenbeck process $v_{i}^{*}=e^{-1 / b_{n}} v_{i-1}^{*}+\xi_{i}$, with $\xi_{i} \sim$ i.i.d. $N\left(0,1-e^{-2 / b_{n}}\right)$.

DWB4: Moving average process $v_{i}^{*}=\varsigma_{i}+\cdots+\varsigma_{i-b_{n}+1}$, where $\varsigma_{i} \sim$ i.i.d. $N\left(0,1 / b_{n}\right)$.

Note that the four choices of $v_{i}^{*}$ are asymptotically valid, satisfying Assumption DWB, and their different dependence structures allow us to assess robustness features of the LDWB. ${ }^{9}$ Moreover, we implement NLBB as a final alternative, which, as explained in Section 3.3, is nested in our LDWB procedure. All KS tests for local Gaussianity are implemented over the set,

$$
\mathcal{A}=[Q(0.001): Q(0.499)] \cup[Q(0.501): Q(0.999)],
$$

where $Q(\theta)$ is the $(1-\theta)$ th quantile of the standard normal distribution, and adopts a nominal $5 \%$ rejection level. Finally, the simulation study is carried out using 999 bootstrap samples for each of the 1000 Monte Carlo replications. The rejection rates of $\mathcal{H}_{0}: S_{t}=W_{t}$ are reported in Table 1 for the DGPs 1 and 2 (size) and Table 2 for the DGPs 3 and 4 (power). ${ }^{10}$

\subsection{Simulation Results}

There are several interesting results from Table 1. First, consistent with the evidence in Todorov \& Tauchen (2014), we find that the CLT-based KS test is (severely) oversized, especially when the local window for spot volatility estimation is $\varrho_{1}=5 / 4$. Moreover, for DGP 1 in particular, the size distortions are essentially unaffected by an increase in sample size from $n=78$ to $n=360$. Second, the LDWB-aided KS tests have much better size properties for all combinations $n, \varrho_{1}, \varrho_{2}$, DGP and external random variables. For example, if considering DGP1, $n=360$ and $\varrho_{1}=5 / 4$, the CLT rejects $21.1 \%$ of the time, whereas the LDWB1 is very close to the nominal $5 \%$ level with a rejection rate of

\footnotetext{
${ }^{9}$ The Rademacher distribution, proposed for bootstraps by Liu (1988), is advocated by Davidson \& Flachaire (2008) in the context of wild bootstrap inference for regression parameters. We assess its prowess in the case of empirical CDF inference for semimartingales at high sampling frequencies using our LDWB methodology.

${ }^{10}$ Implementation details are provided in Appendix C.
} 
6.1\%. Third, the NLBB performs slightly worse than the LDWBs, in small samples when $\varrho_{1}=1$ and more generally when $\varrho_{1}=5 / 4$, showing the benefits of our general bootstrap framework.

From the results in Table 2, we observe that all tests have power to reject the null hypothesis when false. Moreover, the rejection properties dramatically improve when the sample size is increased from $n=78$ to $n=195$, attaining full power when sampling every minute, i.e., when $n=390$. The rejection rates for the CLT-based test are slightly higher than the corresponding for bootstrap tests, especially when $n=78$ and $n=195$. However, as emphasized by Horowitz \& Savin (2000) and Davidson \& MacKinnon (2006), these results are misleading since CLT test suffers from severe size distortions for all sampling frequencies and DGPs considered. Finally, the power properties are very similar across bootstraps, albeit with the NLBB performing slightly worse than the LDWBs

In general, the simulation results demonstrate the usefulness of our bootstrap framework, restoring the size properties of tests for local Gaussianity, while maintaining excellent finite sample power.

\section{Empirical Analysis}

We consider two empirical applications to illustrate the usefulness of our new bootstrap inference and testing techniques. First, we test for local Gaussianity in high-frequency (HF) futures data on three different asset classes; equity indices, foreign exchange rates and commodities. Second, we demonstrate that the bootstrap procedures are not only applicable to HF data, but may be applied more generally as a nonparametric heteroskedasticity-robust test for local Gaussianity. To this end, we use daily data to test the distributional properties of four series that are widely used in the macro finance literature, namely the VIX, TIPS (inflation-linked bonds), default spread, and the term spread. Finally, due to the similarities between the properties of the LDWB tests in Tables 1 and 2, we focus on the differences between the CLT test and LDWB1 for simplicity of exposition. ${ }^{11}$

\subsection{High-frequency Application}

We study the null hypothesis, $\mathcal{H}_{0}: S_{t}=W_{t}$, using high-frequency data from 2010-2013 on eight futures series covering three asset classes. This presents an interesting and diverse sampling period with substantial market turbulence during the first two years, culminating in April-May 2010 with the downgrade of Greece's sovereign deb to junk bond status as well as in August 2011 where stock prices dropped sharply in fear of contagion of the European sovereign debt crises to Italy and Spain, and two calmer years during 2012-2013. Specifically, since, e.g., Andersen et al. (2015) and Hounyo \& Varneskov (2017) cannot reject $\mathcal{H}_{0}$ for S\&P 500 futures, we extend their evidence to two different equity indices, namely the DAX and FTSE 100. Moreover, we consider futures contracts on gold and oil as well as four exchange rates; the Canadian Dollar (CAD), Swiss Franc (CHF), British Pound (GBP), and the Japanese Yen (JPY), all measured against the U.S. Dollar (USD). The series are

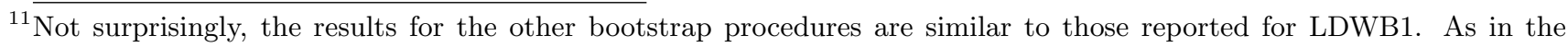
simulation study, the tests are implemented with $\varrho_{1}=1, m_{n} / k_{n}=0.7$ and 999 bootstrap samples for each series.
} 
obtained from Tick Data, include observations from both pit and electronic trading, and are sampled every minute. We use observations from 9.00 to 18.30 CET on the equity futures, 9.00 to 20.00 CET on the two commodities, and from 1.00 to $23.00 \mathrm{CET}$ on the exchange rates since the latter is traded round-the-clock, whereas the trading is sparser in the other contracts outside of regular European and U.S. market hours. ${ }^{12}$ Since these futures contracts are very liquid, minimizing concerns about market microstructure noise effects, we construct series of 1,2 and 5-minute logarithmic returns. For each series, we report the rejection rates of $\mathcal{H}_{0}$ by year using a $5 \%$ nominal level. The test results are presented in Table 3 (equity indices and commodities) and 4 (exchange rates).

From Table 3, we see that $\mathcal{H}_{0}$ is rarely rejected for the different combinations of either equity index or commodity and sampling frequency, suggesting that leading term in these assets is a Brownian motion. For the DAX and FTSE 100 indices, this evidence corroborates prior findings for the S\&P 500. Interestingly, we find very similar results for gold and oil; the tests fail to reject local Gaussianity. Second, we observe the LDWB1 test to reject uniformly less than the CLT test. For example, for FTSE futures and a 5-minute sampling frequency, the CLT test has an average reject rate of $11.2 \%$, compared to $3.2 \%$ for LDWB1. The differences in rejection rates are consistent with the simulation study; our LDWB1 test has an accurate size, whereas the CLT-based test is generally oversized, even in relatively large samples. Third, the conclusions based on 1, 2, and 5-minute are very similar, suggesting that there is no issues with market microstructure noise at these sampling frequencies. ${ }^{13}$

When turning to the results for exchange rates in Table 4, they are markedly different from those in Table 3, however, similar across currencies. The tests rarely reject for the 5-minute sampling frequency, but the rejection rates uniformly increase as the sampling frequency increase, rejecting, on average over the whole sample, $91.6 \%, 98.3 \%, 67.4 \%$, and $41.8 \%$ of the days for 1-minute observations and the CAD, CHF, GBP, and JPY, respectively. This strongly suggests that exchange rates are locally driven by a stable process with $\beta<2$, not a diffusion. Moreover, the rejection rates differ across the sample, further suggesting that there may be important time-variation in $\beta$. To corroborate these findings, we depict estimates of $\beta$ in Figure 2 for each sampling frequency using the empirical characteristic function approach of Todorov (2015). ${ }^{14}$ The $\beta$ estimates are remarkably similar across both currencies and sampling frequencies; being in the 1.75-1.90 range. Moreover, and together with the simulated power results in Table 2, they help explain the differences in rejection rates across sampling frequencies in Table 4. For sparsely sampled frequencies, our test simply lacks the power to reject $\mathcal{H}_{0}$. However, as the power properties improves with sample size, the (bootstrap) tests reject local Gaussianity almost $100 \%$ of the days for the CAD and CHF series. One may be concerned that the rejection results in Table 4 are due to microstructure noise, not the innovations being locally stable. However, the

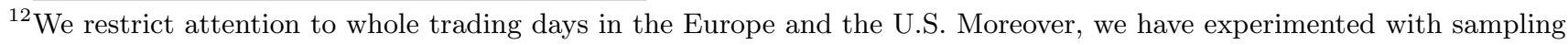
using different trading hours. The conclusions are qualitatively identical to those presented below.

${ }^{13}$ Microstructure noise represents a different rejection of $\mathcal{H}_{0}$, see Todorov \& Tauchen (2014, Theorem 2). Hence, if noise affects 1-minute observations, but not sparser sampled ones, we would expect the rejection rates to differ.

${ }^{14}$ For specificity, for each trading day, we implement the estimator in Todorov $(2015,(5.1))$ with, in his notation, the tuning parameter selections $p=0.51, u=0.25, v=0.5$, and $k_{n}=\{50,75,100\}$ for 5,2 , and 1-minute returns. From these, the median estimate for a given calendar month is computed and depicted for all three sampling frequencies.
} 
combination of results in Tables 2 and 4 as well as the similarity of the $\beta$ estimates across sampling frequencies in Figure 2 deem the (lack of) power hypothesis for the tests more likely. Of course, to rigorously dismiss the market microstructure noise hypothesis, it would require a noise-robust version of the LDWB1 test. We leave this for further research. ${ }^{15}$

In general, we find that equity indices and commodities (gold and oil) are well-described as locally Gaussian, whereas exchange rates are better approximated by locally stable innovations with stability index $\beta$ in the 1.80-1.90 range. As explained in the introduction, these differences across asset classes hold important implications for model specification, risk measures and derivatives pricing.

\subsection{Macro Finance Application}

The empirical macro finance literature often use various financial variables to predict future economic conditions, cross-sectional asset pricing and to describe the dynamics of consumption growth, among others. To show that our bootstrap inference and testing procedures apply generally, we test whether four key variables are described by (locally) Gaussian innovations, possibly in conjunction with a stochastic scale (volatility), using daily data from 2006 through 2017, amounting to 3130 observations. Whereas the VIX and TIPS series are obtained straightforwardly, the default spread is constructed as the difference between logarithmic yields on Moody's BAA and AAA bonds, and the term spread is constructed as the difference between the log prices of generic first futures contracts on 10-year and 2-year US Treasury notes. We obtain log-returns for the VIX and TIPS series, and first differences for the default and term spreads. The series are displayed in Figure 3.

Similar to returns on various assets, for example, as analyzed above, equity indices, commodities, and exchange rates, the series display stochastic and clustering volatility. Hence, the nonparametric standardization in the (bootstrap) testing procedure is important for determining whether the innovations are locally Gaussian. As a second step, we depict the empirical CDFs of the respective standardized and truncated innovations in Figure 4 against a standard Gaussian CDF. The visual evidence suggests that CDFs for the VIX and term spread deviate from a standard Gaussian one, however, that the corresponding for the TIPS and default spread are very close. Indeed, when testing $\mathcal{H}_{0}$ using the LDWB1-aided test, we reject for the VIX - consistent with Todorov \& Tauchen (2011b), Andersen et al. (2015) and Hounyo \& Varneskov (2017) - and for the term spread series, but not for the remaining two. However, when applying the CLT test, we reject $\mathcal{H}_{0}$ for all four series. If we compare the critical value for the CLT, 1.28 , to the test statistics $\{3.29,1.46,1.38,2.21\}$, we observe that the rejections for the TIPS and default spread (2 and 3) are borderline, whereas local Gaussianity of the VIX and term spread (1 and 4) are strongly rejected. In contrast, the corresponding LDWB1

\footnotetext{
${ }^{15}$ The evidence in Table 4 is consistent with the findings in Todorov \& Tauchen (2010) and Cont \& Mancini (2011), who, using 5-minute observations on the DM-USD exchange rate from the 1990s, argue that exchange rates are locally Gaussian. These test may simply lack the power to reject $\mathcal{H}_{0}$, as for the 5 -minute series in Table 4 . Moreover, Hounyo \& Varneskov (2017) find rejection rates of $\beta=2$ for currencies to be between $20-56 \%$ using a bootstrap-aided realized power variation test. Our results, using the LDWB1, are much stronger, which speaks directly to power differences between tests based on either the empirical CDF and power variation measures, see Todorov \& Tauchen (2014, Table 2).
} 
critical values are $\{1.68,1.54,1.85,1.52\}$, overturning the conclusions for the TIPS and default spread. The different results are attributed to the size problems associated with the CLT test, even in large samples. Our bootstrap test, on the other hand, has excellent size and maintain $\mathcal{H}_{0}$ in the two cases, which, by Figure 4, is consistent with visual evidence for the respective empirical CDFs.

\section{Conclusion}

This paper provides a new inference procedure for the local innovation of Itô semimartingales. Specifically, we construct a resampling procedure for the empirical CDF of high-frequency innovations that have been standardized using a nonparametric estimate of its stochastic scale (volatility) and truncated to rid the effect of "large" and more infrequent jumps. Our locally dependent wild bootstrap (LDWB) accommodate issues related to the stochastic scale and jumps as well as account for a special block-wise dependence structure induced by sampling errors arising from having replaced the stochastic scale with a nonparametric estimate. We show that the LDWB replicates first and second-order limit theory from the usual empirical process component of the statistic and the stochastic scale estimate, respectively, in addition to an asymptotic bias. Moreover, we design the LDWB sufficiently general to establish asymptotic equivalence between it and and a nonparametric local block bootstrap, also introduced here, up to second-order distribution theory, providing new theoretical insights into the relation between bootstrap paradigms. Finally, we introduce LDWB-aided Kolmogorov-Smirnov tests for local Gaussianity as well as local von-Mises statistics, with and without accompanying bootstrap inference, and establish their asymptotic validity using the second-order distribution theory.

The finite sample performance of CLT and LDWB-aided local Gaussianity tests are assessed in a simulation study as well as two empirical applications to high-frequency futures data and popular macro finance variables. Whereas the CLT test is oversized, even in large samples, the size of the LDWB tests are accurate, even in small samples. Moreover, the gains in size are without loss of power, even in moderate sample sizes. The empirical analysis verifies this pattern, the CLT tests rejects uniformly more often the the LDWB test for assets that are well-described as locally Gaussian such as equity indices and commodities. Moreover, it shows that local Gaussianity is strongly rejected for exchange rate series, which, in contrast, are better described as locally stable with tail index in the 1.80 to 1.90 range. Finally, when applying the test to macro finance variables such as the VIX, TIPS, default spread and term spread, we show that the CLT test erroneously rejects for all four series, whereas the LDWB rejects for the VIX and term spread series, in line with visual evidence. 


\begin{tabular}{|c|c|c|c|c|c|c|c|c|}
\hline \multicolumn{9}{|c|}{ Rejection Rates under $\mathcal{H}_{0}$} \\
\hline & \multicolumn{8}{|c|}{$\varrho_{1}=1$} \\
\hline & CLT & NLBB & LDWB1 & LDWB2 & $\mathrm{LDWB}_{1 / 2}$ & $\mathrm{LDWB}_{1 / 3}$ & $\mathrm{LDWB}_{1 / 2}$ & $\mathrm{LDWB}_{1 / 3}$ \\
\hline \multicolumn{9}{|l|}{ DGP 1} \\
\hline$n=78$ & 17.4 & 9.6 & 4.0 & 4.5 & 7.2 & 6.0 & 5.0 & 3.7 \\
\hline$n=195$ & 13.6 & 8.1 & 3.9 & 4.5 & 6.1 & 5.5 & 4.5 & 4.4 \\
\hline$n=390$ & 13.1 & 6.0 & 3.2 & 3.3 & 5.4 & 4.8 & 4.5 & 3.8 \\
\hline \multicolumn{9}{|l|}{ DGP 2} \\
\hline$n=78$ & 16.1 & 9.2 & 3.2 & 4.4 & 6.9 & 4.7 & 4.1 & 3.2 \\
\hline$n=195$ & 12.0 & 5.3 & 2.4 & 3.0 & 4.5 & 4.0 & 2.9 & 2.9 \\
\hline \multirow[t]{3}{*}{$n=390$} & 7.8 & 4.0 & 2.0 & 2.2 & 3.3 & 3.1 & 2.3 & 2.2 \\
\hline & \multicolumn{8}{|c|}{$\varrho_{1}=5 / 4$} \\
\hline & CLT & NLBB & LDWB1 & LDWB2 & $\mathrm{LDWB}_{1 / 2}$ & $\mathrm{LDWB}_{1 / 3}$ & $\mathrm{LDWB}_{1 / 2}$ & $\mathrm{LDWB}_{1 / 3}$ \\
\hline \multicolumn{9}{|l|}{ DGP 1} \\
\hline$n=78$ & 24.6 & 13.6 & 6.9 & 9.0 & 12.8 & 9.5 & 8.9 & 7.4 \\
\hline$n=195$ & 19.7 & 9.1 & 3.8 & 4.9 & 6.9 & 5.7 & 5.2 & 4.7 \\
\hline$n=390$ & 21.1 & 10.5 & 6.1 & 6.0 & 9.8 & 8.1 & 7.7 & 6.8 \\
\hline \multicolumn{9}{|l|}{ DGP 2} \\
\hline$n=78$ & 24.2 & 12.1 & 5.8 & 7.6 & 11.4 & 8.2 & 7.5 & 6.3 \\
\hline$n=195$ & 18.4 & 9.3 & 5.8 & 6.9 & 7.9 & 6.4 & 6.2 & 5.8 \\
\hline$n=390$ & 19.0 & 8.3 & 5.3 & 5.5 & 7.5 & 6.6 & 6.1 & 5.4 \\
\hline
\end{tabular}

Table 1: Size results. This table provides rejection frequencies of the null hypothesis $\mathcal{H}_{0}: S_{t}=W_{t}$ for DGPs 1 and 2, sample sizes $n=\{78,195,390\}$, as well as eight different tests; CLT, NLBB, LDWB1, LDWB2, LDWB3, and LDWB4. In particular, CLT denotes the Kolmogorov-Smirnov (KS) test in (11), see also Todorov \& Tauchen (2014), NLBB is the nonparametric local block bootstrap described in Section 3.3, and LDWB with numbers 1-4 are different implementations of the locally dependent wild bootstrap in Section 3.2, see Theorems 1 and 2 as well as Section 5. The numbers refer to different external random variables: (1) Gaussian; (2) Rademacher; (3) Ornstein-Uhlenbeck and (4) Moving average. For LDWB3 and LDWB4, the subscript refers to $\varrho_{2}=\{1 / 2,1 / 3\}$, capturing their dependence structures. The nominal level of the KS tests is $5 \%$. Finally, the exercise is performed for 999 bootstrap samples for every one of the 1000 Monte Carlo replications. 


\begin{tabular}{|c|c|c|c|c|c|c|c|c|}
\hline \multicolumn{9}{|c|}{ Rejection Rates under $\mathcal{H}_{1}$} \\
\hline & \multicolumn{8}{|c|}{$\varrho_{1}=1$} \\
\hline & CLT & NLBB & LDWB1 & LDWB2 & $\mathrm{LDWB}_{1 / 2}$ & $\mathrm{LDWB}_{1 / 3}$ & $\mathrm{LDWB}_{1 / 2}$ & $\mathrm{LDWB}_{1 / 3}$ \\
\hline \multicolumn{9}{|l|}{ DGP 3} \\
\hline$n=78$ & 59.3 & 36.5 & 35.8 & 38.6 & 41.3 & 39.0 & 36.5 & 36.2 \\
\hline$n=195$ & 95.0 & 83.1 & 84.7 & 85.6 & 86.2 & 85.8 & 84.4 & 84.3 \\
\hline$n=390$ & 99.7 & 98.7 & 99.3 & 99.3 & 99.0 & 99.1 & 99.0 & 99.1 \\
\hline \multicolumn{9}{|l|}{ DGP 4} \\
\hline$n=78$ & 56.4 & 36.3 & 36.5 & 39.0 & 41.3 & 38.4 & 36.2 & 36.0 \\
\hline$n=195$ & 94.3 & 79.9 & 83.7 & 84.3 & 83.6 & 83.8 & 82.8 & 82.4 \\
\hline \multirow[t]{3}{*}{$n=390$} & 99.9 & 99.0 & 99.7 & 99.7 & 99.5 & 99.6 & 99.7 & 99.7 \\
\hline & \multicolumn{8}{|c|}{$\varrho_{1}=5 / 4$} \\
\hline & CLT & NLBB & LDWB1 & LDWB2 & $\mathrm{LDWB}_{1 / 2}$ & $\mathrm{LDWB}_{1 / 3}$ & $\mathrm{LDWB}_{1 / 2}$ & $\mathrm{LDWB}_{1 / 3}$ \\
\hline \multicolumn{9}{|l|}{ DGP 3} \\
\hline$n=78$ & 60.2 & 39.1 & 39.2 & 42.3 & 44.2 & 42.5 & 39.7 & 39.7 \\
\hline$n=195$ & 92.2 & 77.1 & 81.2 & 81.3 & 81.3 & 81.4 & 79.4 & 80.4 \\
\hline$n=390$ & 99.8 & 99.2 & 99.0 & 98.9 & 98.8 & 98.8 & 98.8 & 98.9 \\
\hline \multicolumn{9}{|l|}{ DGP 4} \\
\hline$n=78$ & 62.2 & 40.1 & 42.0 & 44.8 & 47.6 & 44.5 & 41.3 & 42.3 \\
\hline$n=195$ & 91.0 & 76.5 & 80.0 & 80.6 & 79.8 & 79.6 & 79.0 & 79.5 \\
\hline$n=390$ & 99.7 & 98.7 & 99.0 & 98.8 & 98.8 & 98.9 & 98.8 & 98.8 \\
\hline
\end{tabular}

Table 2: Power results. This table provides rejection frequencies of the null hypothesis $\mathcal{H}_{0}$ for DGPs 3 and 4, sample sizes $n=\{78,195,390\}$, as well as eight different tests; CLT, NLBB, LDWB1, LDWB2, LDWB3, and LDWB4. In particular, CLT denotes the Kolmogorov-Smirnov (KS) test in (11), see also Todorov \& Tauchen (2014), NLBB is the nonparametric local block bootstrap described in Section 3.3, and LDWB with numbers 1-4 are different implementations of the locally dependent wild bootstrap in Section 3.2, see Theorems 1 and 2 as well as Section 5. The numbers refer to different external random variables: (1) Gaussian; (2) Rademacher; (3) Ornstein-Uhlenbeck and (4) Moving average. For LDWB3 and LDWB4, the subscript refers to $\varrho_{2}=\{1 / 2,1 / 3\}$, capturing their dependence structures. The nominal level of the KS tests is $5 \%$. Finally, the exercise is performed for 999 bootstrap samples for every one of the 1000 Monte Carlo replications. 


\begin{tabular}{|c|c|c|c|c|c|c|c|c|}
\hline \multicolumn{9}{|c|}{ Rejection Rates for Equity Indices and Commodities } \\
\hline & \multicolumn{2}{|c|}{2010} & \multicolumn{2}{|c|}{2011} & \multicolumn{2}{|c|}{2012} & \multicolumn{2}{|c|}{2013} \\
\hline & CLT & LDWB1 & CLT & LDWB1 & CLT & LDWB1 & CLT & LDWB1 \\
\hline \multicolumn{9}{|l|}{ DAX } \\
\hline 1 -min & 4.31 & 1.18 & 7.03 & 1.95 & 4.74 & 0.40 & 3.56 & 0.00 \\
\hline 2 -min & 5.49 & 0.39 & 7.81 & 1.56 & 5.14 & 0.40 & 2.77 & 0.00 \\
\hline 5 -min & 7.84 & 0.00 & 10.94 & 1.95 & 6.32 & 1.98 & 7.11 & 0.79 \\
\hline \multicolumn{9}{|l|}{ FTSE } \\
\hline $1-\min$ & 8.37 & 2.79 & 8.43 & 2.81 & 5.20 & 1.60 & 7.97 & 1.99 \\
\hline 2 -min & 9.16 & 1.20 & 5.62 & 2.01 & 8.00 & 1.60 & 2.79 & 0.40 \\
\hline 5 -min & 9.16 & 3.19 & 16.06 & 4.82 & 7.60 & 1.20 & 11.95 & 3.59 \\
\hline \multicolumn{9}{|l|}{ Gold } \\
\hline 1-min & 6.59 & 1.16 & 5.81 & 2.33 & 4.65 & 0.78 & 5.43 & 1.16 \\
\hline 2-min & 3.10 & 0.39 & 2.71 & 0.39 & 2.71 & 0.78 & 6.98 & 0.39 \\
\hline 5 -min & 6.98 & 1.16 & 6.98 & 1.55 & 3.49 & 0.00 & 4.26 & 0.78 \\
\hline \multicolumn{9}{|l|}{ Oil } \\
\hline 1-min & 5.14 & 1.19 & 7.78 & 3.50 & 3.88 & 1.16 & 4.65 & 1.55 \\
\hline 2 -min & 3.95 & 1.19 & 3.11 & 0.00 & 6.98 & 0.39 & 3.49 & 0.00 \\
\hline 5 -min & 11.07 & 1.98 & 7.78 & 0.39 & 8.91 & 1.16 & 8.53 & 1.16 \\
\hline
\end{tabular}

Table 3: Empirical rejection rates. This table provides rejection frequencies of the null hypothesis $\mathcal{H}_{0}: S_{t}=W_{t}$ for the CLT and LDWB1 tests. In particular, CLT denotes the Kolmogorov-Smirnov (KS) test in (11), see also Todorov \& Tauchen (2014), and LDWB1 is the locally dependent wild bootstrap-based test with standard Gaussian external random variables, see Theorems 1 and 2 as well as Section 5 . The tests are implemented on high-frequency futures data from both pit and electronic trading for the DAX and FTSE 100 equity indices as well as Gold and Oil futures. Three different sampling frequencies are considered; every 1, 2, and 5 minutes. For the equity index futures, the trading hours are 9.00-18.30 (CET), amounting to sample sizes $n=\{570,285,114\}$ for the three sampling frequencies. For the commodity futures, the trading hours are 9.00-20.00 (CET), amounting to sample sizes $n=\{660,330,132\}$ for the three sampling frequencies. The nominal level of the KS tests is $5 \%$. Finally, we use 999 replications for the bootstrap resampling, as in the simulation study. 


\begin{tabular}{|c|c|c|c|c|c|c|c|c|}
\hline \multicolumn{9}{|c|}{ Rejection Rates for Currencies } \\
\hline & \multicolumn{2}{|c|}{2010} & \multicolumn{2}{|c|}{2011} & \multicolumn{2}{|c|}{2012} & \multicolumn{2}{|c|}{2013} \\
\hline & CLT & LDWB1 & CLT & LDWB1 & CLT & LDWB1 & CLT & LDWB1 \\
\hline \multicolumn{9}{|c|}{ CAD-USD } \\
\hline 1-min & 92.31 & 85.83 & 91.09 & 82.59 & 100 & 98.77 & 99.59 & 99.59 \\
\hline 2 -min & 32.39 & 16.19 & 38.46 & 21.05 & 69.67 & 50.00 & 91.80 & 84.02 \\
\hline 5 -min & 7.29 & 1.62 & 5.25 & 1.21 & 6.97 & 2.46 & 32.38 & 13.52 \\
\hline \multicolumn{9}{|c|}{ CHF-USD } \\
\hline 1-min & 98.79 & 96.36 & 99.19 & 97.57 & 100 & 100 & 99.59 & 99.18 \\
\hline 2 -min & 61.54 & 38.87 & 54.66 & 31.17 & 88.93 & 78.28 & 86.48 & 74.59 \\
\hline 5 -min & 6.48 & 1.21 & 2.43 & 1.21 & 7.79 & 2.46 & 10.66 & 4.51 \\
\hline \multicolumn{9}{|c|}{ GBP-USD } \\
\hline 1-min & 66.40 & 44.94 & 69.23 & 52.23 & 95.08 & 86.48 & 95.49 & 86.48 \\
\hline 2-min & 8.50 & 4.05 & 8.50 & 3.64 & 29.10 & 15.57 & 32.79 & 15.57 \\
\hline 5-min & 2.02 & 0.81 & 2.43 & 0.40 & 3.69 & 0.00 & 3.28 & 0.41 \\
\hline \multicolumn{9}{|c|}{ JPY-USD } \\
\hline 1-min & 40.49 & 21.46 & 56.68 & 40.08 & 84.02 & 73.36 & 42.31 & 32.38 \\
\hline 2-min & 6.07 & 2.02 & 14.17 & 4.45 & 22.95 & 9.43 & 9.43 & 4.51 \\
\hline 5-min & 3.24 & 0.40 & 4.45 & 0.81 & 3.28 & 0.41 & 6.56 & 1.23 \\
\hline
\end{tabular}

Table 4: Empirical rejection rates for currencies. This table provides rejection frequencies of the null hypothesis $\mathcal{H}_{0}: S_{t}=W_{t}$ for the CLT and LDWB1 tests. In particular, CLT denotes the Kolmogorov-Smirnov (KS) test in (11), see also Todorov \& Tauchen (2014), and LDWB1 is the locally dependent wild bootstrap-based test with standard Gaussian external random variables, see Theorems 1 and 2 as well as Section 5 . The tests are implemented on high-frequency futures data from both pit and electronic trading for four exchange rate futures. Three different sampling frequencies are considered; every 1, 2, and 5 minutes. For the currencies, the trading hours are 1.00-23.00 (CET), amounting to sample sizes $n=\{1320,660,264\}$ for the three sampling frequencies. The nominal level of the KS tests is 5\%. Finally, we use 999 replications for the bootstrap resampling, as in the simulation study. 
Impact of Tails

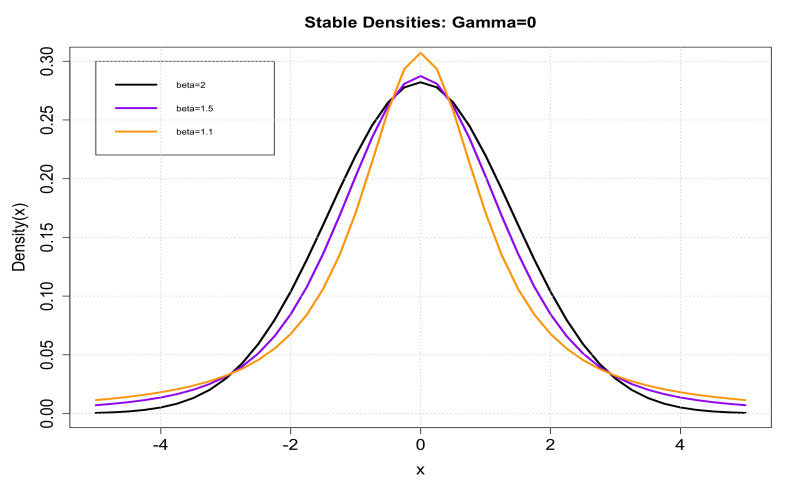

Impact of Skewness

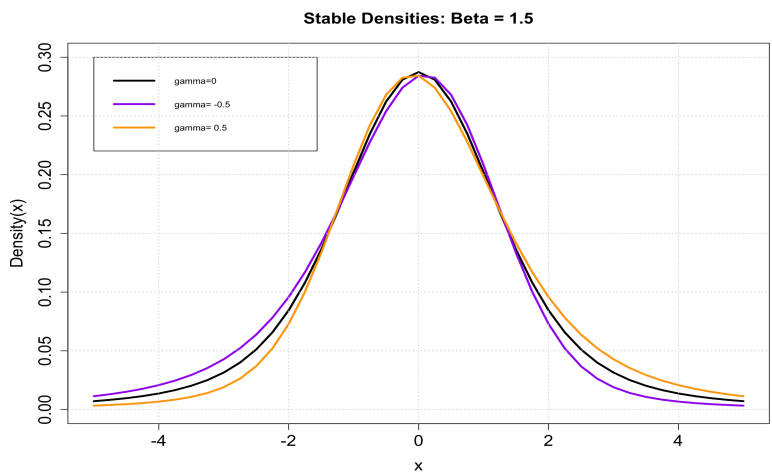

Figure 1: Stable densities. This picture illustrate the density of stable process with different values of their stability and skewness parameters, $\beta$ and $\gamma$, noting that $\beta=2$ implies a Gaussian variable. 
Activity: CAD/USD

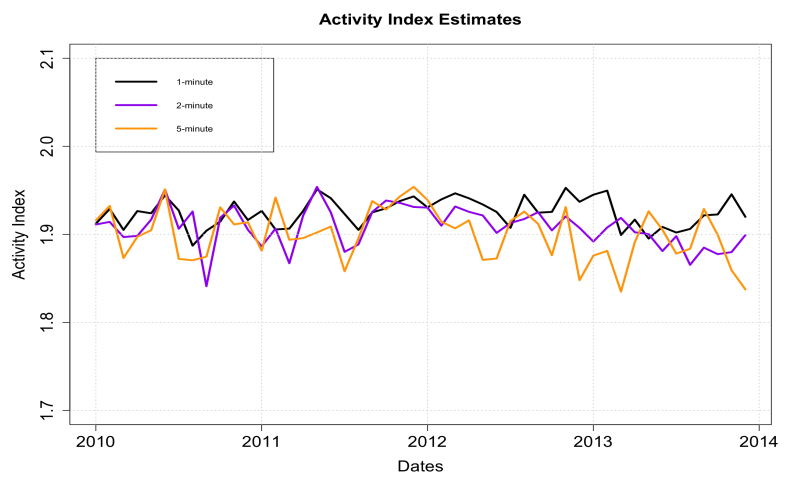

Activity: GBP/USD

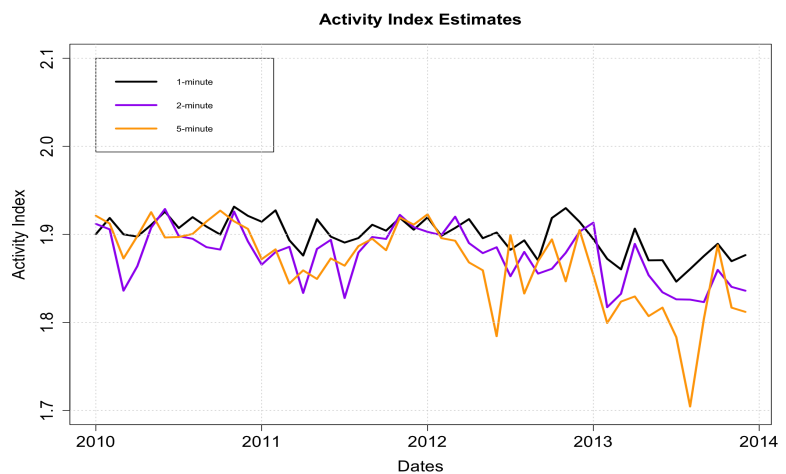

Activity: CHF/USD

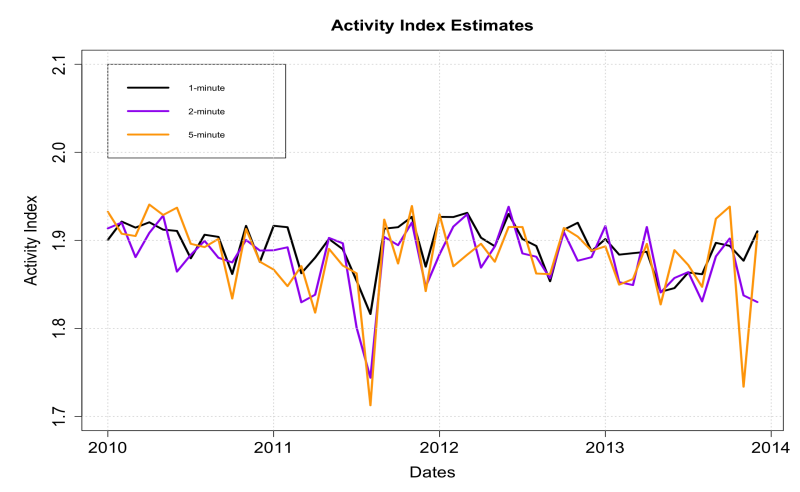

Activity: JPY/USD

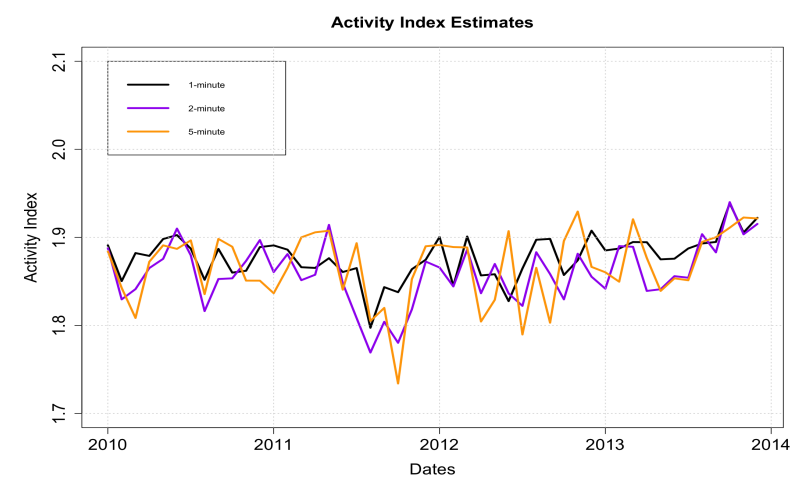

Figure 2: Activity index estimates. This picture depict daily activity index estimates for the four different exchange rates using the empirical characteristic function approach by Todorov (2015). The estimates are provided for three different sampling frequencies; 1-minute (black), 2-minute (purple), and 5-minute (orange). The estimator in Todorov $(2015,(5.1))$ is implemented with, in his notation, the tuning parameter selections $p=0.51, u=0.25$, $v=0.5$, and $k_{n}=\{50,75,100\}$ for 5,2 , and 1-minute returns. From these, the median estimate for a given calendar month in the period 2010-2013 is computed and depicted for all three sampling frequencies. 
Returns: VIX

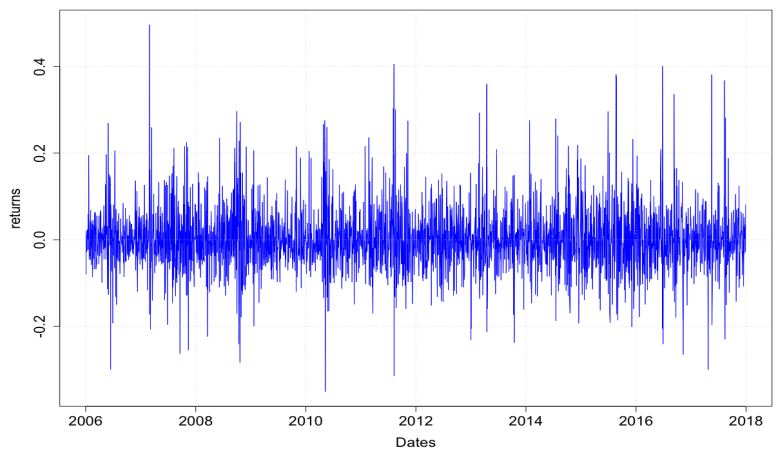

Returns: DS

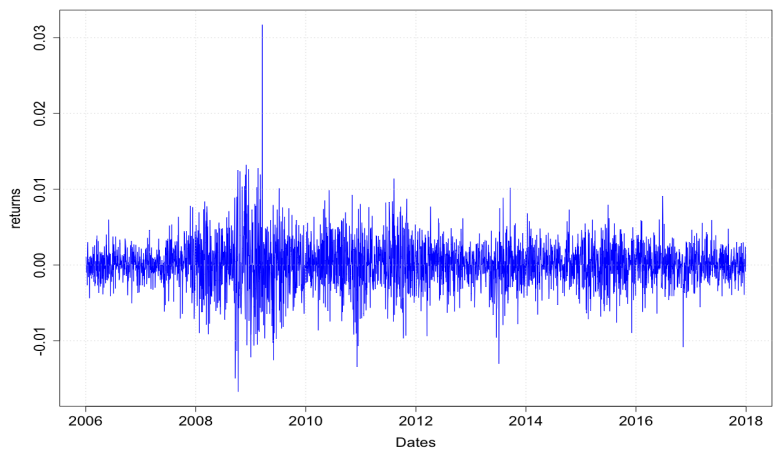

Returns: TIPS

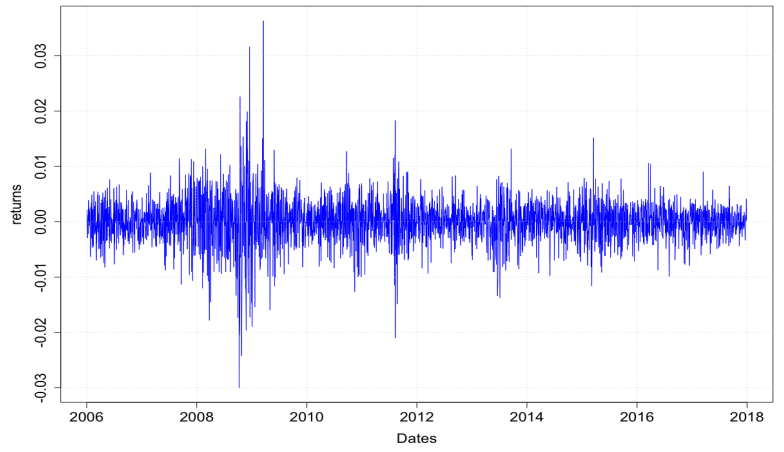

Returns: TS

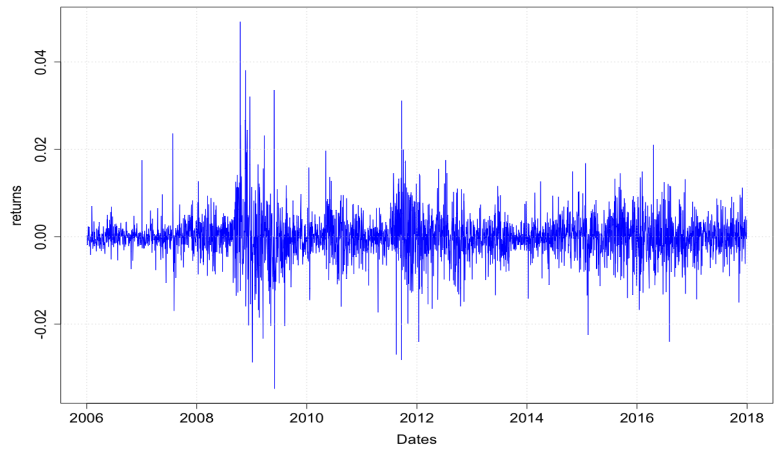

Figure 3: Return series. This picture shows the (log-)returns on the VIX, TIPS, default spread (DS) and term spread (TS) for the daily sample spanning from 2006 through 2017, amounting to $n=3130$ observations. 
VIX

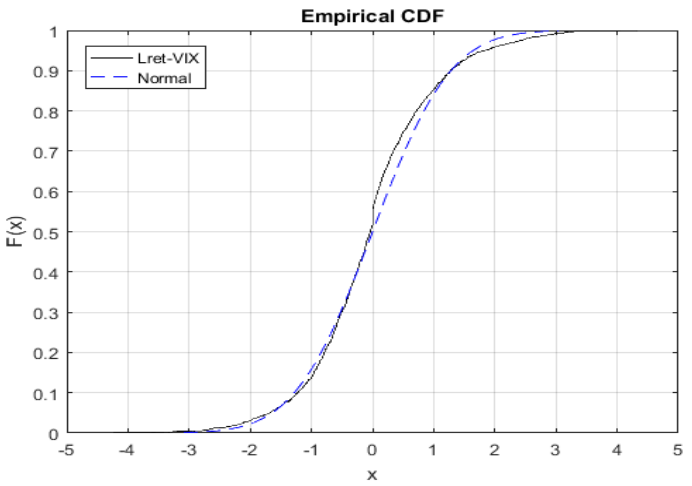

Default Spread

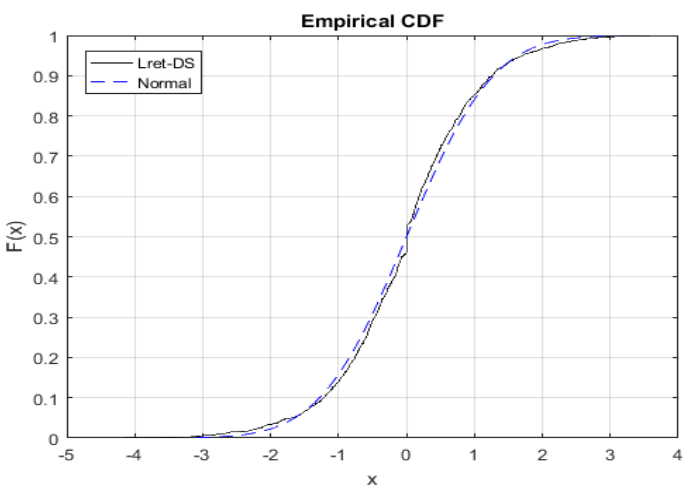

TIPS

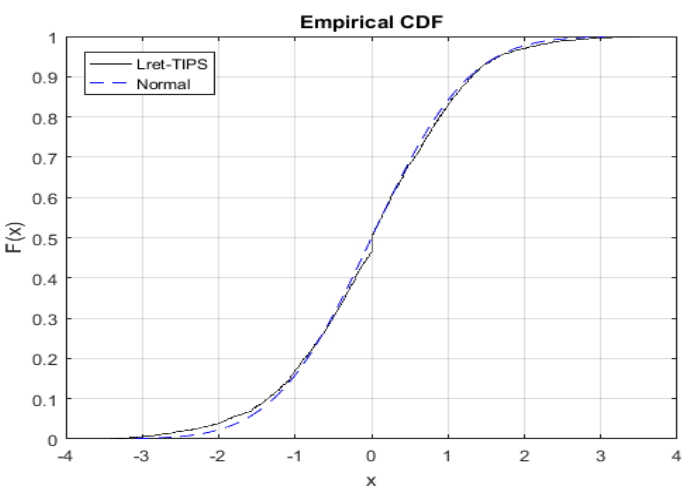

Term Spread

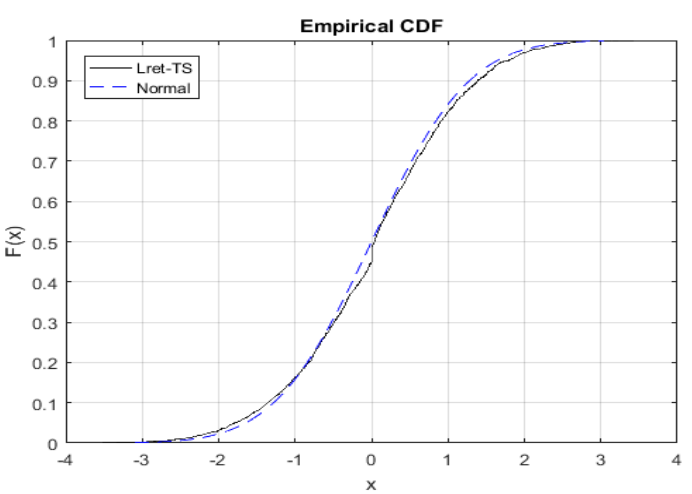

Figure 4: Empirical CDF. This picture shows the empirical CDF of the nonparametrically standardized and truncated (log-)returns on the VIX, TIPS, default spread (DS) and term spread (TS) for the daily sample spanning from 2006 through 2017, amounting to $n=3130$ observations. Note that when testing for local Gaussianity using the KS tests (CLT and LDWB1), the mass at $x=0$ is excluded from the set $\mathcal{A}$. 


\section{A Technical Results and Proofs}

This section contains additional assumptions and definitions as well as the proofs of the main asymptotic results in the paper. Before proceeding, however, let us introduce some notation. Denote by $K$ a generic constant, which may take different values from line to line or from (in)equality to (in)equality. Moreover, we write $x \wedge y=\min (x, y)$ and $x \vee y=\max (x, y)$ and adopt the following shorthand convention for subscript time indices; $\frac{(j-1) k_{n}}{n}$ signifies $t_{\underline{(j-1) k_{n}}}$. Let $\circ$ indicate the hadamard product. Finally, let us write $\mathbb{E}_{i-1}^{n}[\cdot]=\mathbb{E}\left[\cdot \mid \mathcal{F}_{(i-1) / n}\right]$ and $\mathbb{E}_{i-1}^{*}[\cdot] \stackrel{n}{=} \mathbb{E}_{i-1}^{n}\left(\mathbb{E}^{*}\left[\cdot \mid \mathcal{X}_{n}\right]\right)$ denote conditional expectations under the physical and bootstrap probability measures, respectively.

\section{A.1 Additional Assumptions}

As in Todorov \& Tauchen (2014), we shall establish the main Theorem 1 under the following stronger version of Assumption 1, and then rely on a standard localization argument, cf. Jacod \& Protter (2012, Lemma 4.4.9), to extend the results to the weaker Assumption 1.

Assumption S1. In addition to Assumption 1, the following conditions hold:

(a) $\alpha_{t}, \tilde{\alpha}_{t}, \sigma_{t}, \sigma_{t}^{-1}, \tilde{\sigma}_{t}, \tilde{\sigma}_{t}^{\prime}$ and the coefficients of the Itô semimartingale representations of $\tilde{\sigma}_{t}$ and $\tilde{\sigma}_{t}^{\prime}$ are all uniformly bounded on $t \in[0,1]$.

(b) For some negative valued function, $\phi(x)$ on the auxiliary space $E$ satisfying the regularity conditions $\int_{E} \nu(x: \phi(x) \neq 0)<\infty$ and $\phi(x) \leq K$,

$$
\left|\delta^{Y}(t, x)\right|+\left|\delta^{\sigma}(t, x)\right|+\left|\delta^{\tilde{\sigma}}(t, x)\right|+\left|\delta^{\tilde{\sigma}^{\prime}}(t, x)\right| \leq \phi(x) .
$$

\section{A.2 Additional Definitions}

We need to introduce several different quantities for the proof of the main Theorem 1. Hence, to improve exposition and ease readability of the latter, we have collected them all in this subsection as well as used the same notation as Todorov \& Tauchen (2014) when it is applicable:

- $A_{t}=\int_{0}^{t} \alpha_{s} d s$ and $B_{t}=\int_{0}^{t} \sigma_{s} d W_{s}$. Moreover, for for $j=1, \ldots,\left\lfloor n / k_{n}\right\rfloor$, let

$$
\tilde{V}_{n, j}=\frac{n}{k_{n}-1} \sum_{(j-1) k_{n}+2}^{j k_{n}}\left|\Delta_{i-1}^{n} B \| \Delta_{i}^{n} B\right|, \quad \bar{V}_{n, j}=\sigma_{\frac{(j-1) k_{n}}{n}}^{2} \frac{\pi n}{2\left(k_{n}-1\right)} \sum_{i=(j-1) k_{n}+2}^{j k_{n}}\left|\Delta_{i-1}^{n} W\right|\left|\Delta_{i}^{n} W\right| .
$$

and define $\tilde{V}_{n, j}(i)$ and $\bar{V}_{n, j}(i)$ analogously, using the same structure as in (6).

- $\tilde{V}_{n, j}-\bar{V}_{n, j}=\sum_{g=1}^{4} R_{j}^{(g)}$ where $\sum_{g=1}^{3} R_{j}^{(g)}$ will not appear explicitly in our derivations below, and we refer to Todorov \& Tauchen $(2014,(10.4))$ for definitions.

- $R_{j}^{(4)}=\frac{2}{k_{n}-1} \sigma_{\frac{(j-1) k_{n}}{n}} \sum_{(j-1) k_{n}+2}^{j k_{n}}\left[\int_{\frac{(j-1) k_{n}}{n}}^{\frac{i-2}{n}} \tilde{\sigma}_{\frac{(j-1) k_{n}}{n}} d W_{u}+\int_{\frac{(j-1) k_{n}}{n}}^{\frac{i-2}{n}} \tilde{\sigma}_{\frac{(j-1) k_{n}}{n}}^{\prime} d W_{u}^{\prime}\right]$. 
- $\tilde{R}_{i, j}^{(4)}=R_{j}^{(4)}-\frac{2}{k_{n}-1} \sigma_{\frac{(j-1) k_{n}}{n}}\left(j k_{n}-i-1\right)\left[\int_{\frac{i-1}{n}}^{\frac{i}{n}} \tilde{\sigma}_{\frac{(j-1) k_{n}}{n}} d W_{u}+\int_{\frac{i-1}{n}}^{\frac{i}{n}} \tilde{\sigma}_{\frac{(j-1) k_{n}}{n}}^{\prime} d W_{u}^{\prime}\right]$ is the component of $R_{j}^{(4)}$ that does not contain $\Delta_{i}^{n} W$ and $\Delta_{i}^{n} W^{\prime}$ for $i=(j-1) k_{n}+1, \ldots, j k_{n}-2$.

- $R_{i, j}^{(4)}(i)$ and $\tilde{R}_{i, j}^{(4)}(i)$ are the analogous components from $\tilde{V}_{n, j}(i)-\bar{V}_{n, j}(i)=\sum_{g=1}^{4} R_{j}^{(g)}(i)$.

Furthermore, for $i=(j-1) k_{n}+1, \ldots,(j-1) k_{n}+m_{n}$ and $j=1, \ldots,\left\lfloor n / k_{n}\right\rfloor$, define

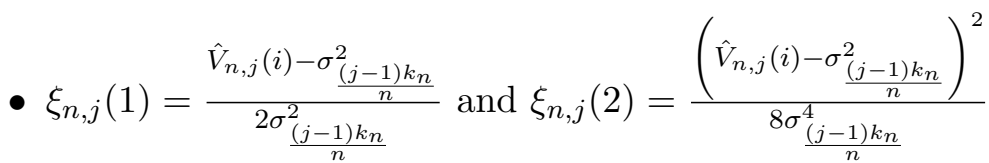

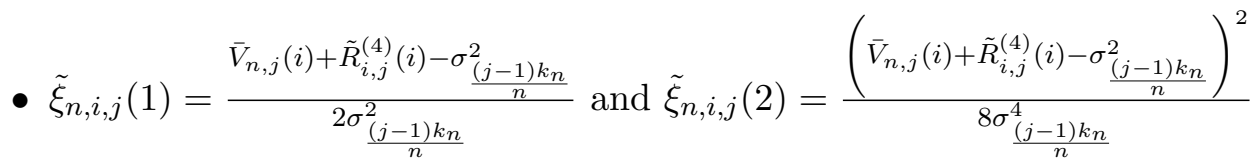

- $\bar{\xi}_{n, i, j}(1)=\frac{\bar{V}_{n, j}(i)+R_{i, j}^{(4)}-\sigma_{(j-1) k_{n}}^{2}}{n}$ and $\bar{\xi}_{n, i, j}(2)=\frac{\left(\bar{V}_{n, j}(i)+R_{i, j}^{(4)}-\sigma_{\frac{(j-1) k_{n}}{2}}^{2}\right)^{2}}{\frac{\left(\sigma^{(j-1) k_{n}}\right.}{n}}$

- $\hat{\xi}_{n, j}(1)=\frac{\bar{V}_{n, j}(i)-\sigma_{\frac{(j-1) k_{n}}{n}}^{2}}{2 \sigma^{2}{ }_{(j-1) k_{n}}^{n}}$ and $\hat{\xi}_{n, j}(2)=\frac{\left(\bar{V}_{n, j}(i)-\sigma_{\frac{(j-1) k_{n}}{n}}^{2}\right)^{2}}{8 \sigma_{(j-1) k_{n}}^{4}}$

- $\xi_{n, i, j}(3)=\frac{\sqrt{n} \Delta_{i}^{n} W}{\frac{\sigma_{(j-1) k_{n}}}{n}}\left[\tilde{\sigma}_{\frac{(j-1) k_{n}}{n}}\left(W_{\frac{i-1}{n}}-W_{\frac{(j-1) k_{n}}{n}}\right)+\tilde{\sigma}_{\frac{(j-1) k_{n}}{n}}^{\prime}\left(W_{\frac{i-1}{n}}^{\prime}-W_{\frac{(j-1) k_{n}}{n}}^{\prime}\right)\right]$.

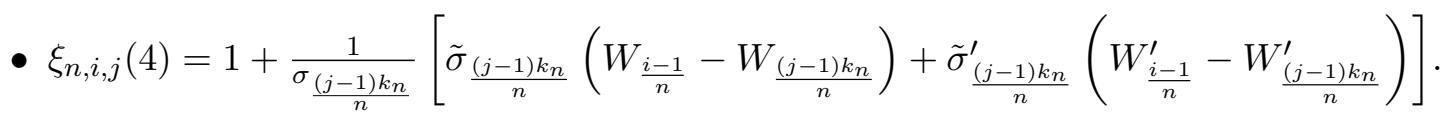

- $\chi_{n, i, j}(1)=-\chi_{n, i, j}(1,1)+\chi_{n, i, j}(1,2)-\chi_{n, i, j}(1,3)$, where

$$
\begin{aligned}
& \chi_{n, i, j}(1,1)=\sqrt{n} \frac{1}{\sigma_{\frac{(j-1) k_{n}}{n}}}\left(\Delta_{i}^{n} A+\Delta_{i}^{n} Y+\int_{\frac{i-1}{n}}^{\frac{i}{n}}\left(\sigma_{u}-\sigma_{\frac{i-1}{n}}\right) d W_{u}\right) 1\left\{\frac{\sqrt{n}\left|\Delta_{i}^{n} Z\right|}{\sqrt{\hat{V}_{n, j}}} \leq \alpha n^{1 / 2-\varpi}\right\}, \\
& \chi_{n, i, j}(1,2)=\left(\sqrt{n} \Delta_{i}^{n} W+\xi_{n, i, j}(3)\right) 1\left\{\frac{\sqrt{n}\left|\Delta_{i}^{n} Z\right|}{\sqrt{\hat{V}_{n, j}}}>\alpha n^{1 / 2-\varpi}\right\}, \\
& \chi_{n, i, j}(1,3)=\left(\frac{\sqrt{n} \Delta_{i}^{n} W}{\sigma_{\frac{(j-1) k_{n}}{n}}}\left(\sigma_{\frac{i-1}{n}}-\sigma_{\frac{(j-1) k_{n}}{n}}\right)-\xi_{n, i, j}(3)\right) 1\left\{\frac{\sqrt{n}\left|\Delta_{i}^{n} Z\right|}{\sqrt{\hat{V}_{n, j}}} \leq \alpha n^{1 / 2-\varpi}\right\} .
\end{aligned}
$$

- $\chi_{n, i, j}(2)=\left(\frac{\sqrt{\hat{V}_{n, j}(i)}}{\sigma_{(j-1) k_{n}}}-1-\xi_{n, j}(1)+\xi_{n, j}(2)\right)+\left(\xi_{n, j}(1)-\xi_{n, j}(2)-\tilde{\xi}_{n, i, j}(1)+\tilde{\xi}_{n, i, j}(2)\right)$. 


\section{A.3 Proof of Theorem 1}

The proof to follow can be divided in two main parts, one establishing the central limit theory for the leading terms and one establishing bounds for lower order terms. The latter follows along the same line as Todorov \& Tauchen (2014) and we refer to Section A.2 for definitions of corresponding terms. The central limit theory is established through a sequence of auxiliary lemmas in Section A.6. We shall make the references clear when necessary. Without loss of generality, we shall throughout assume that $\tau<0$ as well as $k_{n}-m_{n}>2$, which is no restriction since $m_{n} \ll k_{n}$. Now, let us start by making a decomposition

$$
\widehat{\mathcal{F}}_{W, n}^{*} \equiv \sqrt{N_{n}(\alpha, \varpi)}\left(\widehat{F}_{W, n}^{*}(\tau)-\widehat{F}_{n}(\tau)\right) \equiv \widehat{\mathcal{G}}_{n}^{*}(\tau)-\widehat{R}_{n}^{*}(\tau)
$$

where

$$
\begin{aligned}
& \widehat{\mathcal{G}}_{n}^{*}(\tau)=\frac{\sqrt{N_{n}(\alpha, \varpi)}}{\left\lfloor n / k_{n}\right\rfloor m_{n}} \sum_{j=1}^{\left\lfloor n / k_{n}\right\rfloor} \sum_{i=1}^{m_{n}}\left(X_{(j-1) k_{n}+i}-\Phi(\tau)\right) v_{(j-1) k_{n}+i}^{*}, \quad \text { and } \\
& \widehat{R}_{n}^{*}(\tau)=\left(\widetilde{F}_{n}(\tau)-\Phi(\tau)\right) \frac{\sqrt{N_{n}(\alpha, \varpi)}}{\left\lfloor n / k_{n}\right\rfloor m_{n}} \sum_{j=1}^{\left\lfloor n / k_{n}\right\rfloor} \sum_{i=1}^{m_{n}} v_{(j-1) k_{n}+i}^{*} .
\end{aligned}
$$

Since by Assumption DWB and Lemma 2 it readily follows that

$$
\sup _{\tau \in \mathcal{A}}\left|\widehat{R}_{n}^{*}(\tau)\right|=\sup _{\tau \in \mathcal{A}}\left|\widetilde{F}_{n}(\tau)-\Phi(\tau)\right| \times\left|\frac{\sqrt{N_{n}(\alpha, \varpi)}}{\left\lfloor n / k_{n}\right\rfloor m_{n}} \sum_{j=1}^{\left\lfloor n / k_{n}\right\rfloor} \sum_{i=1}^{m_{n}} v_{(j-1) k_{n}+i}^{*}\right| \leq O_{p}^{*}\left(\sqrt{\frac{m_{n}}{N_{n}(\alpha, \omega)} \frac{b_{n}}{m_{n}}}\right),
$$

in probability- $\mathbb{P}$, where, again, $\mathcal{A} \subset \mathbb{R} \backslash 0$ denotes a finite union of compact sets with positive Lebesgue measure, we can analyze the properties of $\widehat{\mathcal{G}}_{n}^{*}(\tau)$ rather than those of $\widehat{\mathcal{F}}_{W, n}^{*}$. Next, recall that the statistics $\widehat{\mathcal{G}}_{n}(\tau)$ and $\mathcal{G}_{n}(\tau)$ denote the bias-corrected empirical process and its asymptotic distribution, respectively, defined as in (16), then we will show that

$$
\sup _{x \in \mathbb{R}}\left|\mathbb{P}^{*}\left(\widehat{\mathcal{G}}_{n}^{*}(\tau) \leq x\right)-\mathbb{P}\left(\widehat{\mathcal{G}}_{n}(\tau) \leq x\right)\right| \stackrel{\mathbb{P}}{\rightarrow} 0,
$$

locally uniformly in $\tau \in \mathcal{A}$. Under the conditions for Lemma 2 , it follows $\widehat{\mathcal{G}}_{n}(\tau) \stackrel{d}{\rightarrow} \mathcal{G}_{n}(\tau)$, again, locally uniformly in $\tau$, by applying the central limit theory in the lemma in conjunction with Slutsky's Theorem since $N_{n}(\alpha, \varpi) /\left(\left\lfloor n / k_{n}\right\rfloor m_{n}\right) \stackrel{\mathbb{P}}{\rightarrow} 1$. Now, by utilizing this distribution result, we may invoke Polya's Theorem, see, e.g., Bhattacharya \& Rao (1986), to establish

$$
\sup _{x \in \mathbb{R}}\left|\mathbb{P}\left(\widehat{\mathcal{G}}_{n}(\tau) \leq x\right)-\mathbb{P}\left(\mathcal{G}_{n}(\tau) \leq x\right)\right| \stackrel{\mathbb{P}}{\rightarrow} 0 .
$$

Hence, if we can prove that

$$
\sup _{x \in \mathbb{R}}\left|\mathbb{P}\left(\widehat{\mathcal{G}}_{n}^{*}(\tau)\right)-\mathbb{P}\left(\mathcal{G}_{n}(\tau) \leq x\right)\right| \stackrel{\mathbb{P}}{\rightarrow} 0
$$


then (A.3) follows by the triangle inequality. To this end, let us introduce the two quantities

$$
\begin{aligned}
& \widehat{H}_{n, 1}^{*}(\tau) \equiv \frac{1}{\left\lfloor n / m_{n}\right\rfloor m_{n}} \sum_{j=1}^{\left\lfloor n / k_{n}\right\rfloor} \sum_{i=(j-1) k_{n}+1}^{(j-1) k_{n}+m_{n}}\left(1\left\{\sqrt{n} \Delta_{i}^{n} W \leq \tau\right\}-\Phi(\tau)\right) v_{i}^{*}, \\
& \widehat{H}_{n, 2}^{*}(\tau) \equiv \frac{\Phi^{\prime}(\tau) \tau}{\left\lfloor n / k_{n}\right\rfloor} \sum_{j=1}^{\left\lfloor n / k_{n}\right\rfloor} \zeta_{n, j} v_{(j-1) k_{n}+1}^{*}, \zeta_{n, j} \equiv \frac{1}{2}\left(\frac{\pi n}{2\left(k_{n}-1\right)} \sum_{i=(j-1) k_{n}+2}^{j k_{n}}\left|\Delta_{i-1}^{n} W \| \Delta_{i}^{n} W\right|-1\right),
\end{aligned}
$$

and make the decomposition $\widehat{\mathcal{G}}_{n}^{*}(\tau)=\widehat{\mathcal{G}}_{n, 1}^{*}(\tau)+\widehat{\mathcal{G}}_{n, 2}^{*}(\tau)$ where

$$
\begin{aligned}
& \widehat{\mathcal{G}}_{n, 1}^{*} \equiv \sqrt{N_{n}(\alpha, \varpi)}\left(\widehat{H}_{n, 1}^{*}(\tau)+\widehat{H}_{n, 2}^{*}(\tau)\right) \\
& \widehat{\mathcal{G}}_{n, 2}^{*} \equiv \frac{\sqrt{N_{n}(\alpha, \varpi)}}{\left\lfloor n / k_{n}\right\rfloor m_{n}} \sum_{j=1}^{\left\lfloor n / k_{n}\right\rfloor} \sum_{i=1}^{m_{n}}\left(X_{(j-1) k_{n}+i}-\Phi(\tau)\right) v_{(j-1) k_{n}+i}^{*}-\widehat{\mathcal{G}}_{n, 1}^{*} .
\end{aligned}
$$

The proof now proceeds in two steps:

Step 1: Show $\widehat{\mathcal{G}}_{n, 1}^{*}(\tau) \stackrel{d^{*}}{\rightarrow} \mathcal{G}(\tau)$ in probability-P , locally uniformly in $\tau$. Then, we may use the same arguments as for (A.4) to establish (A.5)

Step 2: Show $\left|\widehat{\mathcal{G}}_{n, 2}^{*}\right| \stackrel{\mathbb{P}^{*}}{\rightarrow} 0$ in probability- $\mathbb{P}$, locally uniformly in $\tau$.

First, for Step 1, define $\widetilde{\mathcal{G}}_{n, 1}^{*}=\sqrt{\left\lfloor n / k_{n}\right\rfloor m_{n} / N_{n}(\alpha, \varpi)} \widehat{\mathcal{G}}_{n, 1}^{*}$. Then, stated central limit theorem follows by invoking Lemma A.3 for $\widetilde{\mathcal{G}}_{n, 1}^{*}$ and applying this with $\left\lfloor n / k_{n}\right\rfloor m_{n} / N_{n}(\alpha, \varpi) \stackrel{\mathbb{P}}{\rightarrow} 1$, the continuous mapping theorem and Slutsky's theorem.

Next, for Step 2, write similarly $\widetilde{\mathcal{G}}_{n, 2}^{*}=\sqrt{\left\lfloor n / k_{n}\right\rfloor m_{n} / N_{n}(\alpha, \varpi)} \widehat{\mathcal{G}}_{n, 2}^{*}$ and further decompose the term as $\widetilde{\mathcal{G}}_{n, 2}^{*}=\widetilde{\mathcal{G}}_{n, 2,1}^{*}-\widetilde{\mathcal{G}}_{n, 2,2}^{*}-\widetilde{\mathcal{G}}_{n, 1}^{*}$, with $\widetilde{\mathcal{G}}_{n, 1}^{*}$ being defined as in Step $\mathbf{1}$ and where

$$
\begin{aligned}
& \widetilde{T}_{n, 1}^{*}(\tau) \equiv \frac{1}{\sqrt{\left\lfloor n / k_{n}\right\rfloor m_{n}}} \widetilde{\mathcal{G}}_{n, 2,1}^{*} \equiv \frac{1}{\left\lfloor n / k_{n}\right\rfloor m_{n}} \sum_{j=1}^{\left\lfloor n / k_{n}\right\rfloor} \sum_{i=1}^{m_{n}} X_{(j-1) k_{n}+i} v_{(j-1) k_{n}+i}^{*}, \\
& \widetilde{T}_{n, 2}^{*}(\tau) \equiv \frac{1}{\sqrt{\left\lfloor n / k_{n}\right\rfloor m_{n}}} \widetilde{\mathcal{G}}_{n, 2,2}^{*} \equiv \frac{1}{\left\lfloor n / k_{n}\right\rfloor m_{n}} \sum_{j=1}^{\left\lfloor n / k_{n}\right\rfloor} \sum_{i=1}^{m_{n}} \Phi(\tau) v_{(j-1) k_{n}+i}^{*} .
\end{aligned}
$$

Now, let us define

$$
\begin{array}{r}
\widehat{T}_{n}^{*}(\tau) \equiv \frac{1}{\left\lfloor n / k_{n}\right\rfloor m_{n}} \sum_{j=1}^{\left\lfloor n / k_{n}\right\rfloor} \sum_{i=(j-1) k_{n}+1}^{(j-1) k_{n}+m_{n}} 1\left\{\sqrt{n} \frac{\Delta_{i}^{n} Z}{\sigma_{(j-1) k_{n}}} 1\left\{\frac{\left|\Delta_{i}^{n} Z\right|}{\sqrt{\hat{V}_{n, j}}} \leq \alpha n^{-\varpi}\right\}\right. \\
\left.\leq \tau \frac{\sqrt{\hat{V}_{n, j}(i)}}{\sigma_{\frac{(j-1) k_{n}}{n}}^{n}}-\chi_{n, i, j(1)}-\tau \chi_{n, i, j}(2)\right\} v_{i}^{*}
\end{array}
$$




$$
=\frac{1}{\left\lfloor n / k_{n}\right\rfloor m_{n}} \sum_{j=1}^{\left\lfloor n / k_{n}\right\rfloor} \sum_{i=(j-1) k_{n}+1}^{(j-1) k_{n}+m_{n}} 1\left\{\sqrt{n} \Delta_{i}^{n} W \leq \tau+\tau \tilde{\xi}_{n, i, j}(1)-\tau \tilde{\xi}_{n, i, j}(2)-\tilde{\xi}_{n, i, j}(3)\right\} v_{i}^{*}
$$

by applying the definitions in Section A.2. We now show that $k_{n}\left(\widetilde{T}_{n, 1}^{*}(\tau)-\widehat{T}_{n}^{*}(\tau)\right)=o_{p}^{*}(1)$, in probability- $\mathbb{P}$, such that we may work with $\widehat{T}_{n}^{*}(\tau)$ in the remainder of the proof. To this end, write

$$
\begin{aligned}
\mathbb{E}^{*}\left[\left|\widetilde{T}_{n, 1}^{*}(\tau)-\widehat{T}_{n}^{*}(\tau)\right|\right] & \leq \frac{1}{\left\lfloor n / k_{n}\right\rfloor m_{n}} \sum_{j=1}^{\left\lfloor n / k_{n}\right\rfloor} \sum_{i=(j-1) k_{n}+1}^{(j-1) k_{n}+m_{n}} \mathbb{E}\left|v_{i}^{*}\right| \\
& \times\left|X_{i}-1\left\{\sqrt{n} \Delta_{i}^{n} W \leq \tau+\tau \tilde{\xi}_{n, i, j}(1)-\tau \tilde{\xi}_{n, i, j}(2)-\tilde{\xi}_{n, i, j}(3)\right\}\right|
\end{aligned}
$$

Next, let $\eta_{n}$ be a sequence of positive numbers that only depend on $n$, then we may use the fact that the probability density of standard normal random variable is uniformly bounded to write

$$
\begin{aligned}
& \mathbb{E}\left|X_{i}-1\left\{\sqrt{n} \Delta_{i}^{n} W \leq \tau+\tau \tilde{\xi}_{n, i, j}(1)-\tau \tilde{\xi}_{n, i, j}(2)-\tilde{\xi}_{n, i, j}(3)\right\}\right| \leq \mathbb{P}\left(\left(\left|\chi_{n, i, j}(1)\right|+\left|\chi_{n, i, j}(2)\right|\right)>\eta_{n}\right) \\
& +\mathbb{E}\left|\Phi\left(\frac{\tau+\tau \tilde{\xi}_{n, i, j}(1)-\tau \tilde{\xi}_{n, i, j}(2)+\eta_{n}(1+|\tau|)}{\tilde{\xi}_{n, i, j}(4)}\right)-\Phi\left(\frac{\tau+\tau \tilde{\xi}_{n, i, j}(1)-\tau \tilde{\xi}_{n, i, j}(2)-\eta_{n}(1+|\tau|)}{\tilde{\xi}_{n, i, j}(4)}\right)\right| \\
& \leq K\left(\mathbb{P}\left(\left(\left|\chi_{n, i, j}(1)\right|+\left|\chi_{n, i, j}(2)\right|\right)>\eta_{n}\right)+\eta_{n}|\tau|\right),
\end{aligned}
$$

similarly to the corresponding term in Todorov \& Tauchen (2014, Section 10.4.1). Hence, we may invoke their bounds in equations (10.14), (10.16)-(10.19), (10.28), (10.29) and (10.31) to show

$$
\mathbb{P}\left(\left(\left|\chi_{n, i, j}(1)\right|+\left|\chi_{n, i, j}(2)\right|\right)>\eta_{n}\right) \leq K\left[\frac{1}{n \eta_{n}} \bigvee \frac{1}{\eta_{n}^{p}\left[n^{p / 2} \wedge\left(n / k_{n}\right)^{p} \wedge k_{n}^{3 p / 2}\right]} \bigvee\left(\frac{k_{n}}{n}\right)^{\frac{1}{1+\iota}} \frac{1}{\eta_{n}^{\iota}}\right],
$$

for every $p \geq 1$ and arbitrarily small $\iota>0$. Hence, by picking $\eta_{n} \asymp n^{-q-\iota}, \iota \in(0,1 / 2-q)$ and combining (A.6) with Assumption DWB and (A.7), $k_{n} \mathbb{E}^{*}\left[\left|\widetilde{T}_{n, 1}^{*}(\tau)-\widehat{T}_{n}^{*}(\tau)\right|\right] \leq o_{p}^{*}(1)$ such that for any compact subset, $\mathcal{A}$, we have

$$
\sup _{\tau \in \mathcal{A}}\left|\widetilde{T}_{n, 1}^{*}(\tau)-\widehat{T}_{n}^{*}(\tau)\right|=o_{p}^{*}\left(1 / k_{n}\right) .
$$

Now, let us make the decomposition,

$$
\widehat{T}_{n}^{*}(\tau)-\widetilde{T}_{n, 2}^{*}(\tau)=\sum_{i=1}^{6} A_{n, i}^{*}
$$

where $A_{n, 1}^{*}=\widehat{H}_{n, 1}^{*}(\tau)$ and the remaining terms are defined as

$$
A_{n, 2}^{*}=\frac{1}{\left\lfloor n / k_{n}\right\rfloor} \sum_{j=1}^{\left\lfloor n / k_{n}\right\rfloor}\left(\Phi\left(\tau+\tau \bar{\xi}_{n, j}(1)-\tau \bar{\xi}_{n, j}(2)\right)-\Phi(\tau)\right) v_{(j-1) k_{n}+1}^{*},
$$




$$
\begin{aligned}
& A_{n, 3}^{*}=\frac{1}{\left\lfloor n / k_{n}\right\rfloor m_{n}} \sum_{j=1}^{\left\lfloor n / k_{n}\right\rfloor} \sum_{i=(j-1)+1}^{(j-1) k_{n}+m_{n}} a_{n, i} \times v_{i}^{*}, \quad \text { where } \\
& a_{n, i}=1\left\{\sqrt{n} \Delta_{i}^{n} W \leq \frac{\tau+\tau \tilde{\xi}_{n, i, j}(1)-\tau \tilde{\xi}_{n, i, j}(2)}{\xi_{n, i, j}(4)}\right\}-1\left\{\sqrt{n} \Delta_{i}^{n} W \leq \tau\right\} \\
& +\Phi(\tau)-\Phi\left(\frac{\tau+\tau \tilde{\xi}_{n, i, j}(1)-\tau \tilde{\xi}_{n, i, j}(2)}{\xi_{n, i, j}(4)}\right) \\
& A_{n, 4}^{*}=\frac{1}{\left\lfloor n / k_{n}\right\rfloor m_{n}} \sum_{j=1}^{\left\lfloor n / k_{n}\right\rfloor} \sum_{i=(j-1) k_{n}+1}^{(j-1) k_{n}+m_{n}} v_{i}^{*} \\
& \times\left[\Phi\left(\tau+\tau \tilde{\xi}_{n, i, j}(1)-\tau \tilde{\xi}_{n, i, j}(2)\right)-\Phi\left(\tau+\tau \bar{\xi}_{n, j}(1)-\tau \bar{\xi}_{n, j}(2)\right)\right], \\
& A_{n, 5}^{*}=\frac{1}{\left\lfloor n / k_{n}\right\rfloor m_{n}} \sum_{j=1}^{\left\lfloor n / k_{n}\right\rfloor} \sum_{i=(j-1) k_{n}+1}^{(j-1) k_{n}+m_{n}} v_{i}^{*} \\
& \times\left[\Phi\left(\frac{\tau+\tau \tilde{\xi}_{n, i, j}(1)-\tau \tilde{\xi}_{n, i, j}(2)}{\xi_{n, i, j}(4)}\right)-\Phi\left(\tau+\tau \tilde{\xi}_{n, i, j}(1)-\tau \tilde{\xi}_{n, i, j}(2)\right)\right], \\
& A_{n, 6}^{*}=\frac{1}{\left\lfloor n / k_{n}\right\rfloor m_{n}} \sum_{j=1}^{\left\lfloor n / k_{n}\right\rfloor} \sum_{i=(j-1)+1}^{(j-1) k_{n}+m_{n}}\left(\Phi\left(\tau+\tau \bar{\xi}_{n, j}(1)-\tau \bar{\xi}_{n, j}(2)\right)-\Phi(\tau)\right) v_{i}^{*}-A_{n, 2}^{*} .
\end{aligned}
$$

Hence, we need to establish bounds for $A_{n, 2}^{*}-\widehat{H}_{n, 2}^{*}(\tau), A_{n, 3}^{*}, A_{n, 4}^{*}, A_{n, 5}^{*}$ and $A_{n, 6}^{*}$. For the first of these terms, apply a second-order Taylor expansion for $A_{n, 2}^{*}$ to obtain the leading terms,

$$
\begin{aligned}
& A_{n, 2}^{*}(1)=\frac{1}{\left\lfloor n / k_{n}\right\rfloor} \sum_{j=1}^{\left\lfloor n / k_{n}\right\rfloor} \Phi^{\prime}(\tau) \tau \bar{\xi}_{n, j}(1) v_{(j-1) k_{n}+1}^{*} \\
& A_{n, 2}^{*}(2)=\frac{1}{\left\lfloor n / k_{n}\right\rfloor} \sum_{j=1}^{\left\lfloor n / k_{n}\right\rfloor}\left(\frac{\Phi^{\prime \prime}(\tau) \tau^{2}\left(\bar{\xi}_{n, j}(1)\right)^{2}}{2}-\Phi^{\prime}(\tau) \tau \bar{\xi}_{n, j}(2)\right) v_{(j-1) k_{n}+1}^{*} .
\end{aligned}
$$

Then, by using the bounds

$$
\left\{\begin{array}{l}
\mathbb{E}\left|\bar{V}_{n, j}-\sigma_{\frac{(j-1) k_{n}}{n}}^{2}\right|^{p} \leq K k_{n}^{-p / 2} \\
\mathbb{E}_{(j-1) k_{n}}^{n}\left[R_{j}^{(4)}\right]=0, \quad \mathbb{E}\left|R_{j}^{(4)}\right|^{p} \leq K\left(k_{n} / n\right)^{p / 2}, \quad \forall p \geq 2, \\
\mathbb{E}\left[\left|R_{j}^{(4)}-\widetilde{R}_{i, j}^{(4)}\right|^{p}+\left|\widetilde{R}_{i, j}^{(4)}-\widetilde{R}_{i, j}^{(4)}(i)\right|^{p}\right] \leq K\left(\frac{1}{\sqrt{n}}\right)^{p}, \quad \forall p>0,
\end{array}\right.
$$

cf. Todorov \& Tauchen (2014, Equations (10.25)-(10.26)), in conjunction with Assumption DWB an the fact the probability density of a standard normal density and $\Phi^{\prime \prime}$ are uniformly bounded, we have

$$
\mathbb{E}\left[\mathbb{E}^{*}\left|A_{n, 2}^{*}-A_{n, 2}^{*}(1)-A_{n, 2}^{*}(2)\right|\right] \leq K\left(|\tau|^{3} \vee|\tau|^{2}\right)\left[\left(\frac{k_{n}}{n}\right)^{3 / 2} \bigvee\left(\frac{1}{k_{n}}\right)^{3 / 2}\right]
$$


and, as a result, $\sup _{\tau \in \mathcal{A}}\left|A_{n, 2}^{*}-A_{n, 2}^{*}(1)-A_{n, 2}^{*}(2)\right|=o_{p}^{*}\left(1 / k_{n}\right)$ in probability- $\mathbb{P}$, similarly to (A.8). For the first of the two Taylor expansion terms, write

$$
\mathbb{E}^{*}\left|A_{n, 2}^{*}(1)-\widehat{H}_{n, 2}^{*}(\tau)\right| \leq \frac{1}{\left\lfloor n / k_{n}\right\rfloor} \sum_{j=1}^{\left\lfloor n / k_{n}\right\rfloor}\left|\Phi^{\prime}(\tau) \tau\right| \times\left|\bar{\xi}_{n, j}(1)-\zeta_{n, j}\right| \times \mathbb{E}\left|v_{(j-1) k_{n}+1}^{*}\right| .
$$

Hence, by Assumption DWB and the bounds in (A.10), we have

$$
\mathbb{E}\left|\bar{\xi}_{n, j}(1)-\zeta_{n, j}\right| \leq K|\tau|\left(\frac{1}{\sqrt{n}} \bigvee \frac{k_{n}}{n}\right)
$$

and, consequently, it follows that $\left|A_{n, 2}^{*}(1)-\widehat{H}_{n, 2}^{*}(\tau)\right| \leq o_{p}^{*}\left(1 / k_{n}\right)$, in probability- $\mathbb{P}$, locally uniformly in the argument $\tau$. For the second Taylor expansion term, $A_{n, 2}^{*}(2)$, make the decomposition

$$
\begin{aligned}
A_{n, 2}^{*}(2) & =A_{n, 2}^{*}(2,1)+A_{n, 2}^{*}(2,2), \quad A_{n, 2}^{*}(2,1)=\frac{H_{3}(\tau)}{\left\lfloor n / k_{n}\right\rfloor k_{n}} \sum_{j=1}^{\left\lfloor n / k_{n}\right\rfloor} v_{(j-1) k_{n}+1}^{*}, \\
A_{n, 2}^{*}(2,2) & =\frac{1}{\left\lfloor n / k_{n}\right\rfloor} \sum_{j=1}^{\left\lfloor n / k_{n}\right\rfloor}\left(\frac{\Phi^{\prime \prime}(\tau) \tau^{2}\left(\bar{\xi}_{n, j}(1)\right)^{2}}{2}-\Phi^{\prime}(\tau) \tau \bar{\xi}_{n, j}(2)-H_{3}(\tau) / k_{n}\right) v_{(j-1) k_{n}+1}^{*} .
\end{aligned}
$$

For the first of these terms, we have

$$
\left|A_{n, 2}^{*}(2,1)\right| \leq K\left(|\tau| \vee \tau^{2}\right) \times O_{p}\left(\sqrt{b_{n} / m_{n}} /\left(\sqrt{k_{n} n}\right)\right) \leq K\left(|\tau| \vee \tau^{2}\right) \times O_{p}\left(1 /\left(\sqrt{k_{n} n}\right)\right)
$$

by computing the mean and variance using Assumption DWB. ${ }^{16}$ For the second term,

$$
\mathbb{E}^{*}\left|A_{n, 2}^{*}(2,2)\right| \leq \frac{1}{\left\lfloor n / k_{n}\right\rfloor} \sum_{j=1}^{\left\lfloor n / k_{n}\right\rfloor}\left|\frac{\Phi^{\prime \prime}(\tau) \tau^{2}\left(\bar{\xi}_{n, j}(1)\right)^{2}}{2}-\Phi^{\prime}(\tau) \tau \bar{\xi}_{n, j}(2)-H_{3}(\tau) / k_{n}\right| \times \mathbb{E}\left|v_{(j-1) k_{n}+1}^{*}\right| .
$$

As in (A.12), we may apply (A.10) to show $\mathbb{E}\left|\bar{\xi}_{n, j}(2)-\hat{\xi}_{n, j}(2)\right| \leq K\left(|\tau| \vee \tau^{2}\right)\left(n^{-1 / 2} \vee\left(k_{n} / n\right)\right)$. Moreover, since we have $\left(\bar{\xi}_{n, j}(1)\right)^{2} / 2=\bar{\xi}_{n, j}(2)$ as well as

$$
\mathbb{E}_{(j-1) k_{n}}^{n}\left[\hat{\xi}_{n, j}(2)\right]=\frac{1}{8 k_{n}}\left(\left(\frac{\pi}{2}\right)^{2}+\pi-3\right)+o\left(1 / k_{n}\right),
$$

we may collect bounds, Assumption DWB and successive conditioning to show

$$
\left|A_{n, 2}^{*}(2,2)\right| \leq K\left(|\tau| \vee \tau^{2}\right) O_{p}^{*}\left(\frac{1}{\sqrt{n}} \bigvee \frac{k_{n}}{n}\right)+o_{p}^{*}\left(1 / k_{n}\right),
$$

\footnotetext{
${ }^{16} \overline{\text { Too see this, note that } \mathbb{E}\left[A_{n, 2}^{*}(2,1)\right]=0 \text { and } \operatorname{Cov}}\left(v_{r}^{*}, v_{s}^{*}\right)=O_{p}\left(b_{n} /\left(m_{n}\left\lfloor n / k_{n}\right\rfloor\right)\right)$ for integers $r$ and $s$ by Assumption DWB. Moreover, since the scale is $1 /\left(\left\lfloor n / k_{n}\right\rfloor k_{n}\right)^{2}$ for the second moment and $m_{n} / k_{n} \rightarrow 0$, the bound follows immediately.
} 
in probability- $\mathbb{P}$, locally uniformly in $\tau$. Hence, by combining results,

$$
\left|A_{n, 2}^{*}-\widehat{H}_{n, 2}^{*}(\tau)\right|=o_{p}^{*}\left(1 / k_{n}\right)
$$

in probability- $\mathbb{P}$, locally uniformly in $\tau .{ }^{17}$

For the next term, $A_{n, 3}^{*}$, we readily have $\mathbb{E}^{*}\left[A_{n, 3}^{*}\right]=0$ by $\mathbb{E}^{*}\left[a_{n, i} v_{i}^{*}\right]=a_{n, i} \mathbb{E}\left[v_{i}^{*}\right]=0$. Moreover, we have $\mathbb{E}\left[a_{n, i}\right]=0, \mathbb{E}\left[a_{n, i}^{2}\right] \leq K|\tau|\left(\left(k_{n} / n\right)^{1 / 2} \vee k_{n}^{-1 / 2}\right)$, and $\mathbb{E}\left[a_{n, i} a_{n, g}\right]=0$ for $|i-g|>k_{n}$ due to independence of the Brownian increments $\Delta_{i}^{n} W, \Delta_{g}^{n} W, \Delta_{i}^{n} W^{\prime}$ and $\Delta_{g}^{n} W^{\prime}$. When $|i-g| \leq k_{n}$, we follow Todorov \& Tauchen (2014) and use the fact that $\xi_{n, i, j}(4)$ is adapted to $\mathcal{F}_{t_{i-1}}$ as well as decompose the $a_{n, g}$ component into a part with the $i$ th increment removed from $\tilde{\xi}_{n, g, j}(1)$ and $\tilde{\xi}_{n, g, j}(2)$, denoted by $\bar{a}_{n, g}$, and a residual $\tilde{a}_{n, g}=a_{n, g}-\bar{a}_{n, g}$. For these terms, we have $\mathbb{E}\left[a_{n, g} \bar{a}_{n, g}\right]=0$ and, by their arguments (cf. pp. 1880-1881), the triangle inequality and Chebyshev's inequality,

$$
\mathbb{E}\left|a_{n, g} \tilde{a}_{n, g}\right| \leq K\left(|\tau| \vee \tau^{2}\right)\left(\left(\frac{k_{n}}{n}\right)^{1-2 \iota} \bigvee \frac{1}{k_{n}^{1-2 \iota}}\right)
$$

for some arbitrarily small $\iota>0$ and sufficiently large $n$. We apply these results in conjunction with the convergence part of Assumption DWB, $\operatorname{Cov}\left(v_{i}^{*}, v_{g}^{*}\right) \rightarrow C_{i, g}$ for all $(i, g) \in 1, \ldots n$ where $C_{i, g} \geq 0$ is a nonrandom constant, the triangle inequality as well as the Cauchy-Schwarz inequality to show

$$
\begin{aligned}
\mathbb{E}^{*}\left[\left(A_{n, 3}^{*}\right)^{2}\right] & =\frac{1}{\left(\left\lfloor n / k_{n}\right\rfloor m_{n}\right)^{2}} \sum_{j=1}^{\left\lfloor n / k_{n}\right\rfloor} \sum_{i=(j-1) k_{n}+1}^{(j-1)+m_{n}} \sum_{h=1}^{\left\lfloor n / k_{n}\right\rfloor} \sum_{g=(j-1) k_{n}+1}^{(j-1)+m_{n}} a_{n, i} a_{n, g} \operatorname{Cov}\left(v_{i}^{*}, v_{g}^{*}\right) \\
& \leq \frac{K\left(|\tau| \vee \tau^{2}\right)}{\left(\left\lfloor n / k_{n}\right\rfloor m_{n}\right)^{2}} \sum_{j=1}^{\left\lfloor n / k_{n}\right\rfloor} O_{p}\left(m_{n}\left(\sqrt{\frac{k_{n}}{n}} \bigvee \frac{1}{\sqrt{k_{n}}}\right)+m_{n}^{2}\left(\left(\frac{k_{n}}{n}\right)^{1-2 \iota} \bigvee \frac{1}{k_{n}^{1-2 \iota}}\right)\right) \\
& =K\left(|\tau| \vee \tau^{2}\right) \times O_{p}\left(\frac{1}{\left\lfloor n / k_{n}\right\rfloor m_{n}}\left(\frac{1}{k_{n}}\right)^{1 / 2} \bigvee \frac{k_{n}^{2 \iota}}{n}\right),
\end{aligned}
$$

which, consequently, provides the bound $\left|A_{n, 3}^{*}\right| \leq o_{p}^{*}\left(1 / k_{n}\right)$ in probability- $\mathbb{P}$, locally uniformly in $\tau$.

Next, for $A_{n, 4}^{*}$, write

$$
\begin{aligned}
\mathbb{E}^{*}\left[\left|A_{n, 4}^{*}\right|\right] & \leq \frac{1}{\left\lfloor n / k_{n}\right\rfloor m_{n}} \sum_{j=1}^{\left\lfloor n / k_{n}\right\rfloor} \sum_{(j-1) k_{n}+1}^{(j-1) k_{n}+m_{n}}\left|\widehat{A}_{n, 4, i}\right| \times \mathbb{E}\left|v_{i}^{*}\right|, \quad \text { where } \\
\widehat{A}_{n, 4, i} & \equiv \Phi\left(\tau+\tau \tilde{\xi}_{n, i, j}(1)-\tau \tilde{\xi}_{n, i, j}(2)\right)-\Phi\left(\tau+\tau \bar{\xi}_{n, j}(1)-\tau \bar{\xi}_{n, j}(2)\right) .
\end{aligned}
$$

Then, we make a Taylor expansion, similarly to the one for $A_{n, 2}^{*}$, and use the same arguments as in (A.11), (A.12) and for $A_{n, 2}^{*}$ to show $\left|\widehat{A}_{n, 4, i}\right| \leq K\left(|\tau| \vee \tau^{2}\right) O_{p}\left(n^{-1 / 2} \vee\left(k_{n} / n\right)\right)+o_{p}\left(1 / k_{n}\right)$. By combining this with Assumption DWB, we have $\left|A_{n, 4}^{*}\right| \leq o_{p}^{*}\left(1 / k_{n}\right)$ in probability- $\mathbb{P}$, locally uniformly in $\tau$.

\footnotetext{
${ }^{17}$ This shows that the remaining asymptotic bias is negligible for the local DWB, that is, the statistic is bias-corrected.
} 
For $A_{n, 5}^{*}$, we make a decomposition similarly to Todorov \& Tauchen (2014, pp. 1881-1882). Hence, by the triangle inequality, $\forall \iota>0$ and $n$ sufficiently high, write

$$
\mathbb{E}^{*}\left[\left|A_{n, 5}^{*}\right|\right] \leq \frac{1}{\left\lfloor n / k_{n}\right\rfloor m_{n}} \sum_{j=1}^{\left\lfloor n / k_{n}\right\rfloor} \sum_{(j-1) k_{n}+1}^{(j-1) k_{n}+m_{n}}\left|b_{n, i}(1)+b_{n, i}(2)+b_{n, i}(3)\right| \times \mathbb{E}\left|v_{i}^{*}\right|,
$$

where $b_{n, i}(1), b_{n, i}(2)$, and $b_{n, i}(3)$ are defined as

$$
\begin{gathered}
b_{n, i}(1) \equiv\left[\Phi\left(\frac{\tau+\tau \tilde{\xi}_{n, i, j}(1)-\tau \tilde{\xi}_{n, i, j}(2)}{\xi_{n, i, j}(4)}\right)-\Phi\left(\tau+\tau \tilde{\xi}_{n, i, j}(1)-\tau \tilde{\xi}_{n, i, j}(2)\right)\right] \\
\times 1\left\{\left|\xi_{n, i, j}(4)-1\right| \geq\left(k_{n} / n\right)^{1 / 2-\iota}\right\} \\
b_{n, i}(2) \equiv \Phi^{\prime}\left(\tau+\tau \tilde{\xi}_{n, i, j}(1)-\tau \tilde{\xi}_{n, i, j}(1)\right)\left(\tau+\tau \tilde{\xi}_{n, i, j}(1)-\tau \tilde{\xi}_{n, i, j}(1)\right)\left(\xi_{n, i, j}(4)-1\right) \\
\times 1\left\{\left|\xi_{n, i, j}(4)-1\right|<\left(k_{n} / n\right)^{1 / 2-\iota}\right\}, \\
b_{n, i}(3) \leq K \frac{\left|\tau+\tau \tilde{\xi}_{n, i, j}(1)-\tau \tilde{\xi}_{n, i, j}(1)\right|^{2}}{\left(1-\left(k_{n} / n\right)^{1 / 2-\iota}\right)^{3}}\left|\xi_{n, i, j}(4)-1\right|^{2},
\end{gathered}
$$

for an arbitrarily small $\iota>0$. Moreover, we may readily invoke the following inequalities,

$$
\begin{aligned}
& \mathbb{E}\left[\left|b_{n, i}(1)\right|+\left|b_{n, i}(3)\right|\right] \leq K\left(\tau^{2} \vee 1\right) \frac{k_{n}}{n} \\
& \mathbb{E}\left|b_{n, i}(2)-\Phi^{\prime}(\tau) \tau\left(\xi_{n, i, j}(4)-1\right) 1\left\{\left|\xi_{n, i, j}(4)-1\right|<\left(k_{n} / n\right)^{1 / 2-\iota}\right\}\right| \leq K|\tau| \frac{1}{\sqrt{n}} .
\end{aligned}
$$

By combining these with Assumption DWB and the uniform boundedness of probability density of a standard normal distribution and its derivative, $\mathbb{E}^{*}\left[\left|A_{n, 5}^{*}\right|\right] \leq K\left(|\tau| \vee \tau^{2}\right) O_{p}\left(\left(k_{n} / n\right) \vee n^{-1 / 2}\right)$ such that it follows that $\left|A_{n, 5}^{*}\right| \leq o_{p}^{*}\left(1 / k_{n}\right)$ in probability- $\mathbb{P}$, locally uniformly in $\tau$.

For the last term, $A_{n, 6}^{*}$, define $B_{j}(\tau) \equiv \Phi\left(\tau+\tau \bar{\xi}_{n, j}(1)-\tau \bar{\xi}_{n, j}(2)\right)-\Phi(\tau)$ and rewrite the term as

$$
A_{n, 6}^{*}=\frac{1}{\left\lfloor n / k_{n}\right\rfloor} \sum_{j=1}^{\left\lfloor n / k_{n}\right\rfloor} B_{j}(\tau) z_{(j-1) k_{n}+1}^{*}, \quad \text { where } \quad z_{(j-1) k_{n}+1}^{*}=\frac{1}{m_{n}} \sum_{i=(j-1) k_{n}+2}^{(j-1) k_{n}+m_{n}} v_{i}^{*}
$$

Hence, $A_{n, 6}^{*}$ has the same form as $A_{n, 2}^{*}$ with $z_{(j-1) k_{n}+1}^{*}$ in place of $v_{(j-1) k_{n}+1}^{*}$ and may be treated in a similar manner. As a result, and analogously to the leading term $\widehat{H}_{n, 2}^{*}(\tau)$, define

$$
\widetilde{H}_{n, 2}^{*}(\tau) \equiv \frac{\Phi^{\prime}(\tau) \tau}{\left\lfloor n / k_{n}\right\rfloor} \sum_{j=1}^{\left\lfloor n / k_{n}\right\rfloor} \zeta_{n, j} z_{(j-1) k_{n}+1}^{*}
$$

then it follows by the same arguments provided for (A.14) that $\left|A_{n, 6}^{*}-\widetilde{H}_{n, 2}^{*}(\tau)\right|=o_{p}\left(1 / k_{n}\right)$, in probability- $\mathbb{P}$, locally uniformly in $\tau$. Next, $\mathbb{E}^{*}\left[\widetilde{H}_{n, 2}^{*}(\tau)\right]=0$ follows by Assumption DWB and, by 
additionally using boundedness of the probability density of a standard normal density as well independence of the Brownian increments, we have

$$
\mathbb{E}\left(\mathbb{E}^{*}\left[\left(\widetilde{H}_{n, 2}^{*}(\tau)\right)^{2}\right]\right) \leq \frac{K^{2} \tau^{2}}{\left(\left\lfloor n / k_{n}\right\rfloor\right)^{2}} \sum_{j=1}^{\left\lfloor n / k_{n}\right\rfloor} \mathbb{E}\left[\zeta_{n, j}^{2}\right] \times \mathbb{E}\left[\left(z_{(j-1) k_{n}+1}^{*}\right)^{2}\right]
$$

for which $\mathbb{E}\left[\zeta_{n, j}^{2}\right] \leq K$ by Lemma A.1(a) and with

$$
\mathbb{E}\left[\left(z_{(j-1) k_{n}+1}^{*}\right)^{2}\right]=\frac{1}{m_{n}^{2}} \sum_{i=(j-1) k_{n}+2}^{(j-1) k_{n}+m_{n}} \sum_{h=(j-1) k_{n}+2}^{(j-1) k_{n}+m_{n}} \operatorname{Cov}\left(v_{i}^{*}, v_{h}^{*}\right) \leq O\left(\frac{b_{n}}{\left\lfloor n / k_{n}\right\rfloor m_{n}}\right) .
$$

Hence, by combining results, $\left|\widetilde{H}_{n, 2}^{*}(\tau)\right| \leq O_{p}\left(\left(k_{n} / n\right) \sqrt{b_{n} / m_{n}}\right)=o_{p}^{*}\left(1 / k_{n}\right)$, in probability $\mathbb{P}$, locally uniformly in $\tau$, which, together with the triangle inequality, establishes that $\left|A_{n, 6}^{*}\right|=o_{p}^{*}\left(1 / k_{n}\right)$. Now, by collecting asymptotic bounds for the sequence $\sum_{i=1}^{6} A_{n, i}^{*}$ and using them in conjunction with (A.8), this shows $\left|\widetilde{\mathcal{G}}_{n, 2}^{*}\right| \leq o_{p}^{*}\left(\sqrt{\left(n m_{n}\right) / k_{n}^{3}}\right)=o_{p}^{*}(1)$, in probability-P , locally uniformly in $\tau$. Then, since we have $N_{n}(\alpha, \varpi) /\left(\left\lfloor n / k_{n}\right\rfloor m_{n}\right) \stackrel{\mathbb{P}}{\rightarrow} 1$, the final asymptotic bound for $\widehat{\mathcal{G}}_{n, 2}^{*}$ in Step 2 follows by an application of the continuous mapping theorem.

\section{A.4 Proof of Lemma 3}

Apart from the fourth moment result, then (a)-(c) follows by the properties of multinomial random variables and by $v_{(j-1) k_{n}+i}$ being constant across $i=1, \ldots, m_{n}$ for a given $j=1, \ldots,\left\lfloor n / k_{n}\right\rfloor$. For the fourth moment bound, we may use the $c_{r}$-inequality to deduce

$$
\mathbb{E}\left[\left|v_{i}^{\diamond}\right|^{4}\right] \leq K_{1} \mathbb{E}\left[\left|\zeta_{i}^{\diamond}\right|^{4}\right]+K_{2}<K
$$

for constants $K_{1}<\infty$ and $K_{2}<\infty$, using also the bound $\mathbb{E}\left[\left|\zeta_{i}^{\diamond}\right|^{4}\right]<15$, see, e.g., Præstgaard \& Wellner (1993, Example 3.2), for the last inequality. Finally, for (d), write

$$
\begin{aligned}
\sum_{i=1}^{\left\lfloor n / k_{n}\right\rfloor m_{n}} \operatorname{Cov}\left(u_{1}^{\diamond}, u_{i}^{\diamond}\right) & =\sum_{i=1}^{m_{n}} \operatorname{Cov}\left(u_{1}^{\diamond}, u_{i}^{\diamond}\right)+\sum_{i=m_{n}+1}^{\left\lfloor n / k_{n}\right\rfloor m_{n}} \operatorname{Cov}\left(u_{1}^{\diamond}, u_{i}^{\diamond}\right) \\
& =m_{n}\left(1-1 / p_{n}\right)-m_{n}\left(p_{n}-1\right) / p_{n}=o\left(m_{n}\right),
\end{aligned}
$$

using the variance-covariance properties (a)-(c), thus concluding the proof.

\section{A.5 Proof of Theorem 3}

The result, similarly to Doukhan et al. (2015, Theorem 4.4), follows by Beutner \& Zähle (2014, Theorem 3.14(ii)), if we can verify conditions (a)-(c) for the latter. First, for (a), we need to verify 
the conditions for their Lemmas 3.4 and 3.6. ${ }^{18}$ Specifically, conditions (a)-(c) of Lemmas 3.4 and 3.6 is satisfied since $\widehat{F}_{n}(\tau)$ is the empirical CDF, $\Phi(\tau)$ is Gaussian, $\tau_{1}, \tau_{2} \in \mathcal{A}$ and by the regularity conditions on the kernel function in Assumption 3. Next, for condition (b) of Beutner \& Zähle (2014, Theorem 3.14(ii)), this follows by Lemma 2, Assumption 3 and Beutner \& Zähle (2014, Remark 3.16). Finally, condition (c) follows by the locally uniform central limit theorem for the empirical process at high frequencies in Lemma 2, and since the limiting distribution, $\mathcal{G}_{n}(\tau)$, has continuous paths.

\section{A.6 Technical Results}

Lemma A.1 (Todorov \& Tauchen (2014), central limit theory for leading terms.). Suppose that the regularity conditions of Lemma 2 hold. Moreover, let

$$
\left(\begin{array}{c}
\sqrt{\left\lfloor n / k_{n}\right\rfloor m_{n}} \widehat{H}_{n, 1}(\tau) \\
\sqrt{\left\lfloor n / k_{n}\right\rfloor k_{n}} \widehat{H}_{n, 2}(\tau)
\end{array}\right) \equiv \sum_{i=1}^{\left\lfloor n / k_{n}\right\rfloor k_{n}}\left(\begin{array}{c}
\mathcal{Z}_{i}(1) \\
\frac{\Phi^{\prime}(\tau) \tau}{2}\left(\mathcal{Z}_{i}(2)+\mathcal{Z}_{i}(3)\right)
\end{array}\right)+\left(\begin{array}{c}
0 \\
\frac{\Phi^{\prime}(\tau) \tau}{2} \widetilde{\mathcal{Z}}
\end{array}\right)
$$

where, with $\mathbb{I}_{n} \equiv\left\{i=(j-1) k_{n}+1, \ldots,(j-1) k_{n}+m_{n} ; j=1, \ldots,\left\lfloor n / k_{n}\right\rfloor\right\}$, the elements of $\mathcal{Z}_{i}$ are defined as

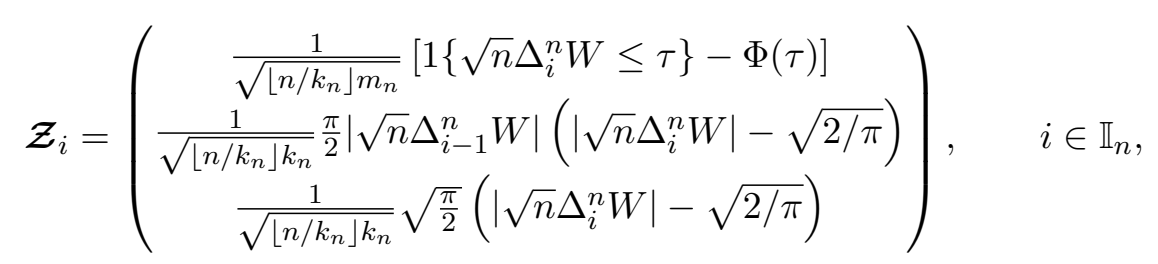

and, for $i=1, \ldots, n \backslash \mathbb{I}_{n}, \mathcal{Z}_{i}$ is defined as above, but with the first element replaced by zero. Finally, the residual term, $\widetilde{\mathcal{Z}}$, is defined with $\Delta_{0}^{n} W=0$ as

$$
\begin{aligned}
\widetilde{\mathcal{Z}} & =\frac{-(\pi / 2)}{\sqrt{\left\lfloor n / k_{n}\right\rfloor k_{n}}} \sum_{j=1}^{\left\lfloor n / k_{n}\right\rfloor} \widetilde{\mathcal{Z}}_{j}, \quad \text { where } \\
\widetilde{\mathcal{Z}}_{j} & =\left[\left|\sqrt{n} \Delta_{(j-1) k_{n}}^{n} W\right|\left(\left|\sqrt{n} \Delta_{(j-1) k_{n}+1}^{n} W\right|-\sqrt{\frac{2}{\pi}}\right)+\sqrt{\frac{2}{\pi}}\left(\left|\sqrt{n} \Delta_{j k_{n}}^{n} W\right|-\sqrt{\frac{2}{\pi}}\right)\right] .
\end{aligned}
$$

Then, locally uniformly in $\tau$ over compact subsets of $\mathbb{R}$, it follows that

(a) $\mathbb{E}_{i-1}^{n}\left[\mathcal{Z}_{i}\right]=\mathbf{0}, \sum_{i=1}^{\left\lfloor n / k_{n}\right\rfloor k_{n}} \mathbb{E}_{i-1}^{n}\left[\left\|\mathcal{Z}_{i}\right\|^{2+\iota}\right] \rightarrow 0, \forall \iota>0$ and

$$
\sum_{i=1}^{\left\lfloor n / k_{n}\right\rfloor k_{n}} \mathbb{E}_{i-1}^{n}\left[\mathcal{Z}_{i} \mathcal{Z}_{i}^{\prime}\right] \rightarrow \mathcal{C}_{\mathcal{Z}}(\tau), \quad \mathcal{C}_{\mathcal{Z}}(\tau) \equiv\left(\begin{array}{ccc}
\Phi(\tau)(1-\Phi(\tau)) & 0 & 0 \\
0 & (\pi / 2)^{2}(1-2 \pi) & (\pi / 2)(1-2 \pi) \\
0 & (\pi / 2)(1-2 \pi) & (\pi / 2)(1-2 \pi)
\end{array}\right)
$$

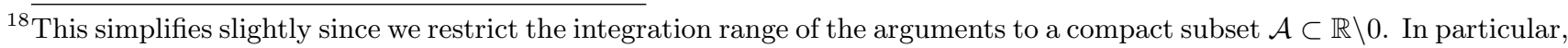
conditions (d) of both Lemmas 3.4 and 3.6 requires the integral to be well-behaved as the arguments $\tau_{1}, \tau_{2} \rightarrow \pm \infty$. Hence, as the integration is carried out over $\tau_{1}, \tau_{2} \in \mathcal{A}$, such conditions are avoided here.
} 
(b) Let $H_{1}(\tau)$ and $H_{2}(\tau)$ be defined as in Lemma 2, then $\mathbb{E}\left[\widetilde{\mathcal{Z}}^{2}\right] \leq K / k_{n}$ and

$$
\sum_{i=1}^{\left\lfloor n / k_{n}\right\rfloor k_{n}}\left(\begin{array}{c}
\mathcal{Z}_{i}(1) \\
\frac{\Phi^{\prime}(\tau) \tau}{2}\left(\mathcal{Z}_{i}(2)+\mathcal{Z}_{i}(3)\right)
\end{array}\right) \stackrel{d}{\rightarrow}\left(\begin{array}{l}
H_{1}(\tau) \\
H_{2}(\tau)
\end{array}\right) .
$$

Proof. This follows by the arguments on Todorov \& Tauchen (2014, pp. 1883-1884).

Lemma A.2 (Block Moments and CLT). For $i=1 \ldots,\left\lfloor n / k_{n}\right\rfloor k_{n}$, we let $\bar{v}_{i}^{*}=v_{(j-1) k_{n}+1}^{*}$ when $i \in(j-1) k_{n}+1, \ldots, j k_{n}$ with $j \in 1, \ldots,\left\lfloor n / k_{n}\right\rfloor$, and write $\mathcal{Z}_{i}^{*}(1)=\mathcal{Z}_{i}(1) v_{i}^{*}, \mathcal{Z}_{i}^{*}(2)=\mathcal{Z}_{i}(2) \bar{v}_{i}^{*}$ and $\mathcal{Z}_{i}^{*}(3)=\mathcal{Z}_{i}(3) \bar{v}_{i}^{*}$, and for which the triplet $\mathcal{Z}_{i}(1), \mathcal{Z}_{i}(2)$ and $\mathcal{Z}_{i}(3)$ are defined as in $(\mathrm{A} .21)$. Moreover, these are collected in the vector $\mathcal{Z}_{i}^{*}=\left(\mathcal{Z}_{i}^{*}(1), \mathcal{Z}_{i}^{*}(2), \mathcal{Z}_{i}^{*}(3)\right)^{\prime}$. Finally, let $K_{n}$ be a sequence of integers that satisfies $1 / K_{n}+K_{n} / n \rightarrow 0$ and $k_{n} / K_{n} \rightarrow \varrho_{k} \geq 0$ as $n \rightarrow \infty$, then

(a) $\frac{\left\lfloor n / k_{n}\right\rfloor k_{n}}{K_{n}} \sum_{i=1}^{K_{n}} \mathbb{E}_{i-1}^{n}\left[\mathcal{Z}_{i} \mathcal{Z}_{i}^{\prime}\right] \rightarrow \mathcal{C}_{\mathcal{Z}}(\tau)$ and, for $i \neq j, \frac{\left\lfloor n / k_{n}\right\rfloor k_{n}}{K_{n}} \sum_{i, j=1}^{K_{n}} \mathcal{Z}_{i} \mathcal{Z}_{j}^{\prime}=o_{p}(1)$.

(b) $\frac{\left\lfloor n / k_{n}\right\rfloor k_{n}}{K_{n}} \sum_{i=1}^{K_{n}} \mathbb{E}^{*}\left[\mathcal{Z}_{i}^{*}\left(\mathcal{Z}_{i}^{*}\right)^{\prime}\right] \stackrel{\mathbb{P}}{\rightarrow} \mathcal{C}_{\mathcal{Z}}(\tau)$ and, for $i \neq j, \frac{\left\lfloor n / k_{n}\right\rfloor k_{n}}{K_{n}} \sum_{i, j=1}^{K_{n}} \mathbb{E}^{*}\left[\mathcal{Z}_{i}^{*}\left(\mathcal{Z}_{j}^{*}\right)^{\prime}\right]=o_{p}^{*}(1)$.

(c) Locally uniformly in $\tau$ over compact subsets of $\mathbb{R}$,

$$
\sqrt{\frac{\left\lfloor n / k_{n}\right\rfloor k_{n}}{K_{n}}} \sum_{i=1}^{K_{n}} \mathcal{Z}_{i}^{*} \stackrel{d}{\rightarrow} N\left(\mathbf{0}, \mathcal{C}_{\mathcal{Z}}(\tau)\right) .
$$

Proof. The first part of (a) follows by changing the scale of $\mathcal{Z}_{i}$ and using Lemma A.1(a). The second part follows by using the Markov inequality for the martingale difference sequence, $\mathcal{Z}_{i}, i=1, \ldots, n$, and subsequently the $(2+\iota)$-moment result in Lemma A.1(a).

Next, for (b), utilize the decomposition $\mathbb{E}^{*}\left[\mathcal{Z}_{i}^{*}\left(\mathcal{Z}_{j}^{*}\right)^{\prime}\right]=\mathcal{Z}_{i} \mathcal{Z}_{j}^{\prime} \operatorname{Cov}\left(v_{i}^{*}, v_{j}^{*}\right)$ for all $i, j=1, \ldots, K_{n}$, which, in conjunction with (a) and Assumption DWB, delivers the results.

Last, for (c), and similarly to Todorov \& Tauchen (2014, pp. 1883-1884), $\mathbb{E}_{i-1}^{n}\left(\mathbb{E}^{*}\left[\mathcal{Z}_{i}^{*}\right]\right)=0$,

$$
\begin{gathered}
\frac{\left\lfloor n / k_{n}\right\rfloor k_{n}}{K_{n}} \sum_{i=1}^{K_{n}} \mathbb{E}_{i-1}^{n}\left(\mathbb{E}^{*}\left[\mathcal{Z}_{i}^{*}\left(\mathcal{Z}_{i}^{*}\right)^{\prime}\right]\right) \rightarrow \mathcal{C}_{\mathcal{Z}}(\tau), \quad \text { and } \\
\left.\left(\frac{\left\lfloor n / k_{n}\right\rfloor k_{n}}{K_{n}}\right)^{2} \sum_{i=1}^{K_{n}} \mathbb{E}_{i-1}^{n}\left(\mathbb{E}^{*}\left[\left\|\mathcal{Z}_{i}^{*}\right\|^{4}\right]\right) \leq K\left(\frac{\left\lfloor n / k_{n}\right\rfloor k_{n}}{K_{n}}\right)^{2} \sum_{i=1}^{K_{n}} \mathbb{E}_{i-1}^{n}\left(\left\|\mathcal{Z}_{i}\right\|^{4}\right) \mathbb{E}^{*}\left[\left\|\overline{\boldsymbol{v}}_{i}^{*}\right\|^{4}\right]\right) \rightarrow 0,
\end{gathered}
$$

with $\overline{\boldsymbol{v}}_{i}^{*}=\left(v_{i}^{*}, \bar{v}_{i}^{*}, \bar{v}_{i}^{*}\right)^{\prime}$, using the same arguments as for (a) and (b), Lemma A.1(a) as well as Assumption DWB(a). Together independence of the Brownian increments and successive conditioning under the $\mathbb{P}^{*}$ and $\mathbb{P}$ measures, we may invoke the central limit theorem for martingale difference sequences, e.g., Hall \& Heyde (1980, Chapter 3), to establish the limit result point-wise in $\tau$. An application of Billingsley (1968, Theorem 12.3) delivers the locally uniform result. 
Lemma A.3 (DWB central limit theory). Under the conditions of Theorem 1, then, locally uniformly in $\tau$ over compact subsets of $\mathbb{R}$,

$$
\left(\begin{array}{c}
\sqrt{\left\lfloor n / k_{n}\right\rfloor m_{n}} \widehat{H}_{n, 1}^{*}(\tau) \\
\sqrt{\left\lfloor n / k_{n}\right\rfloor k_{n}} \widehat{H}_{n, 2}^{*}(\tau)
\end{array}\right) \stackrel{d^{*}}{\rightarrow}\left(\begin{array}{c}
H_{1}(\tau) \\
H_{2}(\tau)
\end{array}\right),
$$

in probability- $\mathbb{P}$, where $H_{1}(\tau)$ and $H_{2}(\tau)$ are defined as in Lemma 2.

Proof. First, make a decomposition similarly to (A.20),

$$
\left(\begin{array}{l}
\sqrt{\left\lfloor n / k_{n}\right\rfloor m_{n}} \widehat{H}_{n, 1}^{*}(\tau) \\
\sqrt{\left\lfloor n / k_{n}\right\rfloor k_{n}} \widehat{H}_{n, 2}^{*}(\tau)
\end{array}\right) \equiv \sum_{i=1}^{\left\lfloor n / k_{n}\right\rfloor k_{n}}\left(\begin{array}{c}
\mathcal{Z}_{i}^{*}(1) \\
\frac{\Phi^{\prime}(\tau) \tau}{2}\left(\mathcal{Z}_{i}^{*}(2)+\mathcal{Z}_{i}^{*}(3)\right)
\end{array}\right)+\left(\begin{array}{c}
0 \\
\frac{\Phi^{\prime}(\tau) \tau}{2} \widetilde{\mathcal{Z}}^{*}
\end{array}\right)
$$

where the vector $\mathcal{Z}_{i}^{*}=\left(\mathcal{Z}_{i}^{*}(1), \mathcal{Z}_{i}^{*}(2), \mathcal{Z}_{i}^{*}(3)\right)^{\prime}, i=1, \ldots, n$, are defined as in Lemma A.2 and

$$
\widetilde{\mathcal{Z}}^{*}=\frac{-(\pi / 2)}{\sqrt{\left\lfloor n / k_{n}\right\rfloor k_{n}}} \sum_{j=1}^{\left\lfloor n / k_{n}\right\rfloor} \widetilde{\mathcal{Z}}_{j} v_{(j-1) k_{n}+1}^{*}
$$

As the residual term has $\mathbb{E}\left(\mathbb{E}^{*}\left[\widetilde{\mathcal{Z}}^{2}\right]\right) \leq K / k_{n}$ by Lemma A.1(b) together with Assumption DWB, we may focus on the first right-hand-side term in (A.23). Here, since $v_{i}^{*}$ in $\mathcal{Z}_{i}^{*}(1)$ is $b_{n}$-dependent by Assumption DWB, and $\bar{v}_{i}^{*}$ in $\mathcal{Z}_{i}^{*}(2)$ and $\mathcal{Z}_{i}^{*}(3)$ is $k_{n}$-dependent, we can adopt a large-block-small-block argument in conjunction with a modified Cramér-Wold device to show

$$
\sum_{i=1}^{\left\lfloor n / k_{n}\right\rfloor k_{n}} \boldsymbol{\lambda}^{\prime} \mathcal{Z}_{i} \stackrel{d^{*}}{\rightarrow} \boldsymbol{\lambda}^{\prime} \mathcal{Z}_{\infty}, \quad \text { in probability }-\mathbb{P}
$$

where $\boldsymbol{\lambda}$ is contained in a countable dense subset of the unit circle $\mathcal{D}=\left\{\boldsymbol{\lambda}_{k}: k \in \mathbb{N}\right\}$, and the asymptotic distribution $\mathcal{Z}_{\infty} \sim N\left(\mathbf{0}, \mathcal{C}_{\mathcal{Z}}(\tau)\right)$ with $\mathcal{C}_{\mathcal{Z}}(\tau)$ defined as in Lemma A.1(a). ${ }^{19}$ Hence, define a sequence of integers $K_{n}$ such that $K_{n} \rightarrow \infty$ and $K_{n} / n \rightarrow 0$ as $n \rightarrow \infty$, capturing the "large" block size. Moreover, let $\ell_{n}=\left\lfloor n /\left(K_{n}+k_{n}\right)\right\rfloor \rightarrow \infty$ be the number of blocks, then we may define blocks:

$$
\begin{aligned}
& \mathcal{L}_{r}=\left\{i \in \mathbb{N}:(r-1)\left(K_{n}+k_{n}\right)+1 \leq i \leq r\left(K_{n}+k_{n}\right)-k_{n}\right\}, \quad r=1, \ldots, \ell_{n}, \quad \text { and } \\
& \mathcal{S}_{r}=\left\{i \in \mathbb{N}: r\left(K_{n}+k_{n}\right)-k_{n}+1 \leq i \leq r\left(K_{n}+k_{n}\right)\right\}, \quad r=1, \ldots, \ell_{n}-1,
\end{aligned}
$$

as well as $\mathcal{S}_{\ell_{n}}\left\{i \in \mathbb{N}: \ell_{n}\left(K_{n}+k_{n}\right)-k_{n}+1 \leq i \leq n\right\}$. Now, conditional on the sample path $\mathcal{X}_{n}$, we have that $\mathcal{U}_{r}^{*}=\sum_{i \in \mathcal{L}_{r}} \boldsymbol{\lambda}^{\prime} \mathcal{Z}_{i}$ and $\mathcal{V}_{r}^{*}=\sum_{i \in \mathcal{S}_{r}} \boldsymbol{\lambda}^{\prime} \mathcal{Z}_{i}$ are independent across $r=1, \ldots, \ell_{n}$ and $r=$ $1, \ldots, \ell_{n}-1$ for $\mathcal{U}_{r}^{*}$ and $\mathcal{V}_{r}^{*}$, respectively. The proof, thus, proceeds by showing existence of sequences $K_{n}$ and $\ell_{n}$ such that the following conditions hold:

(i) $\sum_{r=1}^{\ell_{n}} \mathcal{V}_{r}^{*}=o_{p}^{*}(1)$, in probability- $\mathbb{P}$,

\footnotetext{
${ }^{19}$ A similar strategy is adopted for the proof on Shao (2010, Theorem 3.1), albeit with subtle and important differences.
} 
(ii) $\mathbb{E}^{*}\left[\sum_{r=1}^{\ell_{n}} U_{r}^{*}\right]=0$ and $\mathbb{E}^{*}\left[\left(\sum_{r=1}^{\ell_{n}} U_{r}^{*}\right)^{2}\right] \stackrel{\mathbb{P}}{\rightarrow} \mathcal{C}_{\mathcal{Z}}(\tau)$.

(iii) $\mathcal{I}_{n}^{*}(\epsilon) \equiv \sum_{r=1}^{\ell_{n}} \mathbb{E}^{*}\left[\left(U_{r}^{*}\right)^{2} 1\left\{\left|U_{r}^{*}\right|>\epsilon\right\}\right] \stackrel{\mathbb{P}}{\rightarrow} 0$, for some $\epsilon>0$.

since, in conjunction with $\mathcal{U}_{r}^{*}, r=1, \ldots, \ell_{n}$, this suffices to show (A.24) point-wise in $\tau$ over compact subsets of $\mathbb{R}$. The stated central limit theorem in the lemma for locally uniform intervals of $\tau$, then, follows by the Cramér-Wold theorem in conjunction with Billingsley (1968, Theorem 12.3).

First, for (i), we have $\mathbb{E}^{*}\left[V_{r}^{*}\right]=0$ by Assumption DWB. Moreover, for $r=1, \ldots, \ell_{n}-1$, it follows that $\mathbb{E}^{*}\left[\left(V_{r}^{*}\right)^{2}\right]=\sum_{i, j \in \mathcal{S}_{r}} \boldsymbol{\lambda}^{\prime} \mathcal{Z}_{i} \mathcal{Z}_{i}^{\prime} \boldsymbol{\lambda} \operatorname{Cov}\left(v_{i}^{*}, v_{j}^{*}\right)=O_{p}\left(k_{n} / n\right)$ by Lemma A.2(a) and $\operatorname{Cov}\left(v_{i}^{*}, v_{j}^{*}\right) \rightarrow$ $C_{i, j} \geq 0$ by Assumption DWB. By the same argument, we have $\mathbb{E}^{*}\left[\left(V_{\ell_{n}}^{*}\right)^{2}\right]=O_{p}\left(K_{n} / n\right)$. Hence, utilizing independence between the blocks, $V_{r}^{*}$, this provides the bound

$$
\mathbb{E}^{*}\left[\left(\sum_{r=1}^{\ell_{n}} V_{r}^{*}\right)^{2}\right]=O_{p}\left(\frac{\ell_{n} k_{n}}{n}+\frac{K_{n}}{n}\right),
$$

for which $\left(\ell_{n} k_{n}\right) / n \asymp k_{n} / K_{n} \rightarrow 0$ and $K_{n} / n \rightarrow 0$ as $n \rightarrow \infty$, thereby showing (i).

Next, (ii) follows by Assumption DWB, Lemma A.2(b) and independence between the blocks in the sequence $U_{r}^{*}, r=1, \ldots, \ell_{n}$, under the bootstrap measure.

Last, for the Lindeberg condition in (iii), it suffices to show $\mathbb{E}\left[\mathcal{I}_{n}^{*}(\epsilon)\right] \rightarrow 0$. Now, by stationarity of the bootstrap variables and independence of the Brownian increments in $\mathcal{Z}_{i}$,

$$
\mathbb{E}\left[\mathcal{I}_{n}^{*}(\epsilon)\right] \leq K \ell_{n} \mathbb{E}\left(\mathbb{E}^{*}\left[\left(U_{1}^{*}\right)^{2} 1\left\{\left|U_{1}^{*}\right|>\epsilon\right\}\right]\right)=K \mathbb{E}\left(\mathbb{E}^{*}\left[\left(\sqrt{\ell_{n}} U_{1}^{*}\right)^{2} 1\left\{\left|\sqrt{\ell_{n}} U_{1}^{*}\right|>\sqrt{\ell_{n}} \epsilon\right\}\right]\right) .
$$

Hence, it suffices to analyze the properties of $\sqrt{\ell_{n}} U_{1}^{*}$ when expectations are taken under both random measures. Indeed, since $\ell_{n} /\left(\left\lfloor n / k_{n}\right\rfloor k_{n} / K_{n}\right) \rightarrow 1$ as $n \rightarrow \infty$, the use of the continuous mapping theorem and Slutsky's theorem in combination with Lemma A.2(b)-(c) establish that $\sqrt{\ell_{n}} U_{1}^{*} \stackrel{d}{\rightarrow} \boldsymbol{\lambda}^{\prime} \mathcal{Z}_{\infty}$ as well as $\mathbb{E}\left(\mathbb{E}^{*}\left[\ell_{n}\left(U_{1}^{*}\right)^{2}\right]\right) \rightarrow \boldsymbol{\lambda}^{\prime} \mathcal{C}_{\mathcal{Z}}(\tau) \boldsymbol{\lambda}$ as $n \rightarrow \infty$, locally uniformly in $\tau$ over compact subsets of $\mathbb{R}$. Hence, these results imply uniform integrability of $\left(\sqrt{\ell_{n}} U_{1}^{*}\right)^{2}$, providing

$$
\mathbb{E}\left(\mathbb{E}^{*}\left[\left(\sqrt{\ell_{n}} U_{1}^{*}\right)^{2} 1\left\{\left|\sqrt{\ell_{n}} U_{1}^{*}\right|>\sqrt{\ell_{n}} \epsilon\right\}\right]\right) \rightarrow \mathbb{E}\left(\mathbb{E}^{*}\left[\left(\boldsymbol{\lambda}^{\prime} \mathcal{Z}_{\infty}\right)^{2} 1\left\{\left|\boldsymbol{\lambda}^{\prime} \mathcal{Z}_{\infty}\right|>\sqrt{\ell_{n}} \epsilon\right\}\right]\right) \rightarrow 0
$$

since $\sqrt{\ell_{n}} \epsilon \rightarrow \infty$ when $n \rightarrow \infty$. This shows (iii), thereby concluding the proof.

\section{B Standard Local Gaussian Resampling}

To elaborate on Remark 3, we follow Hounyo (2018) and generate the high-frequency innovations as,

$$
\Delta_{(j-1) k_{n}+i}^{n} Z^{*}=\sqrt{\frac{\widehat{V}_{n, j}}{n}} u_{i+(j-1) k_{n}}^{*}, \quad i=1, \ldots, k_{n}, j=1, \ldots,\left\lfloor n / k_{n}\right\rfloor,
$$


where $u_{i+(j-1) k_{n}}^{*} \sim$ i.i.d. $N(0,1)$ across the $(i, j)$ indices. Using these, the analogous bootstrap spot variation estimator may be decomposed as $\widehat{V}_{n, j}^{*}=\widehat{V}_{n, j} U_{n, j}^{*}$, where

$$
\widehat{V}_{n, j}^{*}=\frac{\pi}{2} \frac{n}{k_{n}-1} \sum_{i=(j-1) k_{n}+2}^{j k_{n}}\left|\Delta_{i-1}^{n} Z^{*}\right|\left|\Delta_{i}^{n} Z^{*}\right|, \quad U_{n, j}^{*}=\frac{\pi}{2\left(k_{n}-1\right)} \sum_{i=(j-1) k_{n}+2}^{j k_{n}}\left|u_{i-1}^{*}\right|\left|u_{i}^{*}\right|,
$$

utilizing that $\widehat{V}_{n, j}$ is constant over $i$ for a given $j$. Moreover, by first defining $\widehat{V}_{n, j}^{*}(i)$ similarly to (6), that is, replacing $\Delta_{i}^{n} Z$ with $\Delta_{i}^{n} Z^{*}$, and using the definition of $\widehat{V}_{n, j}^{*}$, the former reduces to

$$
\widehat{V}_{n, j}^{*}(i)=\widehat{V}_{n, j} U_{n, i, j}^{*}(i),
$$

where, by expanding and rewriting the analogue of (6), we have

$$
U_{n, i, j}^{*}(i)=\left\{\begin{array}{c}
\frac{\pi}{2} \frac{1}{k_{n}-3}\left(\sum_{l=(j-1) k_{n}+2}^{j k_{n}}\left|u_{l-1}^{*}\right|\left|u_{l}^{*}\right|-\left|u_{i}^{*}\right|\left|u_{i+1}^{*}\right|\right) \quad \text { for } \quad i=(j-1) k_{n}+1 ; \\
\frac{\pi}{2} \frac{1}{k_{n}-3}\left(\sum_{l=(j-1) k_{n}+2}^{j k_{n}}\left|u_{l-1}^{*}\right|\left|u_{l}^{*}\right|-\left(\left|u_{i-1}^{*}\right|\left|u_{i}^{*}\right|+\left|u_{i}^{*}\right|\left|u_{i+1}^{*}\right|\right)\right), \\
\quad \text { or } i=(j-1) k_{n}+2, \ldots, j k_{n}-1 ; \\
\frac{\pi}{2} \frac{1}{k_{n}-3}\left(\sum_{l=(j-1) k_{n}+2}^{j k_{n}}\left|u_{l-1}^{*}\right|\left|u_{l}^{*}\right|-\left|u_{i-1}^{*}\right|\left|u_{i}^{*}\right|\right), \quad \text { for } i=j k_{n} .
\end{array}\right.
$$

Now, it is important to note that both bootstrap spot variation estimators, $\widehat{V}_{n, j}^{*}$ and $\widehat{V}_{n, j}^{*}(i)$, both decompose into $\widehat{V}_{n, j}$ as well as additional terms that consist exclusively of the resampled data. This implies that when forming the bootstrap empirical CDF, the key ratios reduce to

$$
\mathcal{R}_{n, i, j}^{*}=\frac{\sqrt{n} \Delta_{i}^{n} Z^{*}}{\sqrt{\widehat{V}_{n, j}^{*}}}=\frac{u_{i}^{*}}{\sqrt{U_{n, i, j}^{*}}}, \quad \mathcal{R}_{n, i, j}^{*}(i)=\frac{\sqrt{n} \Delta_{i}^{n} Z^{*}}{\sqrt{\widehat{V}_{n, j}^{*}(i)}}=\frac{u_{i}^{*}}{\sqrt{U_{n, i, j}^{*}(i)}}
$$

with $i=(j-1) k_{n}+1, \ldots,(j-1) k_{n}+m_{n}$. In other words, $\mathcal{R}_{n, i, j}^{*}$ and $\mathcal{R}_{n, i, j}^{*}(i)$ no longer depend on the original data. However, since the two ratios preserve the exact dependence structure of the corresponding ratios in empirical CDF, $\widehat{F}_{n}(\tau)$, the relations in (B.4) can be used to simulate the asymptotic distribution of $\widehat{F}_{n}(\tau)$ under the null hypothesis $\mathcal{H}_{0}: S_{t}=W_{t}$, which may generate improvements of the finite sample inference. Hence, if one considers the resampled empirical CDF,

$$
\widehat{F}_{\mathcal{R}, n}^{*}(\tau)=\frac{1}{N_{\mathcal{R}, n}^{*}(\alpha, \varpi)} \sum_{j=1}^{\left\lfloor n / k_{n}\right\rfloor} \sum_{i=(j-1) k_{n}+1}^{(j-1) k_{n}+m_{n}} 1\left\{\mathcal{R}_{n, i, j}^{*}(i) \leq \tau\right\} 1\left\{\left|\mathcal{R}_{n, i, j}^{*}\right| \leq \alpha n^{1 / 2-\varpi}\right\},
$$

where

$$
N_{\mathcal{R}, n}^{*}(\alpha, \varpi)=\sum_{j=1}^{\left\lfloor n / k_{n}\right\rfloor} \sum_{i=(j-1) k_{n}+1}^{(j-1) k_{n}+m_{n}} 1\left\{\left|\mathcal{R}_{n, i, j}^{*}\right| \leq \alpha n^{1 / 2-\varpi}\right\},
$$


and we redefine $\tilde{u}_{i}^{*}=u_{i}^{*} / \sqrt{n} \stackrel{d}{=} N\left(0, \Delta_{i}^{n}\right)$, then this process (and CDF statistic) belong to the general class (3) as a special case with $\sigma_{t}=1, \alpha_{t}=0, Y_{t}=0$ and $S_{t}=W_{t}$ for all $0 \leq t \leq 1$. Hence, the CLT for the Gaussian resampled $\mathrm{CDF}, \widehat{F}_{\mathcal{R}, n}^{*}(\tau)$, may be obtained as a corollary to Lemma 2 :

Corollary 4. Suppose that (B.1) holds, then, locally uniformly in $\tau$ over any compact subset $\mathcal{A} \subset$ $\mathbb{R} \neq 0$,

$$
\widehat{F}_{\mathcal{R}, n}^{*}(\tau)-\Phi(\tau)=\widehat{H}_{\mathcal{R}, n, 1}^{*}(\tau)+\widehat{H}_{\mathcal{R}, n, 2}^{*}(\tau)+H_{3}(\tau) / k_{n}+o_{p}\left(1 / k_{n}\right)
$$

where $\sqrt{\left\lfloor n / k_{n}\right\rfloor m_{n}}\left(\widehat{H}_{\mathcal{R}, n, 1}^{*}(\tau), \sqrt{k_{n} / m_{n}} \widehat{H}_{\mathcal{R}, n, 2}^{*}(\tau)\right) \stackrel{d}{\rightarrow}\left(H_{\mathcal{R}, 1}^{*}(\tau), H_{\mathcal{R}, 2}^{*}\right)$ with $H_{\mathcal{R}, 1}^{*}$ and $H_{\mathcal{R}, 2}^{*}(\tau)$ being two independent Gaussian processes with covariances similar to those for $H_{1}(\tau)$ and $H_{2}(\tau)$, respectively, in (9). Finally, $H_{3}(\tau)$ is defined in (10).

Since the local Gaussian CDF statistic, $\widehat{F}_{\mathcal{R}, n}^{*}(\tau)$, is a special case of the empirical CDF without impact from drift, residual jumps, and stochastic volatility, while exactly capturing its dependence structure, one could base inference for $\widehat{F}_{n}(\tau)$ and its Kolmogorov-Smirnov test, $T_{n}$, on the resample distributions $\widehat{F}_{\mathcal{R}, n}^{*}(\tau)-\Phi(\tau)$ and $\sup _{\tau \in \mathcal{A}} \sqrt{N_{\mathcal{R}, n}^{*}(\alpha, \varpi)}\left|\widehat{F}_{\mathcal{R}, n}^{*}(\tau)-\Phi(\tau)\right|$. However, as this inference procedure has lost all dependence on the original data, it likely suffers from finite sample distortions similarly to those affecting the asymptotic distribution when the underlying process indeed exhibits drift, jumps and stochastic volatility. Hence, we prefer, and recommend, the use of the LDWB inference procedure in Section 3.2, which not only preserves dependence on the original data, it also replicates the second-order asymptotic theory induced by the nonparametric standardization.

\section{Implementation Details}

In this section, we detail how one can implement the proposed bootstrap tests. Let $B$ denote the number of bootstrap replications for each of the $M$ Monte Carlo replications. Then, for a given equidistant partition of the normalized time window $[0,1]$ with step length $1 / n$ do the following:

\section{Algorithm 1: The LDWB and/or the NLBB procedure for hypothesis testing}

Step 1. Simulate $n+1 \in \mathbb{N}$ points of the process $Z_{t}$ under investigation (a pure-jump semimartingale or a jump diffusion, or a jump diffusion contaminated by noise).

Step 2. Compute $n$ intraday returns at an equidistant time grid $t_{i} \equiv i / n \in[0,1]$, for $i=0, \ldots, n$, as the innovation $\Delta_{i}^{n} Z=Z_{t_{i}}-Z_{t_{i-1}}$.

Step 3. Compute the Kolmogorov-Smirnov statistic,

$$
\widehat{\mathrm{KS}}_{n}(\mathcal{A})=\sup _{\tau \in \mathcal{A}} \sqrt{N_{n}(\alpha, \varpi)}\left|\widehat{F}_{n}(\tau)-\Phi(\tau)\right|,
$$


where $N_{n}(\alpha, \varpi)$ and $\widehat{F}_{n}(\tau)$ are defined as in (7) and (8), respectively. For the compact set $\mathcal{A}$, one may, e.g., choose (as in Section 5),

$$
\mathcal{A}=[Q(0.001): Q(0.499)] \cup[Q(0.501): Q(0.999)]
$$

where $Q(\theta)$ is the $\theta$-quantile of the standard normal distribution.

Step 4. Generate an $m_{n}\left\lfloor n / k_{n}\right\rfloor$ sequence of external random random variables $v_{(j-1) k_{n}+i}^{*}$, for running indices $i=1, \ldots, m_{n}, j=1, \ldots,\left\lfloor n / k_{n}\right\rfloor$, which are independent of the observations generated in Step 1 as well as satisfy the conditions of Assumption DWB. As advocated in Section 5.1, one may use the random variables underlying DWB1, DWB2, DWB3 or DWB4. ${ }^{20}$

Step 5. Generate the locally dependent wild bootstrap observations as in (14).

Step 6. Compute the bootstrap Kolmogorov-Smirnov statistic $\operatorname{KS}_{n}^{*}(\mathcal{A})$ as in $(21)$. In particular,

$$
\mathrm{KS}_{n}^{*}(\mathcal{A})=\sup _{\tau \in \mathcal{A}} \sqrt{N_{n}(\alpha, \varpi)}\left|\widehat{F}_{W, n}^{*}(\tau)-\widehat{F}_{n}(\tau)\right|
$$

where $N_{n}(\alpha, \varpi), \widehat{F}_{W, n}^{*}(\tau), \widehat{F}_{n}(\tau)$ and $\mathcal{A}$ are defined as in (7), (15), (8) and (C.1), respectively.

Step 7. Repeat Steps 4-6 $B$ times and keep the values of $\operatorname{KS}_{n}^{*(j)}(\mathcal{A}), j=1, \ldots, B$, where $\operatorname{KS}_{n}^{*(j)}(\mathcal{A})$ is given as in Step 6. Then, sort $\operatorname{KS}_{n}^{*(1)}(\mathcal{A}), \ldots, \mathrm{KS}_{n}^{*(B)}(\mathcal{A})$ ascendingly from the smallest to the largest as $\overline{\mathrm{KS}}_{n}^{*(1)}(\mathcal{A}), \ldots, \overline{\mathrm{KS}}_{n}^{*(B)}(\mathcal{A})$ such that $\overline{\mathrm{KS}}_{n}^{*(i)}(\mathcal{A})<\overline{\mathrm{KS}}_{n}^{*(j)}(\mathcal{A})$ for all $1 \leq i<j \leq B$.

Step 8. Reject $\mathcal{H}_{0}: S_{t}=W_{t}$ when $\widehat{\mathrm{KS}}_{n}(\mathcal{A})>q_{n}^{*}(\alpha, \mathcal{A})$ where $q_{n}^{*}(\alpha, \mathcal{A})$ is the $\alpha$ quantile of the bootstrap distribution of $\operatorname{KS}_{n}^{*}(\mathcal{A})$. For example, if we let $B=999$, then the 0.05-th quantile of $\mathrm{KS}_{n}^{*}(\mathcal{A})$ is estimated by $\mathrm{KS}_{n}^{*(a)}(\mathcal{A})$ with $a=0.05 \times(999+1)=50$.

Step 9. Repeat Steps 1-8 $M$ times to get the size or power of the bootstrap test. In particular, if $Z_{t}$ is simulated as a jump diffusion, then the size is given by $M^{-1}\left(\#\left\{\widehat{\operatorname{KS}}_{n}(\mathcal{A})>q_{n}^{*}(\alpha, \mathcal{A})\right\}\right)$.

\footnotetext{
${ }^{20}$ For the NLBB, note that observations can be obtained equivalently by resampling, as in equation (17), or by generating external random variable as follows: $v_{(j-1) k_{n}+i}^{*}=\zeta_{p_{n}, j}^{*}-1$ for $i=1, \ldots, m_{n}$ across blocks $j=1, \ldots,\left\lfloor n / k_{n}\right\rfloor$, where we let $p_{n}=\left\lfloor n / k_{n}\right\rfloor$ and $\zeta_{p_{n}, j}^{*}, j=1, \ldots, p_{n}$ be a sequence of multinomial random variables with probability $1 / p_{n}$ and number of trials $p_{n}$, see Section 3.3 for further details.
} 


\section{References}

Ait-Sahalia, Y. \& Jacod, J. (2009), 'Estimating the degree of activity of jumps in high frequency data', Annals of Statistics 37, 2202-2244.

Aït-Sahalia, Y. \& Jacod, J. (2010), 'Is Brownian motion necessary to model high-frequency data?', Annals of Statistics 38, 3093-3128.

Andersen, T. G. \& Benzoni, L. (2012), Stochastic volatility, in R. A. Meyers, ed., 'Encyclopedia of Complexity and Systems Science', Springer-Verlag. forthcoming.

Andersen, T. G., Bollerslev, T. \& Dobrev, D. (2007), 'No-arbitrage semi-martingale restrictions for continuoustime volatility models subject to leverage effects, jumps and i.i.d. noise: Theory and testable distributional implications', Journal of Econometrics 138, 125-180.

Andersen, T. G., Bondarenko, O., Todorov, V. \& Tauchen, G. (2015), 'The fine structure of equity-index option dynamics', Journal of Econometrics 187, 532-546.

Andersen, T. G., Fusari, N., Todorov, V. \& Varneskov, R. T. (2018), Option panels in pure-jump settings. Unpublished manuscript, Northwestern University.

Ané, T. \& Geman, H. (2000), 'Order flow, transaction clock and normality of asset returns', Journal of Finance 55, 2259-2284.

Arcones, M. A. \& Giné, E. (1992), 'On the bootstrap of $U$ and $V$ statistics', Annals of Statistics 20, 655-674.

Back, K. (1991), 'Asset prices for general processes', Journal of Mathematical Economics 20, 317-395.

Baeumer, B. \& Meerschaert, M. (2009), 'Tempered stable levy motion and transit superdiffusion', Journal of Computational and Applied Mathematics 223, 2438-2448.

Bandi, F. M. \& Russell, J. R. (2008), 'Microstructure noise, realized variance, and optimal sampling', Review of Economic Studies 75, 339-369.

Barndorff-Nielsen, O. E. \& Shephard, N. (2001), 'Non-gaussian Ornstein-Uhlenbeck-based models and some of their uses in financial economics', Journal of the Royal Statistical Society Series B 63, 167-241.

Beutner, E. \& Zähle, H. (2014), 'Continuous mapping approach to the asymptotics of $U$ - and $V$-statistics', Bernoulli 20, 846-877.

Bhattacharya, R. N. \& Rao, R. R. (1986), Normal Approximation and Asymptotic Expansions, Siam: Classics in Applied Mathematics.

Bickel, P. J. \& Freedman, D. A. (1981), 'Some asymptotic theory for the bootstrap', Annals of Statistics 9, 1196-1217.

Billingsley, P. (1968), Convergence of Probability Measures, Wiley, New York.

Bontemps, C. \& Meddahi, N. (2005), 'Testing normality: A GMM approach', Journal of Econometrics 124, 149186.

Bühlmann, P. (1994), 'Blockwise bootstrapped empirical processes for stationary sequences', Annals of Statistics 22, 995-1012. 
Carlstein, E. (1986), 'The use of subseries values for estimating the variance of a general statistic from a stationary time series', Annals of Statistics 14, 1171-1179.

Carr, P., Geman, H., Madan, D. B. \& Yor, M. (2002), 'The fine structure of asset returns: An empirical investigation', Journal of Business 75, 305-332.

Carr, P., Geman, H., Madan, D. B. \& Yor, M. (2003), 'Stochastic volatility for Lévy processes', Mathematical Finance 58, 345-382.

Carr, P. \& Wu, L. (2003), 'The finite moment log stable process and option pricing', The Journal of Finance LVIII, 753-778.

Carr, P. \& Wu, L. (2004), 'Time-changed lévy processes and option pricing', Journal of Financial Economics 17, 113-141.

Chernov, M., Gallant, A. R., Ghysels, E. \& Tauchen, G. (2003), 'Alternative models for stock price dynamics', Journal of Econometrics 116, 225-257.

Clark, P. K. (1973), 'A subordinated stochastic process model with finite variance for speculative prices', Econometrica 41, 135-155.

Cont, R. \& Mancini, C. (2011), 'Nonparametric tests for pathwise properties of semimartingales', Bernoulli 17, 781-813.

Davidson, R. \& Flachaire, E. (2008), 'The wild bootstrap, tamed at last', Journal of Econometrics 146, 162-169.

Davidson, R. \& MacKinnon, J. G. (2006), 'The power of bootstrap and asymptotic tests', Journal of Econometrics 133, 421-441.

Delbaen, F. \& Schachermayer, W. (1994), 'A general version of the fundamental version of asset pricing', Mathematische Annalen 300, 463-520.

Doukhan, P., Lang, G., Leucht, A. \& Neumann, M. H. (2015), 'Dependent wild bootstrap for the empirical process', Journal of Time Series Analysis 36, 290-314.

Dovonon, P., Gonçalves, S., Hounyo, U. \& Meddahi, N. (2018), 'Bootstrapping high-frequency jump tests', Journal of the American Statistical Association forthcoming.

Efron, B. (1979), 'Bootstrap methods: Another look at the jackknife', Annals of Statistics 7, 1-26.

Fama, E. (1963), 'Mandelbrot and the stable paretian hypothesis', Journal of Business 36, 420-429.

Fama, E. \& Roll, R. (1968), 'Some properties of symmetric stable processes', Journal of the American Statistical Association 63, 817-836.

Gonçalves, S. \& Meddahi, N. (2009), 'Bootstrapping realized volatility', Econometrica 1, 283-306.

Hall, P. \& Heyde, C. C. (1980), Martingale Limit Theory and Its Appplication, Boston: Academic Press.

Hansen, P. R. \& Lunde, A. (2006), 'Realized variance and market microstructure noise', Journal of Business and Economic Statistics 24, 127-161.

Horowitz, J. L. \& Savin, N. E. (2000), 'Empirically relevant critical values for hypothesis tests: A bootstrap approach', Journal of Econometrics 95, 375-389. 
Hounyo, U. (2018), 'A local gaussian bootstrap method for realized volatility and realized beta', Econometric Theory forthcoming.

Hounyo, U. \& Varneskov, R. T. (2017), 'A local stable bootstrap for power variations of pure-jump semimartingales and activity index estimation', Journal of Econometrics 198, 10-28.

Huang, X. \& Tauchen, G. (2005), 'The relative contribution of jumps to total price variance', Journal of Financial Econometrics 3, 456-499.

Jacod, J. (2012), Statistics and high frequency data, in M. Kessler, A. Lindner \& M. Sørensen, eds, 'Statistical Methods for Stochastic Differential Equations', CRC Press.

Jacod, J. \& Protter, P. (2012), Discretization of Processes, Springer-Verlag: Berlin.

Jing, B.-Y., Kong, X.-B. \& Liu, Z. (2012), 'Modeling high-frequency financial data by pure jump processes', The Annals of Statistics 40, 759-784.

Kelly, B. \& Jiang, H. (2014), 'Tail risk and asset prices', Review of Financial Studies 27, 817-836.

Klüppelberg, C., Meyer-Brandi, T. \& Schmidt, A. (2010), 'Electricity spot price modelling with a view towards extreme spike risk', Quantitative Finance 10, 963-974.

Kunsch, H. R. (1989), 'The jackknife and the bootstrap for general stationary observations', Annals of Statistics 17, $1217-1241$.

Leucht, A. \& Neumann, M. H. (2013), 'Dependent wild bootstrap for degenerate $U$ - and $V$-statistics', Journal of Multivariate Analysis 117, 257-280.

Liu, R. Y. (1988), 'Bootstrap procedures under some non-i.i.d. models', Annals of Statistics 16, 1696-1708.

Liu, R. Y. \& Singh, K. (1992), Moving blocks jackknife and bootstrap capture weak dependence, in R. LePage \& L. Bilard, eds, 'Exploring the Limits of Bootstrap', New York: Wiley, pp. 225-248.

Mandelbrot, B. (1961), 'Stable paretian random functions and the multiplicative variation of income', Econometrica 29, 517-543.

Mandelbrot, B. (1963), 'The variation of certain speculative prices', Journal of Business 36, 394-419.

Mikosch, T., Resnik, S., Rootzen, H. \& Stegeman, A. (2002), 'Is network traffic approximated approximated by stable Levy montion or fractional Brownian motion?', Annals of Applied Probability 12, 23-68.

Monroe, I. (1978), 'Processes that can be embedded in Brownian motion', Annals of Probability 6, 42-56.

Naik-Nimbalkar, U. V. \& Rajarshi, M. B. (1994), 'Validity of blockwise bootstrap for empirical processes with stationary observations', Annals of Statistics 22, 980-994.

Paparoditis, E. \& Politis, D. N. (2001), 'Tapered block bootstrap', Biometrika 88, 1105-1119.

Paparoditis, E. \& Politis, D. N. (2002), 'The tapered block bootstrap for general statistics from stationary sequences', Econometrics Journal 5, 131-148.

Præstgaard, J. \& Wellner, J. A. (1993), 'Exchangeability weighted bootstraps of the general empirical process', Annals of Probability 21, 2053-2086.

Sato, K. (1999), Lévy processes and Infinitely Divisible Distributions, Cambridge University Press, United Kingdom. 
Shao, X. (2010), 'The dependent wild bootstrap', Journal of the American Statistical Association 105, 218-235.

Shao, X. (2011), 'A bootstrap-assisted spectral test of white noise under unknown dependence', Journal of Econometrics 162, 213-224.

Shapiro, S. S. \& Wilk, M. B. (1965), 'An analysis of variance test for normality (complete samples)', Biometrika 52, 591-611.

Shapiro, S. S., Wilk, M. B. \& Chen, H. J. (1968), 'A comparative study of various tests for normality', Journal of the American Statistical Association 63, 1343-1372.

Smeekes, S. \& Urbain, J.-P. (2014), A multivariate invariance principle for modified wild bootstrap methods with an application to unit root testing. Unpublished manuscript, Maastricht University.

Stephens, M. A. (1974), 'EDF statistics for goodness of fit and some comparisons', Journal of the American Statistical Association 69, 730-737.

Todorov, V. (2009), 'Estimation of continuous-time stochastic volatility models with jumps using high-frequency data', Journal of Econometrics 148, 131-148.

Todorov, V. (2015), 'Jump activity estimation for pure-jump semimartingales via self-normalized statistics', Annals of Statistics 43, 1831-1864.

Todorov, V. \& Tauchen, G. (2010), 'Activity signature functions for high-frequency data analysis', Journal of Econometrics 154, 125-138.

Todorov, V. \& Tauchen, G. (2011a), 'Limit theorems for power variations of pure-jump processes with application to activity estimation', The Annals of Applied Probability 21, 546-588.

Todorov, V. \& Tauchen, G. (2011b), 'Volatility jumps', Journal of Business and Economic Statistics 219, 356371.

Todorov, V. \& Tauchen, G. (2012), 'Realized laplace transforms for pure-jump semimartingales', Annals of Statistics 40, 1233-1262.

Todorov, V. \& Tauchen, G. (2014), 'Limit theorems for the empirical distribution function of scaled increments of Itô semimartingales at high frequencies', Annals of Applied Probability 24, 1850-1888.

Todorov, V., Tauchen, G. \& Grynkiv, I. (2014), 'Volatility activity: Specification and estimation', Journal of Econometrics 178, 180-193.

van der Vaart, A. W. (1998), Asymptotic Statistics, Cambridge University Press, Cambridge.

Wu, L. (2008), Modeling financial security returns using Lévy processes, in J. Birge \& L. Linetsky, eds, 'Handbooks in Operations Research and Management Science, Volume 15: Financial Engineering', Elsevier, North Holland. 
2017-38: Nektarios Aslanidis and Charlotte Christiansen: Flight to Safety from European Stock Markets

2017-39: Tommaso Proietti, Niels Haldrup and Oskar Knapik: Spikes and memory in (Nord Pool) electricity price spot prices

2018-01: Emilio Zanetti Chini: Forecaster's utility and forecasts coherence

2018-02: Torben G. Andersen, Nicola Fusari and Viktor Todorov: The Pricing of Tail Risk and the Equity Premium: Evidence from International Option Markets

2018-03: Torben G. Andersen, Nicola Fusari, Viktor Todorov and Rasmus T. Varneskov: Unified Inference for Nonlinear Factor Models from Panels with Fixed and Large Time Span

2018-04: Torben G. Andersen, Nicola Fusari, Viktor Todorov and Rasmus T. Varneskov: Option Panels in Pure-Jump Settings

2018-05: $\quad$ Torben G. Andersen, Martin Thyrsgaard and Viktor Todorov: Time-Varying Periodicity in Intraday Volatility

2018-06: $\quad$ Niels Haldrup and Carsten P. T. Rosenskjold: A Parametric Factor Model of the Term Structure of Mortality

2018-07: Torben G. Andersen, Nicola Fusari and Viktor Todorov: The Risk Premia Embedded in Index Options

2018-08: $\quad$ Torben G. Andersen, Nicola Fusari and Viktor Todorov: Short-Term Market Risks Implied by Weekly Options

2018-09: $\quad$ Torben G. Andersen and Rasmus T. Varneskov: Consistent Inference for Predictive Regressions in Persistent VAR Economies

2018-10: Isabel Casas, Xiuping Mao and Helena Veiga: Reexamining financial and economic predictability with new estimators of realized variance and variance risk premium

2018-11: $\quad$ Yunus Emre Ergemen and Carlos Velasco: Persistence Heterogeneity Testing in Panels with Interactive Fixed Effects

2018-12: $\quad$ Hossein Asgharian, Charlotte Christiansen and Ai Jun Hou: Economic Policy Uncertainty and Long-Run Stock Market Volatility and Correlation

2018-13: Emilio Zanetti Chini: Forecasting dynamically asymmetric fluctuations of the U.S. business cycle

2018-14: Cristina Amado, Annastiina Silvennoinen and Timo Teräsvirta: Models with Multiplicative Decomposition of Conditional Variances and Correlations

2018-15: Changli He, Jian Kang, Timo Teräsvirta and Shuhua Zhang: The Shifting Seasonal Mean Autoregressive Model and Seasonality in the Central England Monthly Temperature Series, 1772-2016

2018-16: $\quad$ Ulrich Hounyo and Rasmus T. Varneskov: Inference for Local Distributions at High Sampling Frequencies: A Bootstrap Approach 